\title{
Correlation Tests and Linear Spectral Statistics of the Sample Correlation Matrix
}

\author{
Xavier Mestre, Pascal Vallet
}

\begin{abstract}
Testing the independence of the entries of multidimensional Gaussian observations is a very important problem in statistics, with a number of applications in signal processing, radar, cognitive radio, seismography and multiple other fields. Typically, the problem is formulated as a binary hypothesis test, whereby the presence of correlation is declared when the value of a certain statistic is higher than a certain predetermined threshold. Most of the statistics for correlation tests are constructed from the sample correlation matrix (also known as sample coherence matrix in signal processing), which is defined as a power-normalized version of the sample covariance matrix. In this paper, correlation tests constructed from linear spectral statistics (LSS) of the sample correlation matrix are analyzed under the asymptotic framework where both sample size and observation dimension become large but comparable in magnitude. A Central Limit Theorem (CLT) is established on this class of statistics, which is valid for generally correlated Gaussian observations. Results show that LSS asymptotically fluctuate as Gaussian random variables under both hypotheses, with an asymptotic mean and variance that can be established for each particular test. In particular, this general CLT can be used to establish the asymptotic behavior of two of the most important correlation test statistics, namely the Generalized Likelihood Ratio Test (GLRT) and the Frobenius Norm Test (FNT), under both null and alternative hypotheses. As a by-product, it is established that LSS of sample covariance and sample correlation matrices have exactly the same first order behavior, but quite different asymptotic fluctuations in the second order regime. In both cases, the LSS asymptotically behave as Gaussian random variables, although with quite different asymptotic means and variances.
\end{abstract}

Index Terms-Linear Spectral Statistics, Coherence Matrix, Hadamard test, Random Matrix Theory.

\section{INTRODUCTION}

The detection of correlation between multiple Gaussian random signals is an important problem in multiple scientific fields, such as wireless sensor networks, multiantenna radar, radioastronomy [1], [2], cognitive radio or cooperative communications. The problem is equivalent to testing whether the covariance matrix of the observations has a diagonal structure, and can typically be formulated as a binary hypothesis test.

In order to introduce the problem, let $\mathbf{y}_{n}, n=1, \ldots, N$, denote a collection of $M$-dimensional random observation vectors, which are assumed to be zero mean, independent and identically distributed (i.i.d.) according to an $M$-variate law with covariance matrix $\mathbf{R}_{M}$. The detection of correlation

$\mathrm{X}$. Mestre is with the Centre Tecnològic de Telecomunicacions de Catalunya (CTTC/CERCA) (xavier.mestre@cttc. cat). P. Vallet is with the Institut Polytechnique de Bordeaux (pascal.vallet@bordeaux-inp.fr) This paper was presented in part at IEEE ICASSP'15 and EUSIPCO' 15. The work has been partially funded by the Catalan and Spanish governments under grants 2014SGR1567 and TEC2014-59255-C3-1-R. between the different entries of the random vector $\mathbf{y}_{n}$ is then trivially formulated as a binary hypothesis test ${ }^{1}$

$$
\begin{aligned}
& \mathcal{H}_{0}: \mathbf{R}_{M}=\operatorname{dg}\left(\mathbf{R}_{M}\right) \\
& \mathcal{H}_{1}: \mathbf{R}_{M} \neq \operatorname{dg}\left(\mathbf{R}_{M}\right)
\end{aligned}
$$

where $\operatorname{dg}\left(\mathbf{R}_{M}\right)=\mathbf{R}_{M} \odot \mathbf{I}_{M}$ and where $\odot$ denotes Hadamard product and $\mathbf{I}_{M}$ the $M$-dimensional identity matrix.

The problem is equivalent to testing whether the matrix

$$
\mathbf{C}_{M}=\mathbf{D}_{M}^{-1 / 2} \mathbf{R}_{M} \mathbf{D}_{M}^{-1 / 2}
$$

is equal to the identity, where $(\cdot)^{1 / 2}$ denotes the positive square root and $\mathbf{D}_{M}=\operatorname{dg}\left(\mathbf{R}_{M}\right)$. This matrix is typically referred to as the "correlation matrix" of the observations in the statistics literature, although the name "coherence matrix" is more often used in the signal processing field. The diagonal entries of $\mathbf{C}_{M}$ are all equal to one, whereas the off-diagonal values are the correlation coefficients between different spatial entries of the observations.

Typically, the correlation test problem is solved by constructing a statistic based on a sample version of the correlation matrix defined above. More precisely, let $\mathbf{Y}_{M}$ denote an $M \times N$ matrix gathering the $N$ observation samples, i.e. $\mathbf{Y}_{M}=\left[\mathbf{y}_{1}, \ldots, \mathbf{y}_{N}\right]$. We define $\hat{\mathbf{R}}_{M}=\frac{1}{N} \mathbf{Y}_{M} \mathbf{Y}_{M}^{H}$ as the sample covariance matrix and $\hat{\mathbf{D}}_{M}=\hat{\mathbf{R}}_{M} \odot \mathbf{I}_{M}$ its diagonal. The sample correlation matrix (also sample coherence matrix) takes the form

$$
\hat{\mathbf{C}}_{M}=\hat{\mathbf{D}}_{M}^{-1 / 2} \hat{\mathbf{R}}_{M} \hat{\mathbf{D}}_{M}^{-1 / 2} .
$$

Here again, the diagonal entries of $\hat{\mathbf{C}}_{M}$ are all equal to one, whereas the off-diagonal entries are the Pearson's correlation coefficients between different rows of $\mathbf{Y}_{M}$. Correlation tests typically reject the null hypothesis based on a certain statistic that somehow measures how high the magnitudes of the offdiagonal entries of $\hat{\mathbf{C}}_{M}$ are. Commonly proposed statistics in the literature include: the maximum of the off-diagonal entries of $\hat{\mathbf{C}}_{M}$ [3] (usually referred to as the coherence of the random matrix $\mathbf{Y}_{M}$ ), the maximum eigenvalue of $\hat{\mathbf{C}}_{M}$ [4], the squared sum of the off-diagonal entries of $\hat{\mathbf{C}}_{M}$ [5] or the determinant of $\hat{\mathbf{C}}_{M}$ [6], among others.

Traditional statistical analysis of these tests -or, more generally, of the entries of $\hat{\mathbf{C}}_{M^{-}}$have mainly been established the large sample size scenario [7], whereby $N \rightarrow \infty$ for fixed $M$. However, practical applications must typically work with a sample size $N$ than is not much higher (or even lower) than the observation dimension $(M)$. In these situations, classical large sample volume approximations are not accurate anymore, and

\footnotetext{
${ }^{1}$ Note that the diagonal entries of the covariance matrix $\mathbf{R}_{M}$ may be different depending on the hypothesis.
} 
other asymptotic settings that allow both $N$ and $M$ to increase without bound become much more relevant. In this type of asymptotic approach, the two parameters are allowed to grow while being comparable in magnitude, typically by forcing their quotient to converge to a positive constant, namely $M / N \rightarrow c, 0<c<\infty$. Most of the recent works in the statistics literature have analyzed the correlation test problem from the perspective of this alternative asymptotic setting.

In particular, it was shown in [3] that under $\mathcal{H}_{0}$ the empirical distribution of eigenvalues of the sample correlation matrix converges to a Marchenko-Pastur distribution when both $N$ and $M$ grow to infinity but $M / N \rightarrow c, 0<c<\infty$. This result is exactly the same as for the sample covariance matrix, and implies that the normalization by the diagonal $\hat{\mathbf{D}}_{M}$ does not affect the asymptotic eigenvalue distribution. Here, we will see that a similar result holds also under $\mathcal{H}_{1}$. Additionally, [8], [9] established the fact that under $\mathcal{H}_{0}$ the maximum and minimum eigenvalues of $\hat{\mathbf{C}}_{M}$ asymptotically fluctuate according to a Tracy-Widom law, just as it happens in the sample covariance matrix case. This result turns out to be quite useful in order to design the correlation test that rejects the null hypothesis for sufficiently large values of the maximum eigenvalue of $\hat{\mathbf{C}}_{M}$, as proposed in [4]. Another correlation test statistic that has recently been characterized in this high observation asymptotic setting is the coherence of the matrix $\mathbf{Y}_{M}$, defined as the maximum magnitude of the off-diagonal entries of $\hat{\mathbf{C}}_{M}$. It was shown in [10] that when $M, N \rightarrow \infty$ at the same rate, a normalized version of this statistic (multiplied by $\sqrt{N / \log N}$ ) converges almost surely to 2 and asymptotically fluctuates as a Type I extreme distribution. Later, this result has been refined and generalized to less restrictive assumptions [11], [12], [13], [14].

In this paper, we are concerned with somewhat different statistics of the sample correlation matrix, namely those that make use the whole spectrum of the sample correlation matrix. One classical correlation test that falls within this class is the Generalized Likelihood Ratio Test (GLRT) under Gaussian observations. The GLRT statistic is constructed by considering the quotient between the probability densities of the observation $\mathbf{Y}_{M}$ under the two hypotheses (denoted as $f_{1}\left(\mathbf{Y}_{M}\right)$ and $f_{0}\left(\mathbf{Y}_{M}\right)$ ), which in the zero-mean Gaussian case can be written as

$$
\begin{aligned}
& \frac{1}{M N} \log \frac{f_{1}\left(\mathbf{Y}_{M}\right)}{f_{0}\left(\mathbf{Y}_{M}\right)}= \\
= & -\frac{1+\varsigma}{2 M}\left[\log \operatorname{det} \mathbf{C}_{M}+\operatorname{tr}\left[\hat{\mathbf{R}}_{M} \mathbf{R}_{M}^{-1}\right]-\operatorname{tr}\left[\hat{\mathbf{D}}_{M} \mathbf{D}_{M}^{-1}\right]\right]
\end{aligned}
$$

where $\varsigma$ is a boolean variable fixed as $\varsigma=0$ for real-valued observations and $\varsigma=1$ for complex, circularly symmetric observations. Given the fact that the above statistic cannot be constructed (since $\mathbf{R}_{M}$ and $\mathbf{D}_{M}$ are unknown), the GLRT proposes to an ad-hoc alternative obtained by replacing these two matrices by their Maximum Likelihood estimates, which are given by $\hat{\mathbf{R}}_{M}$ and $\hat{\mathbf{D}}_{M}$ respectively [6].

Thus, assuming $N>M$, the GLRT rejects the null hypothesis for large values of the following statistic

$$
\hat{\eta}_{M}^{G L R T}=\frac{-1}{M} \log \operatorname{det}\left(\hat{\mathbf{C}}_{M}\right)
$$

Keeping in mind that the arithmetic mean of the eigenvalues of $\hat{\mathbf{C}}_{M}$ is equal to 1 , one can readily identify the above statistic as a measure of how disperse the eigenvalues of $\hat{\mathbf{C}}_{M}$ are. Clearly, a sample correlation matrix with very distinct eigenvalues will indicate a clear departure from the identity matrix, which will in turn imply that the observations are highly correlated.

There exist multiple extensions of the GLRT to more general statistical observation structures [15], [16], [17]. One of the most studied generalizations of this test is testing whether several components of a Gaussian vector are correlated, namely Wilks' test [18]. Note that the above statistic is a particularization of [18] to the case where the tested components are all scalars. This generalization of the above GLRT has been asymptotically studied under $\mathcal{H}_{0}$ when the dimension of the tested vector signals scales up with the sample size [19], [20], whereas the number of tested components remains constant. This approach is different from the one that will be taken here, in the sense that we will allow the number of tested components $(M)$ to scale up with the sample size.

The GLRT does not really exist in the undersampled scenario $(N<M)$, because $f_{1}\left(\mathbf{Y}_{M}\right)$ is not bounded in the set of positive Hermitian matrices. In order to tackle this problem, it is customary to heuristically adapt the GLRT obtained in the oversampled regime by simply changing the role of $M$ and $N$ in the original test (see, e.g. [21], [22]). The idea is equivalent to considering only the positive eigenvalues of $\hat{\mathbf{C}}_{M}$ in the GLRT, which in our case leads to

$$
\hat{\eta}_{M}^{G L R T}=\frac{-1}{M} \sum_{m=[M-N]^{+}+1}^{M} \log \hat{\lambda}_{m}
$$

where $[x]^{+}=\min (x, 0)$. From now on, we will consider this extended version of the GLRT, which is equally valid in the undersampled $(N<M)$ and oversampled $(N>M)$ situations.

It is well known that the above correlation GLRT can be outperformed by other alternative heuristic tests that, while still exploiting the whole spectrum of $\hat{\mathbf{C}}_{M}$, directly examine the magnitude of the off-diagonal entries of $\hat{\mathbf{C}}_{M}$. As explained above, one such consists in measuring the total squared magnitude of the off-diagonal elements of the sample correlation matrix. Since the diagonal entries of $\hat{\mathbf{C}}_{M}$ are all identically one, this this is equivalent to checking the squared magnitude of the entries of the whole matrix. One popular choice to measure these magnitudes is the Frobenius Norm Test [5], which rejects the null hypothesis for sufficiently large values of

$$
\hat{\eta}_{M}^{F N T}=\frac{1}{M}\left\|\hat{\mathbf{C}}_{M}\right\|_{F}^{2}=\frac{1}{M} \operatorname{tr}\left[\hat{\mathbf{C}}_{M}^{2}\right] .
$$

This test was proposed in [1] as an approximation of the GLRT for low values of the cross-correlation coefficients under $\mathcal{H}_{1}$ and was recently shown to be a locally most powerful invariant test (LMPIT) for the correlation detection problem [23]. The asymptotic behavior of $\hat{\eta}_{M}^{F N}$ under $\mathcal{H}_{0}$ was examined in [5] under the assumption that both $M$ and $N$ tend to infinity while $M / N \rightarrow c, 0<c<\infty$. More specifically, it can be shown from [5] that the statistic in (4) asymptotically fluctuates around the value $1+c$ as a Gaussian random variable. 
Here, we will prove that this is also the case when $\mathcal{H}_{1}$ holds, and we will determine the asymptotic means and covariances under either one of the hypotheses.

By examining the form of (2) and (4), we can come to the conclusion that both tests are based on linear combinations of a function of the eigenvalues of the sample correlation matrix. Indeed, one can write both tests as

$$
\hat{\eta}_{M}=\frac{1}{M} \sum_{m=1}^{M} f\left(\hat{\lambda}_{m}\right)
$$

for some specific choices of the function $f(z)$, namely $f(z)=$ $z^{2}$ in (4) and

$$
f(z)=\left\{\begin{array}{cc}
-\log z & z \neq 0 \\
0 & z=0
\end{array}\right.
$$

in (2). The random quantities that can be formulated as in (5) are generally referred to as Linear Spectral Statistics (LSS) of the sample correlation matrix. The objective of this paper is to provide a complete asymptotic description of this class of statistics, by characterizing their convergence and fluctuations in the general regime where both the sample size $(N)$ and the observation dimension $(M)$ are large but comparable in magnitude. In particular, we will establish that the LSS $\hat{\eta}_{M}$ in (5) is asymptotically close to a deterministic sequence $\bar{\eta}_{M}$, in the sense that $\hat{\eta}_{M}-\bar{\eta}_{M} \rightarrow 0$ almost surely as $M, N \rightarrow \infty$ at the same rate. Furthermore, we will also prove that $\hat{\eta}_{M}$ asymptotically fluctuates around $\bar{\eta}_{M}$ as a Gaussian random variable, and we will provide closed form simple expressions for its asymptotic mean and covariance. These results will be particularized to the two LSS cases presented above, namely the FNT and the GLRT. Results will be valid under both $\mathcal{H}_{0}$ and $\mathcal{H}_{1}$, so they can be used both to establish the threshold level that guarantees a certain asymptotic probability of false alarm, as well as to study the asymptotic power of the tests.

It should be pointed out that our study for LSS of the sample correlation matrix is quite similar to the one carried out in [24] for the sample covariance matrix and later extended to the GLRT for non-Gaussian observations under $\mathcal{H}_{0}$ in [25], [26] and to the non-centered case [27]. In fact, it will be shown in the following sections that (under the centered Gaussian assumption) the LSS based on these two matrices have an equivalent first order behavior, whereas the second order asymptotic fluctuations turn out to be substantially different. Our study reveals that both quantities asymptotically fluctuate as Gaussian random variables, but with different means and variances. This means that the normalization by the diagonal random matrices $\hat{\mathbf{D}}_{M}^{-1 / 2}$ in (1) is equivalent to the normalization by the deterministic $\mathbf{D}_{M}^{-1 / 2}$ only as far as the first order asymptotic behavior is concerned. A substantially different behavior is observed in terms of fluctuations depending on whether random or deterministic normalization is employed.

On the other hand, it was recently brought to our attention that a similar study as the one presented here has recently been carried out in [28]. This paper also analyzes the asymptotic behavior of LSS of sample correlation matrices under the same asymptotic conditions. The results in [28] are derived under general (not necessarily Gaussian) observations, although the asymptotic description is only valid under the null hypothesis $\mathcal{H}_{0}$. Here, by resorting to the Gaussian assumption, we have been able to provide a more general result that encompasses both $\mathcal{H}_{0}$ and $\mathcal{H}_{1}$. Furthermore, our expressions for the asymptotic mean and variance of the LSS can be easily particularized to the FNT and the GLRT presented above, resulting in a very simple description of the asymptotic behavior of these statistics.

Finally, it is worth pointing out the existence of correlation detection tests based on the linear transformations of the sample covariance matrix (see, e.g. [29]) instead of the sample correlation one. In some cases, one may resort to the asymptotic results in [24] in order to analyze the asymptotic performance of these tests and compare them with the class of LSS considered here. We will not carry out such comparison study in this paper, although this is an interesting subject for further study.

The rest of the paper is organized as follows. In Section II we establish the almost sure convergence of the LSS under the general setting where the observations are possibly correlated. Section III then presents the main result of this paper, namely the CLT that establishes the Gaussianity of the LSS. These results are then particularized to the FNT and the GLRT statistics. The proof of this theorem is presented in IV, although most of the technical derivations have been relegated to the appendices.

Notation The $M \times M$ identity matrix is denoted by $\mathbf{I}_{M}$ and $\mathbf{e}_{m}$ is its $m$ th column. The symbols $\otimes$ and $\odot$ denote Kronecker and Hadamard (element-wise) product between matrices. Furthermore, $\operatorname{vec}(\mathbf{A})$ is a column vector formed by piling the columns of $\mathbf{A}$ on top of each other and $\operatorname{dgvec}(\mathbf{A})$ is a diagonal matrix with diagonal entries given by $\operatorname{vec}(\mathbf{A})$. On the other hand, $\operatorname{vdg}(\mathbf{A})$ is a column vector constructed using the diagonal entries of the square matrix A. Given a matrix $\mathbf{A},\|\mathbf{A}\|$ denotes its spectral norm and, assuming $\mathbf{A}$ square of dimensions $M \times M$, we will write $\operatorname{dg}(\mathbf{A})=\mathbf{A} \odot \mathbf{I}_{M}$. For a given random variable $X$, its expectation is denoted as $\mathbb{E}[X]$ and its variance as $\operatorname{var}(X)$, whereas $\mathbb{P}[\mathcal{A}]$ is the probability of a certain event $\mathcal{A}$.

The following lemma will be of constant use throughout this paper.

Lemma 1: For any two matrices $\mathbf{A}$ and $\mathbf{B}$ of the same dimensions, we have

$$
\begin{gathered}
\|\mathbf{A B}\| \leq\|\mathbf{A}\|\|\mathbf{B}\| \\
\|\mathbf{A}+\mathbf{B}\| \leq\|\mathbf{A}\|+\|\mathbf{B}\| \\
\|\mathbf{A} \otimes \mathbf{B}\|=\|\mathbf{A}\|\|\mathbf{B}\| .
\end{gathered}
$$

If $\mathbf{A}, \mathbf{B}$ are generic $M \times M$ matrices and $\mathbf{B}$ is Hermitian positive semidefinite, we have

$$
\left|\frac{1}{M} \operatorname{tr}[\mathbf{A B}]\right| \leq\|\mathbf{A}\| \frac{1}{M} \operatorname{tr}[\mathbf{B}] \leq \max _{i}\{\mathbf{B}\}_{i i}\|\mathbf{A}\| .
$$

and

$$
\|\mathbf{A} \odot \mathbf{B}\| \leq \max _{i}\{\mathbf{B}\}_{i i}\|\mathbf{A}\| .
$$

Proof: The first four statements are quite conventional [30]. For the last one, see [31, Theorem 5.5.18]. 


\section{Almost Sure CONVERGENCE OF THE LSS}

This section will characterize the asymptotic behavior of the LSS described in (5). Let $\gamma_{1}<\ldots<\gamma_{\bar{M}}$ and $K_{1}, \ldots, K_{\bar{M}}$ denote the $\bar{M}$ different eigenvalues of the true correlation matrix $\mathbf{C}_{M}$ and their associated multiplicities, where clearly $1 \leq \bar{M} \leq M$. In the classical regime, whereby $N \rightarrow \infty$ for fixed $M$, one can easily show that

$$
\hat{\eta}_{M}-\eta_{M} \rightarrow 0
$$

almost surely, where

$$
\eta_{M} \equiv \frac{1}{M} \sum_{m=1}^{\bar{M}} K_{m} f\left(\gamma_{m}\right) .
$$

We will see in this that this is not the case when the observation dimension $M$ is allowed to scale up with the sample size $N$. In this situation, we will see that the random variable $\hat{\eta}_{M}$ has an asymptotic deterministic equivalent, $\bar{\eta}_{M}$, such that the difference $\hat{\eta}_{M}-\bar{\eta}_{M}$ converges to zero almost surely as $M, N$ increase without bound at the same rate. In general terms, the deterministic equivalent $\bar{\eta}_{M}$ is generally different from the quantity $\eta_{M}$ above.

We will be making the following assumptions throughout the paper:

(As1) The set of $M$-dimensional observations $\mathbf{y}_{n}, n=$ $1, \ldots, N$ can be expressed as $\mathbf{y}_{n}=\mathbf{R}_{M}^{1 / 2} \mathbf{x}_{n}$ where $\mathbf{x}_{n}, n=$ $1, \ldots, N$, are standardized i.i.d. Gaussian vectors of zero mean and identity covariance matrix. The Boolean variable $\varsigma$ takes on the value $\varsigma=1$ when the observations $\mathbf{y}_{n}$ are real-valued and $\varsigma=0$ when they are complex and circularly symmetric.

(As2) The observation dimension $M$ is a function of $N$. Furthermore, we assume that we are either in the undersampled or the over-sampled regime, so that if $c_{M}=M / N$, we have either

$$
0<\liminf c_{M} \leq \lim \sup c_{M}<1
$$

and the number of observations is always higher then the observation dimension, or

$$
1<\liminf c_{M} \leq \limsup c_{M}<+\infty
$$

so that the number of observations is always lower than the observation dimension.

Note that in the above assumption we are implicitly avoiding the situation where $c_{M}=M / N=1$. This is because the structure of our proof requires that all the positive eigenvalues of the sample correlation matrix stay away from 0 when the dimension of the matrix scales up with the sample size. It is well known that this can only be guaranteed when $c_{M}$ stays bounded away from 1, as guaranteed by (As2). The following assumptions are rather standard in the statistics literature of LSS (see, e.g. [24]):

(As3) The eigenvalues of $\mathbf{R}_{M}$ are contained in a compact interval of the positive real axis, $\mathbb{R}^{+}$, for all $M$.

(As4) The complex function $f(z)$ is analytical on the positive real axis $\mathbb{R}^{+}$.

Assumption (As3) is necessary in order to guarantee that the eigenvalues of $\mathbf{R}_{M}$ (and, by extension those of $\hat{\mathbf{R}}_{M}$ ) stay bounded above and away from zero. These assumptions are necessary to guarantee the weak convergence of the LSS As for (As4), it is not strictly needed (in the sense that all the results in this paper hold for more general functions $f(z)$ ), although the analycity of $f(z)$ greatly simplifies the derivations.

We will first analyze the first order (almost sure) asymptotic behavior of the LSS $\hat{\eta}_{M}$. In this study, it is important to notice that the diagonal entries of the sample covariance matrix converge to the true diagonal entries, even if the observation dimension scales up with the sample size. In other words, we can guarantee that

$$
\left\|\hat{\mathbf{D}}_{M}-\mathbf{D}_{M}\right\| \rightarrow 0
$$

almost surely under $(\mathbf{A} \mathbf{s} \mathbf{1})-(\mathbf{A} \mathbf{s} 3)$, where $\|\cdot\|$ denotes spectral norm and where we recall that $\hat{\mathbf{D}}_{M}$ and $\mathbf{D}_{M}$ are the diagonal matrices constructed from the diagonal entries of $\hat{\mathbf{R}}_{M}$ and $\mathbf{R}_{M}$ respectively (see Appendix A for a proof). A direct consequence of this is the fact that the diagonal entries of $\hat{\mathbf{D}}_{M}$ are almost surely contained on a compact interval of $\mathbb{R}^{+}$ for all $N$ sufficiently large. Indeed, observe that the diagonal entries of $\mathbf{D}_{M}$ are upper and lower bounded by the maximum and minimum eigenvalues of $\mathbf{R}_{M}$ respectively, and that all these eigenvalues are contained in a compact interval of $\mathbb{R}^{+}$ according to (As3). Therefore, the convergence result in (9) readily implies that both $\left\|\hat{\mathbf{D}}_{M}\right\|$ and $\left\|\hat{\mathbf{D}}_{M}^{-1}\right\|$ will be bounded with probability one for all $N$ sufficiently large. This property is used next in order to establish the almost sure location of the positive eigenvalues of $\hat{\mathbf{C}}_{M}$.

Lemma 2: Assume that (As1) - (As3) hold and define the covariance-type random matrix

$$
\widetilde{\mathbf{C}}_{M}=\mathbf{D}_{M}^{-1 / 2} \hat{\mathbf{R}}_{M} \mathbf{D}_{M}^{-1 / 2} .
$$

Then,

$$
\left\|\widetilde{\mathbf{C}}_{M}-\hat{\mathbf{C}}_{M}\right\| \rightarrow 0
$$

almost surely. In particular, there exists $\mathcal{T}$, a compact interval of the positive real axis, such that all the positive eigenvalues of $\hat{\mathbf{C}}_{M}$ are almost surely located inside $\mathcal{T}$ for all $N$ sufficiently large.

Proof: A direct application of Lemma 1 allows us to write

$$
\begin{aligned}
& \left\|\widetilde{\mathbf{C}}_{M}-\hat{\mathbf{C}}_{M}\right\| \leq \\
\leq & \left(\left\|\hat{\mathbf{D}}_{M}^{-1 / 2}\right\|\left\|\widetilde{\mathbf{C}}_{M}\right\|+\left\|\mathbf{D}_{M}^{-1 / 2}\right\|\left\|\hat{\mathbf{C}}_{M}\right\|\right)\left\|\hat{\mathbf{D}}_{M}^{1 / 2}-\mathbf{D}_{M}^{1 / 2}\right\| .
\end{aligned}
$$

By (9) and (As3) we establish that both $\left\|\hat{\mathbf{D}}_{M}^{-1 / 2}\right\|$ and $\left\|\mathbf{D}_{M}^{-1 / 2}\right\|$ are almost surely bounded for sufficiently large $N$. On the other hand, it is proven in [32] that all the positive eigenvalues of the sample covariance matrix $\hat{\mathbf{R}}_{M}$ are almost surely located inside the interval $\mathcal{S}=[a, b]$ for all large $N$, where

$a=\inf _{M}\left(1-\sqrt{c_{M}}\right)^{2}\left\|\mathbf{R}_{M}^{-1}\right\|^{-1}$ and $b=\sup _{M}\left(1+\sqrt{c_{M}}\right)^{2}\left\|\mathbf{R}_{M}\right\|$.

This shows that both $\left\|\widetilde{\mathbf{C}}_{M}\right\|$ and $\left\|\hat{\mathbf{C}}_{M}\right\|$ are bounded with probability one for sufficiently large $M$. On the other hand, 
if $\hat{d}_{k, M}$ and $d_{k, M}$ represent the $k$ th diagonal entries of $\hat{\mathbf{D}}_{M}$ and $\mathbf{D}_{M}$ respectively, we have

$\left|\sqrt{\hat{d}_{k, M}}-\sqrt{d_{k, M}}\right|=\left|\hat{d}_{k, M}-d_{k, M}\right|\left|\sqrt{\hat{d}_{k, M}}+\sqrt{d_{k, M}}\right|^{-1}$

Since both $d_{k, M}$ and $\hat{d}_{k, M}$ are bounded away from zero, we see that $\left\|\hat{\mathbf{D}}_{M}^{1 / 2}-\mathbf{D}_{M}^{1 / 2}\right\| \rightarrow 0$, proving (10). To prove the second part of the lemma, observe again that [32] establishes that all the positive eigenvalues of $\widetilde{\mathbf{C}}_{M}$ are located in an open subset of the interval

$$
\mathcal{T}=\left[\inf _{M}\left(1-\sqrt{c_{M}}\right)^{2} \frac{1}{\theta}-\epsilon, \sup _{M}\left(1+\sqrt{c_{M}}\right)^{2} \theta+\epsilon\right]
$$

where

$$
\theta=\sup \left\|\mathbf{R}_{M}\right\| \sup \left\|\mathbf{R}_{M}^{-1}\right\|
$$

and where $\epsilon$ is small enough so that $\mathcal{T} \subset \mathbb{R}^{+}$. The spectral norm convergence in (10) together with Weyl's inequality establish that the maximum of the absolute differences between the ordered eigenvalues of $\hat{\mathbf{C}}_{M}$ and the ordered eigenvalues of $\widetilde{\mathbf{C}}_{M}$ converges to zero with probability one. Therefore, the positive eigenvalues of $\hat{\mathbf{C}}_{M}$ will also be almost surely located inside $\mathcal{T}$ for all large $N$.

Lemma 2 has an important consequence for the purposes of determining the convergence of the LSS $\hat{\eta}_{M}$. Indeed, let $\widetilde{\eta}_{M}$ be defined as $\hat{\eta}_{M}$, replacing $\hat{\mathbf{C}}_{M}$ with $\widetilde{\mathbf{C}}_{M}$. A direct consequence of Lemma 2 is the fact that, under (As1) - (As4),

$$
\left|\widetilde{\eta}_{M}-\hat{\eta}_{M}\right| \rightarrow 0
$$

almost surely. Indeed, we know from Lemma 2 and [32] that the eigenvalues of both $\hat{\mathbf{C}}_{M}$ and $\widetilde{\mathbf{C}}_{M}$ are almost surely located on the compact interval $\mathcal{T}$ for all $N$ sufficiently large. Furthermore, the convergence in (10) establishes that the maximum of the absolute difference between the ordered eigenvalues of these matrices converges to zero. This, together with the absolute continuity of the LSS function $f(\cdot)$ on the compact $\mathcal{T}$ shows (13).

An important consequence of (13) is the fact that we can establish the first order convergence of LSS of the sample correlation matrix $\hat{\mathbf{C}}_{M}$ by simply studying the LSS of the normalized sample covariance-like matrix $\widetilde{\mathbf{C}}_{M}$. The asymptotic behavior of this type of random matrices is now well understood in the random matrix theory literature, see e.g. [33], [34]. In particular, let $\widetilde{m}_{M}(z)$ denote the Stieltjes transform of the empirical eigenvalue distribution of $\widetilde{\mathbf{C}}_{M}$, defined as

$$
\widetilde{m}_{M}(z)=\frac{1}{M} \operatorname{tr}\left[\left(\widetilde{\mathbf{C}}_{M}-z \mathbf{I}_{M}\right)^{-1}\right]
$$

where $z \in \mathbb{C}^{+}=\{z \in \mathbb{C}: \operatorname{Im} z>0\}$. The following theorem establishes that the random function $\widetilde{m}_{M}(z)$ has an asymptotic deterministic equivalent, in the sense that there exists a deterministic analytic function $\bar{m}_{M}(z)$ such that $\left|\widetilde{m}_{M}(z)-\bar{m}_{M}(z)\right|$ converges to zero with probability one for all $z$ in the upper complex semiplane.

Theorem 1: [33], [34] Let $z \in \mathbb{C}^{+}$and assume that (As1) - (As3) hold. Then, $\left|\widetilde{m}_{M}(z)-\bar{m}_{M}(z)\right| \rightarrow 0$ almost surely, where $\bar{m}_{M}(z)$ is defined as

$$
\bar{m}_{M}(z)=\frac{-1}{z}\left(1-\frac{N}{M}\right)-\frac{N}{M} \frac{1}{\omega_{M}(z)}
$$

and where $\omega_{M}(z)$ is the unique solution to the following equation in $\mathbb{C}^{+}$:

$$
z=\omega_{M}(z)\left(1-\frac{1}{N} \sum_{m=1}^{\bar{M}} K_{m} \frac{\gamma_{m}}{\gamma_{m}-\omega_{M}(z)}\right) .
$$

The deterministic function $\bar{m}_{M}(z)$ can be directly used to establish the first order convergence of the LSS through the integral formula (46). Indeed, by the well-known location properties of $\widetilde{\mathbf{C}}_{M}$ established in [32] we know that for all $N$ sufficiently large, $\widetilde{\eta}_{M}$ can be expressed with probability one as

$$
\widetilde{\eta}_{M}=\frac{1}{2 \pi \mathrm{j}} \oint_{\mathcal{C}^{-}} f(z) \widetilde{m}_{M}(z) d z
$$

where $\mathcal{C}^{-}$is a negatively oriented simple contour independent of $N$ that encloses the set $\mathcal{T}$ and not $\{0\}$. The pointwise convergence in Theorem 1 can easily be established to hold uniformly on $\mathcal{C}$ [24]. This directly establishes the following corollary.

Corollary 1: Under (As1) - (As4), $\left|\hat{\eta}_{M}-\bar{\eta}_{M}\right| \rightarrow 0$ almost surely, where

$$
\bar{\eta}_{M} \equiv \frac{1}{2 \pi \mathrm{j}} \oint_{\mathcal{C}^{-}} f(z) \bar{m}_{M}(z) d z .
$$

It is interesting to particularize this result to the two specific tests introduced in Section I, namely the GLRT and the FNT. In order to obtain a closed form expression for the asymptotic deterministic equivalent of the two corresponding statistics, we introduce here the change of variable proposed in [35]. Indeed, consider the analytical extension of the function $\omega_{M}(z)$ defined in (15) to the set $\mathbb{C} \backslash(\mathcal{T} \cup\{0\})$, the existence of which can easily be established by the Schwarz reflection principle [35]. Define the mapping $z \mapsto \omega=\omega_{M}(z)$ and its inverse $\omega \mapsto z=z_{M}(\omega)$, given by

$$
z_{M}(\omega)=\omega\left(1-\frac{1}{N} \sum_{m=1}^{\bar{M}} K_{m} \frac{\gamma_{m}}{\gamma_{m}-\omega}\right) .
$$

Let $\mathcal{C}_{\omega}^{+}=\omega_{M}\left(\mathcal{C}^{+}\right)$and

$$
F_{M}(\omega)=f\left(z_{M}(\omega)\right) .
$$

We can re-write (17) as

$$
\begin{aligned}
\bar{\eta}_{M}=\left(1-\frac{N}{M}\right) \frac{1}{2 \pi \mathrm{j}} \oint_{\mathcal{C}^{+}} \frac{f(z)}{z} d z \\
+\frac{N}{M} \frac{1}{2 \pi \mathrm{j}} \oint_{\mathcal{C}_{\omega}^{+}} \frac{F_{M}(\omega)}{\omega} \mathcal{D}_{M}(\omega) d \omega
\end{aligned}
$$

where we have defined $\mathcal{D}_{M}(\omega)$ as

$$
\mathcal{D}_{M}(\omega)=\frac{d z_{M}(\omega)}{d \omega}=1-\frac{1}{N} \sum_{m=1}^{\bar{M}} K_{m} \frac{\gamma_{m}^{2}}{\left(\gamma_{m}-\omega\right)^{2}} .
$$

It can be shown [35] that $\mathcal{C}_{\omega}^{+}$is a simple positive contour that encloses all the eigenvalues of the correlation matrix 
$\left\{\gamma_{m}\right\}$. Additionally, in the oversampled regime (7) $\mathcal{C}_{\omega}$ does not enclose $\{0\}$, whereas in the undersampled one $(8)$ it does. Using this information, one can solve the above integrals via classical Cauchy integral calculus.

\section{A. Particularization to the Frobenius Norm Test statistic}

If we set $f(z)=z^{2}$ in the above integral definition of $\bar{\eta}_{M}$, only the second term turns out to be non-zero, and

$$
\bar{\eta}_{M}=\frac{N}{M} \frac{1}{2 \pi \mathrm{j}} \oint_{\mathcal{C}_{\omega}^{+}} \frac{1}{\omega} \mathcal{Q}_{M}(\omega) \mathcal{D}_{M}(\omega) d \omega .
$$

where we have defined

$$
\mathcal{Q}_{M}(\omega)=\omega^{2}\left(1-\frac{1}{N} \sum_{m=1}^{\bar{M}} K_{m} \frac{\gamma_{m}}{\gamma_{m}-\omega}\right)^{2} .
$$

The following lemma leads to the solution of the above integral.

Lemma 3: Let $\mathcal{Q}_{M}(\omega)$ be defined as in (22) and let $\mathcal{C}_{\omega}^{+}$ denote a positively oriented simple contour obtained as $\mathcal{C}_{\omega}^{+}=$ $\omega_{M}\left(\mathcal{C}^{+}\right)$where $\mathcal{C}^{+}$is a simple contour enclosing $\mathcal{T}$ (defined in Lemma 2) and not zero. Then,

$$
\frac{1}{2 \pi \mathrm{j}} \oint_{\mathcal{C}_{\omega}^{+}} \mathcal{Q}_{M}(\omega) d \omega=\frac{2}{N} \sum_{m=1}^{\bar{M}} K_{m} \gamma_{m}^{3}+2 \frac{c_{M}}{N} \sum_{m=1}^{\bar{M}} K_{m} \gamma_{m}^{2} .
$$

Besides, if $\zeta$ is a complex number located in $\mathbb{C} \backslash \mathcal{C}_{\omega}^{+}$, we have

$$
\frac{1}{2 \pi \mathrm{j}} \oint_{\mathcal{C}_{\omega}^{+}} \frac{\mathcal{Q}_{M}(\omega)}{\omega-\zeta} d \omega=\left(\zeta+c_{M}\right)^{2}+\frac{2}{N} \sum_{r=1}^{\bar{M}} K_{r} \gamma_{r}^{2}
$$

if $\zeta$ is enclosed by $\mathcal{C}_{\omega}^{+}$and

$$
\begin{aligned}
\frac{1}{2 \pi \mathrm{j}} \oint_{\mathcal{C}_{\omega}^{+}} \frac{\mathcal{Q}_{M}(\omega)}{\omega-\zeta} d \omega & =2 \frac{1}{N} \sum_{k=1}^{\bar{M}} K_{k} \frac{\gamma_{k}^{2}}{\gamma_{k}-\zeta}\left(\gamma_{k}+c_{M}\right) \\
& -\left(\frac{1}{N} \sum_{m=1}^{\bar{M}} K_{m} \frac{\gamma_{m}^{2}}{\gamma_{m}-\zeta}\right)^{2}
\end{aligned}
$$

when $\zeta$ is not enclosed by $\mathcal{C}_{\omega}^{+}$. Finally, for any $q, 1 \leq q \leq \bar{M}$, we can write

$$
\begin{gathered}
\frac{1}{2 \pi \mathrm{j}} \oint_{\mathcal{C}_{\omega}^{+}} \frac{\mathcal{Q}_{M}(\omega)}{\left(\omega-\gamma_{q}\right)^{2}} d \omega=2\left(\gamma_{q}+c_{M}\right) \\
\frac{1}{2 \pi \mathrm{j}} \oint_{\mathcal{C}_{\omega}^{+}} \frac{\mathcal{Q}_{M}(\omega)}{\left(\omega-\gamma_{q}\right)^{3}} d \omega=1 .
\end{gathered}
$$

Proof: It follows from standard residue calculus together with the fact that the diagonal entries of the correlation matrix are all equal to one.

Using a partial fraction decomposition of $\mathcal{D}_{M}(\omega) / \omega$ in (21) and applying Lemma 3 we readily see that

$$
\bar{\eta}_{M}=\frac{1}{M} \sum_{m=1}^{\bar{M}} K_{m} \gamma_{m}^{2}+c_{M}
$$

Note that this expression is valid for both the undersampled and the oversampled regimes.

\section{B. Particularization to the extended GLRT statistic}

In this case, setting $f(z)$ as in (6), we can readily see that

$$
\bar{\eta}_{M}=\frac{N}{M} \frac{1}{2 \pi \mathrm{j}} \oint_{\mathcal{C}_{\omega}^{-}} \frac{1}{\omega} \mathcal{L}_{M}(\omega) \mathcal{D}_{M}(\omega) d \omega
$$

where we have defined

$$
\mathcal{L}_{M}(\omega)=\log \left[\omega\left(1-\frac{1}{N} \sum_{m=1}^{\bar{M}} K_{m} \frac{\gamma_{m}}{\gamma_{m}-\omega}\right)\right]
$$

and where $\log (\cdot)$ here denotes the principal branch of the complex logarithm. In the above identity we have used the fact that the original contour $\mathcal{C}^{+}$does not enclose $\{0\}$ by definition, and therefore the first term in (19) is identically zero. The above integral is generally difficult to obtain due to the presence of the logarithm. In this paper, we develop an integration technique based on the approach in [36] which is equally valid in the undersampled and oversampled regimes. In order to introduce the results, we consider the $\bar{M}+1$ solutions to the following equation in $\mu$,

$$
\mu\left(1-\frac{1}{N} \sum_{m=1}^{\bar{M}} K_{m} \frac{\gamma_{m}}{\gamma_{m}-\mu}\right)=0
$$

which will be denoted by $\mu_{0}<\mu_{1}<\ldots<\mu_{\bar{M}}$. It can readily be checked that $\mu_{0}=0<\mu_{1}$ in the oversampled regime, whereas $\mu_{0}<0=\mu_{1}$ in the undersampled regime (see [35] for further details). On the other hand, it can be readily seen [35] that the contour $\mathcal{C}_{\omega}$ always encloses the points $\left\{\gamma_{m}, \mu_{m}, m=1, \ldots, \bar{M}\right\}$ but not $\mu_{0}$. In particular, we see that $\mathcal{C}_{\omega}$ encloses zero only in the undersampled regime.

The following proposition essentially provides the result to the integral in (24).

Proposition 1: Let $\mathcal{L}_{M}(\omega)$ be defined as in (25) and assume that $c_{M} \neq 1$. Let $\mathcal{C}_{\omega}^{+}$be a positively oriented simple contour obtained as $\mathcal{C}_{\omega}^{+}=\omega_{M}\left(\mathcal{C}^{+}\right)$, where $\mathcal{C}^{+}$is a simple contour enclosing $\mathcal{T}$ (defined in Lemma 2 ) and not zero. Then,

$$
\frac{1}{2 \pi \mathrm{j}} \oint_{\mathcal{C}_{\omega}^{+}} \mathcal{L}_{M}(\omega) d \omega=\frac{1}{N} \sum_{m=1}^{\bar{M}} K_{m} \frac{\gamma_{m}^{2}}{\gamma_{m}-\mu_{0}} .
$$

On the other hand, if $\zeta$ is a complex number located in $\mathbb{C} \backslash \mathcal{C}_{\omega}^{+}$, we have

$$
\frac{1}{2 \pi \mathrm{j}} \oint_{\mathcal{C}_{\omega}^{+}} \frac{\mathcal{L}_{M}(\omega)}{\omega-\zeta} d \omega=\log \left(\zeta-\mu_{0}\right)
$$

if $\zeta$ is enclosed by $\mathcal{C}_{\omega}$ but $\zeta \notin \cup_{m=1}^{\bar{M}}\left(\mu_{m}, \gamma_{m}\right)$ and

$$
\begin{aligned}
& \frac{1}{2 \pi \mathrm{j}} \oint_{\mathcal{C}_{\omega}^{+}} \frac{\mathcal{L}_{M}(\omega)}{\omega-\zeta} d \omega= \\
& \quad=-\log \left(1-\frac{1}{N} \sum_{m=1}^{\bar{M}} K_{m} \frac{\gamma_{m}^{2}}{\left(\gamma_{m}-\mu_{0}\right)\left(\gamma_{m}-\zeta\right)}\right)
\end{aligned}
$$

if $\zeta$ is not enclosed by $\mathcal{C}_{\omega}$. Finally, for any $q, 1 \leq q \leq \bar{M}$, we may write

$$
\frac{1}{2 \pi \mathrm{j}} \oint_{\mathcal{C}_{\omega}^{+}} \frac{\mathcal{L}_{M}(\omega)}{\left(\omega-\gamma_{q}\right)^{2}} d \omega=\frac{1}{\gamma_{q}-\mu_{0}}
$$


and

$$
\frac{1}{2 \pi \mathrm{j}} \oint_{\mathcal{C}_{\omega}^{+}} \frac{\mathcal{L}_{M}(\omega)}{\left(\omega-\gamma_{q}\right)^{3}} d \omega=-\frac{1}{2\left(\gamma_{q}-\mu_{0}\right)^{2}} .
$$

Proof: See Appendix B.

The integral in (24) can be directly solved by applying the above proposition, differentiating between the undersampled and oversampled regimes. In the oversampled regime, $\mathcal{C}_{\omega}$ does not enclose $\{0\}$ and $\mu_{0}=0$, so that we can write

$$
\bar{\eta}_{M}=-\frac{1}{M} \log \operatorname{det} \mathbf{C}_{M}+\frac{N-M}{M} \log \left|1-\frac{M}{N}\right|+1 .
$$

On the other hand, in the undersampled regime $\mathcal{C}_{\omega}$ always encloses $\mu_{1}=0$ and $\mu_{0}<0$, so that using again Proposition 1 we obtain

$$
\begin{aligned}
\bar{\eta}_{M}=-\frac{1}{M} \log \operatorname{det} \mathbf{C}_{M}+\left(1-\frac{N}{M}\right) \log \left|\mu_{0}\right|+ \\
+\frac{1}{M} \sum_{m=1}^{\bar{M}} K_{m} \log \left|\frac{\gamma_{m}}{\gamma_{m}-\mu_{0}}\right|+\frac{N}{M} .
\end{aligned}
$$

Observe that the GLRT statistic does not become asymptotically close to the quantity $-M^{-1} \log \operatorname{det} \mathbf{C}_{M}$, meaning that the statistic is not a consistent estimator of this quantity in the considered asymptotic regime.

\section{A Central Limit Theorem on the Linear SPECTRAL STATISTICS}

In the past subsection we have seen that, under assumptions (As1) - (As4), the LSS defined in (5) accepts an asymptotic deterministic equivalent, that is a deterministic value $\bar{\eta}_{M}$ for each $M$ such that $\left|\hat{\eta}_{M}-\bar{\eta}_{M}\right| \rightarrow 0$ almost surely. In this section, we will investigate how the statistic $\hat{\eta}_{M}$ fluctuates around $\bar{\eta}_{M}$ in this asymptotic regime. The idea is to derive a result equivalent to the one derived in [24] for LSS of the sample covariance matrix, but characterizing the asymptotic distribution of LSS of the sample correlation matrix. In order to do that, we consider an $L \times 1$ vector of LSS given by

$$
\hat{\boldsymbol{\eta}}_{M}=\left[\hat{\eta}_{M}^{(1)}, \ldots, \hat{\eta}_{M}^{(L)}\right]^{T}
$$

where

$$
\hat{\eta}_{M}^{(\ell)}=\frac{1}{M} \sum_{m=1}^{M} f_{\ell}\left(\hat{\lambda}_{m}\right)
$$

and where $f_{\ell}(z), \ell=1, \ldots, L$, are functions defined according to (As4). In principle, the case $L=1$ would be enough to characterize the asymptotic distribution of a single LSS. However we present here a more general result considering multiple $\operatorname{LSS}(L \geq 1)$ for the sake of completeness and because the derivation is almost identical to the case $L=1$.

Let $\overline{\boldsymbol{\eta}}_{M}$ denote the $L \times 1$ vector of deterministic equivalents, namely

$$
\overline{\boldsymbol{\eta}}_{M}=\left[\bar{\eta}_{M}^{(1)}, \ldots, \bar{\eta}_{M}^{(L)}\right]^{T}
$$

where each $\bar{\eta}_{M}^{(\ell)}$ is defined as in (19) replacing $f(z)$ with $f_{\ell}(z)$. The objective here is to characterize the asymptotic fluctuations of $\hat{\boldsymbol{\eta}}_{M}$ around $\overline{\boldsymbol{\eta}}_{M}$, by establishing a Central Limit Theorem (CLT) on the statistic $M\left(\hat{\boldsymbol{\eta}}_{M}-\overline{\boldsymbol{\eta}}_{M}\right)$. To that effect, we define two quantities that will take the role of the asymptotic mean and covariance matrix respectively.

Let $F_{\ell}(\omega)$ be defined as $f_{\ell}(z)$ after applying the change of variable $z=z_{M}(\omega)$ defined in (18), namely

$$
F_{\ell}(\omega)=f_{\ell}\left(z_{M}(\omega)\right)
$$

and let $\boldsymbol{\Theta}_{M}(\omega)=\left(\mathbf{C}_{M}-\omega \mathbf{I}_{M}\right)^{-1}$. Define $\mathcal{C}^{-}$as a clockwise oriented simple contour that encloses the interval $\mathcal{T}$ defined in Lemma 2 and not zero, and let $\mathcal{C}_{\omega}^{-}$denote $\omega_{M}\left(\mathcal{C}^{-}\right)$, see further Section II. Recall from (As1) that $\varsigma$ is a Boolean variable that takes the value $\varsigma=0$ if the observations are complex-valued and $\varsigma=1$ if they are real-valued. We define $\boldsymbol{\mu}_{M}$ as an $L \times 1$ column vector with $\ell$ th entry equal to

$$
\left\{\boldsymbol{\mu}_{M}\right\}_{\ell}=\frac{1}{2 \pi \mathrm{j}} \oint_{\mathcal{C}_{\omega}^{-}} F_{\ell}(\omega) \bar{\mu}_{M}(\omega) d \omega
$$

where $\bar{\mu}_{M}(\omega)$ is a scalar complex function defined as

$$
\begin{aligned}
& \bar{\mu}_{M}(\omega) \\
& =\varsigma \frac{\frac{1}{N} \operatorname{tr}\left[\mathbf{C}_{M}^{2} \boldsymbol{\Theta}_{M}^{3}(\omega)\right]}{1-\frac{1}{N} \operatorname{tr}\left[\mathbf{C}_{M}^{2} \boldsymbol{\Theta}_{M}^{2}(\omega)\right]} \\
& +\frac{1+\varsigma}{N} \operatorname{tr}\left[\left(\mathbf{C}_{M} \boldsymbol{\Theta}_{M}(\omega)-2 \mathbf{C}_{M}^{2} \boldsymbol{\Theta}_{M}^{2}(\omega)\right) \odot \mathbf{C}_{M} \boldsymbol{\Theta}_{M}(\omega)\right] \\
& +\omega \frac{1+\varsigma}{N} \operatorname{tr}\left[\left(\mathbf{C}_{M} \boldsymbol{\Theta}_{M}^{2}(\omega) \odot \mathbf{C}_{M}\right)\left(\boldsymbol{\Theta}_{M}(\omega) \odot \mathbf{C}_{M}\right)\right]
\end{aligned}
$$

On the other hand, define $\Phi_{M}$ as an $L \times L$ matrix with $(k, \ell)$ th entry equal to

$$
\left\{\Phi_{M}\right\}_{k, \ell}=\frac{-1}{4 \pi^{2}} \oint_{\mathcal{C}_{\omega}^{-}} \oint_{\mathcal{C}_{\omega}^{-}} F_{k}\left(\omega_{1}\right) F_{\ell}\left(\omega_{2}\right) \bar{\sigma}_{M}^{2}\left(\omega_{1}, \omega_{2}\right) d \omega_{1} d \omega_{2}
$$

where $\bar{\sigma}_{M}^{2}\left(\omega_{1}, \omega_{2}\right)$ is a bivariate complex function defined as

$$
\begin{aligned}
\bar{\sigma}_{M}^{2}\left(\omega_{1}, \omega_{2}\right) & =\frac{1+\varsigma}{\left(\omega_{1}-\omega_{2}\right)^{2}}-\frac{1+\varsigma}{N} \operatorname{tr}\left[\mathbf{C}_{M}^{2} \boldsymbol{\Theta}_{M}^{2}\left(\omega_{1}\right) \boldsymbol{\Theta}_{M}^{2}\left(\omega_{2}\right)\right] \\
& +\frac{1+\varsigma}{N} \operatorname{tr}\left[\mathbf{C}_{M} \boldsymbol{\Delta}_{M}\left(\omega_{1}\right) \mathbf{C}_{M} \boldsymbol{\Delta}_{M}\left(\omega_{2}\right)\right]
\end{aligned}
$$

and where have defined

$$
\boldsymbol{\Delta}_{M}(\omega)=\boldsymbol{\Theta}_{M}^{2}(\omega)-\operatorname{dg}\left[\mathbf{C}_{M} \boldsymbol{\Theta}_{M}^{2}(\omega)\right] .
$$

We are now in the position to establish the CLT on the statistic $M\left(\hat{\boldsymbol{\eta}}_{M}-\overline{\boldsymbol{\eta}}_{M}\right)$, which is formulated in what follows.

Theorem 2: Assume that (As1) - (As3) hold, and that the functions $f_{\ell}(z), \ell=1, \ldots, L$, belong to the functional class in (As4). Consider the definitions in (34) and (36), and assume that the column vector $\boldsymbol{\mu}_{M}$ has bounded norm and that all the eigenvalues of $\boldsymbol{\Phi}_{M}$ belong to a compact interval of $\mathbb{R}^{+}$independent of $M$. Then, the random vector $\boldsymbol{\Phi}_{M}^{-1 / 2}\left(M\left(\hat{\boldsymbol{\eta}}_{M}-\overline{\boldsymbol{\eta}}_{M}\right)-\boldsymbol{\mu}_{M}\right)$ converges in law to a multivariate standardized Gaussian distribution, i.e.

$$
\boldsymbol{\Phi}_{M}^{-1 / 2}\left(M\left(\hat{\boldsymbol{\eta}}_{M}-\overline{\boldsymbol{\eta}}_{M}\right)-\boldsymbol{\mu}_{M}\right) \stackrel{\mathcal{L}}{\rightarrow} \mathcal{N}\left(\mathbf{0}, \mathbf{I}_{L}\right) .
$$

Proof: The proof is postponed to Section IV.

As an immediate consequence of this theorem, we see that LSS based on the sample correlation matrix will asymptotically fluctuate as Gaussian random variables, with an asymptotic mean and variance that will generally depend on the selected hypothesis. We next particularize this result to the 
correlation tests presented in Section I to illustrate the practical applicability of Theorem 2 .

It is interesting to compare the expression of the asymptotic mean and variance of the LSS in Theorem 2 with the corresponding expressions established for the CLT of LSS of the sample covariance matrix $\left(\hat{\mathbf{R}}_{M}\right)$ in [24]. More specifically, it was established in [24] that LSS of sample covariance matrices asymptotically fluctuate according to a Gaussian law with mean and variance equal to the first terms on the right hand side of (35) and (37) respectively. The above result shows that the normalization by the diagonals of the sample covariance matrix has no effect on the first order behavior of LSS but completely modifies the asymptotic behavior in terms of fluctuations of the LSS. The last two terms in (35) and (37) introduce the corrections in the asymptotic mean and variance that take into account this normalization effect.

\section{A. Asymptotic Fluctuations of the FNT Statistic}

We recall that the asymptotic equivalent $\bar{\eta}_{M}$ for this statistic has been derived in Section II. Hence, in order to study its asymptotic fluctuations, we only need to particularize Theorem 2 to the specific case where $L=1$ and $f(z)=z^{2}$. We first derive an expression for $\boldsymbol{\mu}_{M}$ and $\boldsymbol{\Phi}_{M}$, which are both scalars in this problem, by solving the corresponding integrals in (34) and (36) respectively. It is shown in Appendix $\mathrm{C}$ that in this situation the asymptotic mean takes the form

$$
\boldsymbol{\mu}_{M}=\frac{1+\varsigma}{N} \operatorname{tr}\left[\left(\mathbf{C}_{M} \odot \mathbf{C}_{M}\right)^{2}\right]-\frac{2+\varsigma}{N} \operatorname{tr}\left[\mathbf{C}_{M}^{2}\right]
$$

whereas the asymptotic variance can be expressed as

$$
\begin{aligned}
\boldsymbol{\Phi}_{M} & =2(1+\varsigma)\left(\frac{1}{N} \operatorname{tr}\left[\mathbf{C}_{M}^{2}\right]\right)^{2}+ \\
& +4 \frac{1+\varsigma}{N} \operatorname{tr}\left[\left(\mathbf{C}_{M}\left(\mathbf{C}_{M}-\operatorname{dg}\left(\mathbf{C}_{M}^{2}\right)\right)\right)^{2}\right]
\end{aligned}
$$

It is also shown in Appendix $C$ that $\sup _{M}\left|\boldsymbol{\mu}_{M}\right|<+\infty$ and $0<\inf _{M}\left|\boldsymbol{\Phi}_{M}\right| \leq \sup _{M}\left|\boldsymbol{\Phi}_{M}\right|<+\infty$. Therefore, we can conclude that the hypotheses in Theorem 2 hold, and therefore the normalized FNT statistic will converge in law to a standard Gaussian random variable. This means that, in practice, we may approximate the law of $\hat{\eta}_{M}$ as a Gaussian random variable with mean $\bar{\eta}_{M}+\boldsymbol{\mu}_{M} / M$ and variance $\boldsymbol{\Phi}_{M} / M^{2}$, with $\bar{\eta}_{M}, \boldsymbol{\mu}_{M}$ and $\boldsymbol{\Phi}_{M}$ given in (23), (38) and (39) respectively.

\section{B. Asymptotic Fluctuations of the GLRT Statistic}

We showed in Section II that the GLRT statistic is almost surely equivalent to a deterministic quantity $\bar{\eta}_{M}$ given by (32)(33) depending on whether we are in the oversampled or the undersampled regime, respectively. We can now characterize the fluctuations of this statistic around this value by particularizing Theorem 2 to the case $L=1$ and $f(z)$ given by (6). It is shown in Appendix $\mathrm{C}$ that the asymptotic mean takes the form

$$
\begin{aligned}
\boldsymbol{\mu}_{M} & =-\frac{\varsigma}{2} \log \left(1-\frac{1}{N} \operatorname{tr}\left[\mathbf{C}_{M}^{2} \boldsymbol{\Theta}_{M}^{2}\left(\mu_{0}\right)\right]\right) \\
& -\frac{1+\varsigma}{2} c_{M}+\frac{\mu_{0}^{2}}{2} \frac{1+\varsigma}{N} \operatorname{tr}\left[\left(\mathbf{C}_{M} \odot \boldsymbol{\Theta}_{M}\left(\mu_{0}\right)\right)^{2}\right] \\
& -\mu_{0} \frac{1+\varsigma}{N} \operatorname{tr}\left[\mathbf{C}_{M} \boldsymbol{\Theta}_{M}\left(\mu_{0}\right) \odot \mathbf{C}_{M} \boldsymbol{\Theta}_{M}\left(\mu_{0}\right)\right]
\end{aligned}
$$

whereas the asymptotic variance can be expressed as

$$
\begin{aligned}
\boldsymbol{\Phi}_{M} & =-(1+\varsigma) \log \left(1-\frac{1}{N} \operatorname{tr}\left[\mathbf{C}_{M}^{2} \boldsymbol{\Theta}_{M}^{2}\left(\mu_{0}\right)\right]\right) \\
& -\frac{1+\varsigma}{N} \operatorname{tr}\left[\mathbf{C}_{M}^{2} \boldsymbol{\Theta}_{M}^{2}\left(\mu_{0}\right)\right] \\
& +\frac{1+\varsigma}{N} \operatorname{tr}\left[\left(\mathbf{C}_{M}\left(\boldsymbol{\Theta}_{M}\left(\mu_{0}\right)-\operatorname{dg}\left[\mathbf{C}_{M} \boldsymbol{\Theta}_{M}\left(\mu_{0}\right)\right]\right)\right)^{2}\right]
\end{aligned}
$$

where we recall that $\boldsymbol{\Theta}_{M}\left(\mu_{0}\right)=\left(\mathbf{C}_{M}-\mu_{0} \mathbf{I}_{M}\right)^{-1}$ and $\mu_{0}$ denotes the smallest solution to (26). It should be pointed out that the above formulas can be greatly simplified in the oversampled regime, whereby $c_{M}<1$ and $\mu_{0}=0$. In this situation, we are able to write

$$
\begin{aligned}
\boldsymbol{\mu}_{M} & =-\frac{\varsigma}{2} \log \left(1-c_{M}\right)-\frac{1+\varsigma}{2} c_{M} \\
\boldsymbol{\Phi}_{M} & =(1+\varsigma)\left[-c_{M}-\log \left(1-c_{M}\right)+\frac{1}{N} \operatorname{tr}\left[\left(\mathbf{C}_{M}-\mathbf{I}_{M}\right)^{2}\right]\right]
\end{aligned}
$$

It is shown in Appendix $C$ that $\sup _{M}\left|\boldsymbol{\mu}_{M}\right|<+\infty$ and $0<\inf _{M}\left|\boldsymbol{\Phi}_{M}\right| \leq \sup _{M}\left|\boldsymbol{\Phi}_{M}\right|<+\infty$ in both undersampled and oversampled regimes. This implies that Theorem 2 is applicable here, and therefore the GLRT statistic asymptotically fluctuates as a Gaussian random variable with the above mean and variance. We may approximate the law of $\hat{\eta}_{M}$ as a Gaussian random variable with mean $\bar{\eta}_{M}+\boldsymbol{\mu}_{M} / M$ and variance $\boldsymbol{\Phi}_{M} / M^{2}$, with $\bar{\eta}_{M}, \boldsymbol{\mu}_{M}$ and $\boldsymbol{\Phi}_{M}$ respectively given by (32), (42) and (43) in the oversampled regime and by (33), (40) and (41) in the undersampled regime.

\section{Numerical Analysis}

To illustrate the accuracy of the above asymptotic approximations, a simple example was considered where the true covariance matrix was equal to the identity matrix under $\mathcal{H}_{0}$. Under $\mathcal{H}_{1}$ the covariance matrix followed a Toeplitz structure, with $(i, j)$ th entry equal to $\left\{\mathbf{R}_{M}\right\}_{i, j}=\rho^{|i-j|}$ for some realvalued $\rho$ fixed to $\rho=0.2$. A total of $10^{5}$ realizations of the GLRT and the FNT statistics where evaluated, and the corresponding false alarm and detection probabilities were evaluated for different values of the threshold $(\alpha)$. Both complex and real-valued observations where considered, and multiple tests were conducted for both undersampled and oversampled scenarios.

Figures 1 to 2 provide a comparison between the simulated probabilities and the asymptotic ones as a function of the threshold $(\alpha)$ in two situations where $M=30, N=40$ and $M=100, N=80$ respectively. The asymptotic probabilities were obtained according to a Gaussian law with mean and variance as established in the past two subsections. Observe 
that the accuracy of the asymptotic approximation clearly increases as $M, N$ become large, and that some slightly better fitting to the Gaussian law is observed in the GLRT for moderate values of $M, N$. Figure 3 represents the probability of detection as a function of the probability of false alarm for the two tests under analysis. As illustrated in this figure, the FNT generally outperforms the GLRT for the whole range of false alarm probabilities.
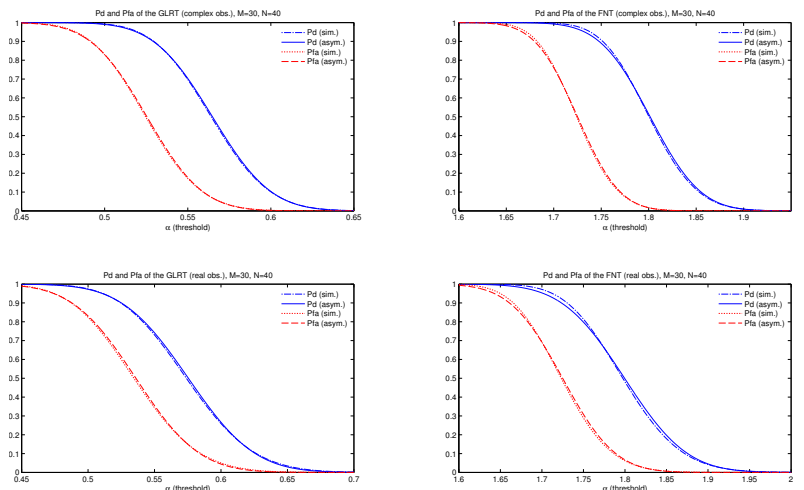

Fig. 1. Simulated versus asymptotic probabilities of false alarm and detection for the GLRT (left hand side) and the FNT (right hand side) when $M=$ $30, N=40$ and $\rho=0.2$. Upper and lower plots respectively correspond to complex and real-valued observations.
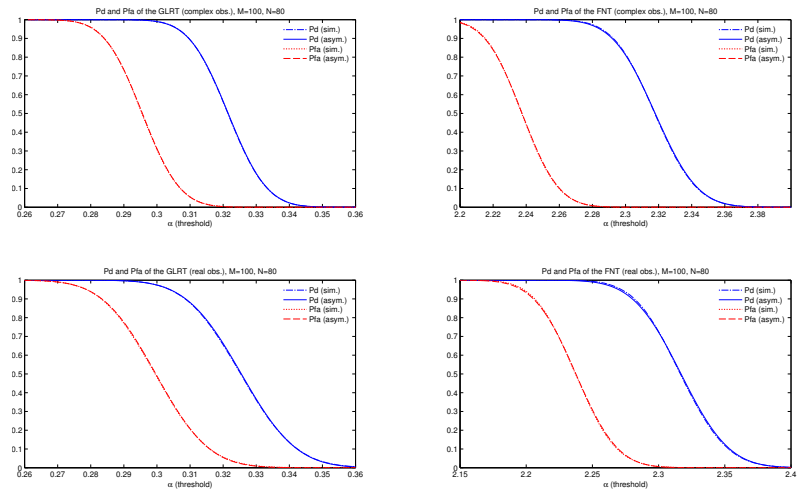

Fig. 2. Simulated versus asymptotic probabilities of false alarm and detection for the GLRT (left hand side) and the FNT (right hand side) when $M=$ $100, N=80$ and $\rho=0.2$. Upper and lower plots respectively correspond to complex and real-valued observations.

\section{Proof of Theorem 2}

We devote this section to the proof of the CLT in Theorem 2. We will essentially follow the methodology established in [37] to derive a CLT on the mutual information of large MIMO channels. The method in question is based on the use of Gaussian tools, which have proven to be extremely useful in order to determine the asymptotic law of functionals of largedimensional random matrices with Gaussian entries. The main idea behind the proof is to establish pointwise convergence of the characteristic function of the statistic $\hat{\eta}_{M}$ towards the characteristic function of a Gaussian random variable.

By the Cramer-Wold device, to show that $M\left(\hat{\boldsymbol{\eta}}_{M}-\overline{\boldsymbol{\eta}}_{M}\right)$ is asymptotically jointly Gaussian distributed, it is sufficient to
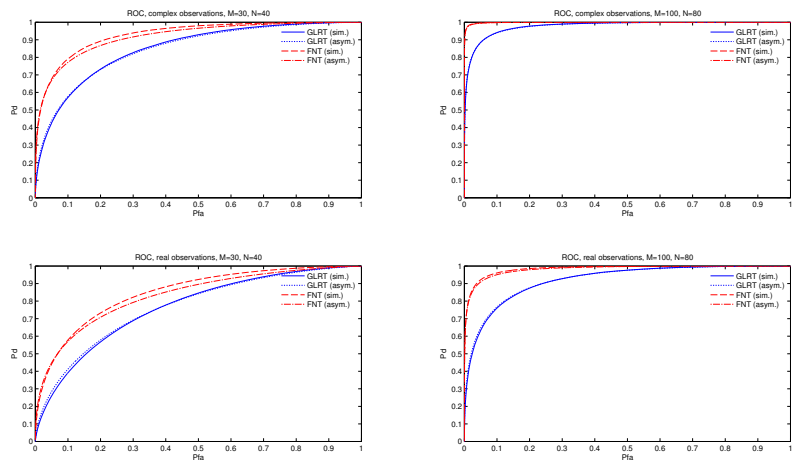

Fig. 3. Probability of detection as a function of the probability of false alarm when $\rho=0.2$ and $M=30, N=40$ (left) or $M=100, N=80$ (right). Upper and lower plots respectively correspond to complex and real-valued observations.

show that $\sum_{\ell=1}^{L} a_{\ell}\left(\hat{\eta}_{M}^{(\ell)}-\bar{\eta}_{M}^{(\ell)}\right)$ is asymptotically Gaussian. for any collection of real numbers $a_{\ell}, \ell=1, \ldots, L$. Consider the function

$$
\Psi_{M}(u)=\exp \left(\mathrm{j} u M \sum_{m=1}^{L} a_{m}\left(\hat{\eta}_{M}^{(m)}-\bar{\eta}_{M}^{(m)}\right)\right) .
$$

Our main objective will be to show that

$$
\mathbb{E}\left[\Psi_{M}(u)\right]-\exp \left(\mathrm{j} \mathbf{a}^{T} \boldsymbol{\mu}_{M} u-\frac{\mathbf{a}^{T} \boldsymbol{\Phi}_{M} \mathbf{a}}{2} u^{2}\right) \rightarrow 0
$$

pointwise in $u$, where $\mathbf{a}=\left[a_{1}, \ldots, a_{L}\right]^{T}$ and where $\boldsymbol{\mu}_{M}$ and $\boldsymbol{\Phi}_{M}$ are as defined in (34) and (36) respectively. By assumption, the norms of $\boldsymbol{\mu}_{M}$ and $\boldsymbol{\Phi}_{M}$ are bounded by a positive quantity independent of $M$. This assumption together with a trivial modification of [37, Proposition 6] will complete the proof. The rest of the section is therefore devoted to showing (45).

The crucial point in the proofs of this paper comes from the fact that, according to (As4), we can rewrite the LSS in (5) in integral form as

$$
\hat{\eta}_{M}^{(m)}=\frac{1}{2 \pi \mathrm{j}} \oint_{\mathcal{C}_{M}^{-}} f_{m}(z) \hat{m}_{M}(z) d z
$$

where we have defined

$$
\hat{m}_{M}(z)=\frac{1}{M} \sum_{m=1}^{M} \frac{1}{\hat{\lambda}_{m}-z}=\frac{1}{M} \operatorname{tr}\left[\hat{\mathbf{D}}_{M} \hat{\mathbf{Q}}_{M}(z)\right]
$$

and

$$
\hat{\mathbf{Q}}_{M}(z)=\left(\hat{\mathbf{R}}_{M}-z \hat{\mathbf{D}}_{M}\right)^{-1}
$$

and where $\mathcal{C}_{M}^{-}$is a negatively (clockwise) oriented simple contour enclosing the positive eigenvalues of $\hat{\mathbf{C}}_{M}$ and not zero. The matrix $\hat{\mathbf{Q}}_{M}(z)$ will play an important role throughout the derivations. In particular, using the spectral norm inequality in Lemma 1 one can establish that

$$
\left\|\hat{\mathbf{Q}}_{M}(z)\right\| \leq\left\|\hat{\mathbf{D}}_{M}^{-1}\right\|\left\|\left(\hat{\mathbf{C}}_{M}-z \mathbf{I}_{M}\right)^{-1}\right\|<\frac{K}{\operatorname{dist}(z, \mathcal{T})}
$$

for some positive constant $K$ independent of $N$.

The first step of the proof consists in replacing the original contour $\mathcal{C}_{M}^{-}$in (46) by a contour $\mathcal{C}^{-}$that does not depend 
on $M$, as in (16). Unfortunately, the large- $M$ representation of $\hat{\eta}_{M}^{(m)}$ obtained by direct replacement of $\mathcal{C}_{M}$ by $\mathcal{C}$ as in (16) is not useful here, because the characteristic function of the resulting random variable may not exist for all $M$. This is because there might exist realizations for which the eigenvalues of $\hat{\mathbf{C}}_{M}$ become dangerously close the contour $\mathcal{C}$ or even on $\mathcal{C}$. In order to overcome this difficulty, we will follow the approach in [38], [39] and consider an equivalent (large- $M$ ) representation of $\hat{\eta}_{M}^{(m)}$ that is guaranteed to have characteristic function for all $M$.

Recall the definition of the support $\mathcal{S}$ in Lemma 2 and define $\mathcal{S}_{\epsilon}=\{x \in \mathbb{R}: \operatorname{dist}(x, \mathcal{S}) \leq \epsilon\}$ for $\epsilon>0$. Assume that $\epsilon$ is small enough such that $\mathcal{S}_{2 \epsilon}$ does not contain $\{0\}$. Let $\phi$ denote a smooth function $\phi: \mathbb{R} \rightarrow[0,1]$ such that $\phi(x)=1$ for $x \in \mathcal{S}_{\epsilon}$ and $\phi(x)=0$ for $x \in \mathbb{R} \backslash \mathcal{S}_{2 \epsilon}$. We will write $\phi_{M}=$ $\operatorname{det} \phi\left(\hat{\mathbf{R}}_{M}\right)$. By [32], we know that $\phi_{M}=1$ with probability one for all $M$ sufficiently large. Therefore, we may represent $\hat{\eta}_{M}^{(m)}$ as

$$
\hat{\eta}_{M}^{(m)}=\frac{1}{2 \pi \mathrm{j}} \oint_{\mathcal{C}^{-}} f^{(m)}(z) \hat{m}_{M}(z) \phi_{M} d z
$$

almost surely for all $M$ sufficiently large. Having introduced this regularization parameter, we are now in the position of introducing the main technical tools that will be used in the proof of Theorem 2. Following the approach in [37], our derivations will be based on the partial integration formula for Gaussian functionals, together with the Poincaré-Nash inequality. We introduce these tools in the following proposition.

Remark 1: In what follows, the symbol $\mathcal{O}\left(N^{-k}\right)$ will denote a general bivariate complex function that is bounded in magnitude by $\epsilon\left(z_{1}, z_{2}\right) N^{-k}$, where $\epsilon\left(z_{1}, z_{2}\right)$ does not depend on $N$ and is such that

$$
\sup _{\left(z_{1}, z_{2}\right) \in \mathcal{C} \times \mathcal{C}}\left\|\epsilon\left(z_{1}, z_{2}\right)\right\|<+\infty .
$$

The function itself may be different from one line to another, and it may be matrix valued, in which case (51) is understood as the spectral norm. On the other hand, $\mathcal{O}\left(N^{-\mathbb{N}}\right)$ should be understood as a bivariate complex function that can be written as $\mathcal{O}\left(N^{-\ell}\right)$ for every $\ell \in \mathbb{N}$.

Proposition 2: Assume that, for each fixed $z \in \mathbb{C}$, the function $\Omega\left(\mathbf{X}, \mathbf{X}^{*}, z\right): \mathbb{R}^{2 M N} \rightarrow \mathbb{C}$ is continuously differentiable and such that both itself and its partial derivatives are polynomically bounded. If $\mathbf{X}$ is real valued, simply consider $\Gamma$ as a function on $\mathbb{R}^{M N}$, with the same properties. Than, under (As1) we can write

$$
\mathbb{E}\left[X_{i j} \Omega\left(\mathbf{X}, \mathbf{X}^{*}, z\right)\right]=\mathbb{E}\left[\frac{\partial \Omega\left(\mathbf{X}, \mathbf{X}^{*}, z\right)}{\partial X_{i j}^{*}}\right]
$$

where $^{2}$

$$
\frac{\partial}{\partial X_{i j}^{*}}=\frac{1+\varsigma}{2} \frac{\partial}{\partial \operatorname{Re}\left[X_{i j}\right]}+\mathrm{j} \frac{1-\varsigma}{2} \frac{\partial}{\partial \operatorname{Im}\left[X_{i j}\right]} .
$$

${ }^{2}$ The second term is understood to be zero when $X_{i j}$ is real-valued $(\varsigma=1)$.
On the other hand, we can also write

$$
\begin{aligned}
\operatorname{var}\left[\Omega\left(\mathbf{X}, \mathbf{X}^{*}, z\right)\right] \leq & \sum_{i=1}^{M} \sum_{j=1}^{N} \mathbb{E}\left|\frac{\partial \Omega\left(\mathbf{X}, \mathbf{X}^{*}, z\right)}{\partial X_{i j}}\right|^{2} \\
& +(1-\varsigma) \mathbb{E}\left|\frac{\partial \Omega\left(\mathbf{X}, \mathbf{X}^{*}, z\right)}{\partial X_{i j}^{*}}\right|^{2}
\end{aligned}
$$

where now

$$
\frac{\partial}{\partial X_{i j}}=\frac{1+\varsigma}{2} \frac{\partial}{\partial \operatorname{Re}\left[X_{i j}\right]}-\mathrm{j} \frac{1-\varsigma}{2} \frac{\partial}{\partial \operatorname{Im}\left[X_{i j}\right]} .
$$

The function $\phi_{M}$ is continuously differentiable (on $\mathbb{R}^{2 M N}$ for complex-valued observations, $\mathbb{R}^{M N}$ for real-valued ones) with polynomically bounded partial derivatives. If, in addition, $\sup _{z \in \mathcal{C}} \mathbb{E}\left(\left|\Omega\left(\mathbf{X}, \mathbf{X}^{*}, z\right) \phi_{M}\right|^{2}\right)<C$ for some positive deterministic $C$ independent of $M$, then under (As1),

$$
\mathbb{E}\left[\Omega\left(\mathbf{X}, \mathbf{X}^{*}, z\right) \phi_{M}^{r}\right]=\mathbb{E}\left[\Omega\left(\mathbf{X}, \mathbf{X}^{*}, z\right) \phi_{M}\right]+\mathcal{O}\left(N^{-\mathbb{N}}\right)
$$

for any $r \in \mathbb{N}$, and also

$$
\mathbb{E}\left[\Omega\left(\mathbf{X}, \mathbf{X}^{*}, z\right) \frac{\partial \phi_{M}}{\partial X_{i j}}\right]=\mathcal{O}\left(N^{-\mathbb{N}}\right)
$$

where the term $\mathcal{O}\left(N^{-\mathbb{N}}\right)$ should be understood as in Remark 1 above.

Proof: The first identity is the integration by parts formula for Gaussian functionals, see [38], [37]. The second one corresponds to the Poincaré-Nash inequality, see [38], [40]. For the rest of the proof, see Appendix E.

The identity in (54) basically states that we can disregard the presence of powers of this regularization factor. One of the conclusions of Proposition 2 is the fact that we can basically ignore the presence of the regularization term $\phi_{M}$ up to an error of order $\mathcal{O}\left(N^{-\ell}\right)$ for any $\ell \in \mathbb{N}$, which will be irrelevant for the purposes of our derivations.

Consider therefore the expectation of the function $\Psi_{M}(u)$ in (44) where $\hat{\eta}_{M}^{(m)}$ takes the form in (50). It can readily be checked that this function is continuously differentiable, and one can express its derivative as

$$
\begin{array}{r}
\frac{d \mathbb{E}\left[\Psi_{M}(u)\right]}{d u}=\mathrm{j} M \sum_{\ell=1}^{L} a_{\ell} \mathbb{E}\left[\left(\hat{\eta}_{M}^{(\ell)}-\bar{\eta}_{M}^{(\ell)}\right) \Psi_{M}(u)\right] \\
=\mathrm{j} \sum_{\ell=1}^{L} a_{\ell} \frac{1}{2 \pi \mathrm{j}} \oint_{\mathcal{C}^{-}} f_{\ell}\left(z_{1}\right) \mathbb{E}\left[\alpha_{M}\left(z_{1}\right) \Psi_{M}(u)\right] d z_{1}
\end{array}
$$

where we have defined

$$
\alpha_{M}(z)=M\left(\hat{m}_{M}(z) \phi_{M}-\bar{m}_{M}(z)\right) .
$$

The main objective of the following derivations is to investigate the asymptotic behavior of the term $M \mathbb{E}\left[\alpha_{M}(z) \Psi_{M}(u)\right]$ in (56) by means of Proposition 2.

First of all, we consider a matrix formulation of the quantity $\alpha_{M}(z)$. In (47), we have introduced an expression of $\hat{m}_{M}(z)$ as a function of the random matrix $\hat{\mathbf{Q}}_{M}(z)$, defined in (48). Using the definition of $\omega_{M}(z)$ in Theorem 1 , we can equivalently express the deterministic equivalent $\bar{m}_{M}(z)$ in (14) as

$$
\bar{m}_{M}(z)=\frac{1}{M} \operatorname{tr}\left[\mathbf{D}_{M} \overline{\mathbf{Q}}_{M}(z)\right]
$$


where we have defined

$$
\overline{\mathbf{Q}}_{M}(z)=\frac{\omega_{M}(z)}{z}\left(\mathbf{R}_{M}-z \mathbf{D}_{M}\right)^{-1} .
$$

Therefore, we can express $\alpha_{M}(z)$ as

$$
\alpha_{M}(z)=\operatorname{tr}\left[\hat{\mathbf{D}}_{M} \hat{\mathbf{Q}}_{M}(z) \phi_{M}-\mathbf{D}_{M} \overline{\mathbf{Q}}_{M}(z)\right] .
$$

The following related quantity will also be useful in the derivations of this section:

$$
\beta_{M}(z)=\operatorname{tr}\left[\mathbf{R}_{M}\left(\hat{\mathbf{Q}}_{M}(z) \phi_{M}-\overline{\mathbf{Q}}_{M}(z)\right)\right] .
$$

In order to handle quantities like $\alpha_{M}(z)$ and $\beta_{M}(z)$, we will repeatedly use the Gaussian tools based on the integration by parts formula in (52) and the Poincaré-Nash inequality in (53). Before going into the technical details, we present first an informal sketch of the rest of the proof.

\section{A. Sketch of the proof}

Let us first provide an informal explanation on how to analyze $\mathbb{E}\left[\alpha_{M}\left(z_{1}\right) \Psi_{M}(u)\right]$ in (56). We consider the two products $\hat{\mathbf{Q}}_{M}(z) \hat{\mathbf{R}}_{M}$ and $\hat{\mathbf{Q}}_{M}(z) \hat{\mathbf{D}}_{M}$ and use the definition of the sample covariance matrix to write

$$
\hat{\mathbf{R}}_{M}=\sum_{i=1}^{M} \sum_{j=1}^{N} X_{i j} \mathbf{R}_{M}^{1 / 2} \frac{\mathbf{e}_{i} \mathbf{x}_{j}^{H}}{N} \mathbf{R}_{M}^{1 / 2}
$$

where $\mathbf{e}_{i}$ is the $i$ th column of the identity $\mathbf{I}_{M}$ and $\mathbf{x}_{j}$ the $j$ th column of X. By inserting the decomposition in (59) into the following expressions (and the equivalent for $\hat{\mathbf{D}}_{M}$ ) and then applying the integration by parts formula in (52), we will be able to write

$$
\begin{array}{r}
\mathbb{E}\left[\hat{\mathbf{Q}}_{M}\left(z_{1}\right) \hat{\mathbf{D}}_{M} \phi_{M} \Psi_{M}(u)\right]=\mathbb{E}\left[\hat{\mathbf{Q}}_{M}\left(z_{1}\right) \mathbf{D}_{M} \phi_{M} \Psi_{M}(u)\right] \\
+(\text { other terms })+\mathcal{O}\left(N^{-\mathbb{N}}\right)
\end{array}
$$

and

$$
\begin{aligned}
& \mathbb{E}\left[\hat{\mathbf{Q}}_{M}\left(z_{1}\right) \hat{\mathbf{R}}_{M} \phi_{M} \Psi_{M}(u)\right]=\frac{z_{1}}{\omega_{M}\left(z_{1}\right)} \times \\
\times & \mathbb{E}\left[\hat{\mathbf{Q}}_{M}\left(z_{1}\right) \mathbf{R}_{M} \phi_{M} \Psi_{M}(u)\right]+(\text { other terms })+\mathcal{O}\left(N^{-\mathbb{N}}\right)
\end{aligned}
$$

Then, combining the above two equations by the trivial identity

$$
\hat{\mathbf{Q}}_{M}\left(z_{1}\right)\left(\hat{\mathbf{R}}_{M}-z_{1} \hat{\mathbf{D}}_{M}\right)=\mathbf{I}_{M}
$$

and right multiplying both sides of the result by $\overline{\mathbf{Q}}_{M}\left(z_{1}\right) \mathbf{D}_{M}$, we will obtain

$$
\begin{aligned}
\mathbb{E}\left[\hat{\mathbf{Q}}_{M}\left(z_{1}\right) \mathbf{D}_{M} \phi_{M} \Psi_{M}(u)\right] & =\overline{\mathbf{Q}}_{M}\left(z_{1}\right) \mathbf{D}_{M} \mathbb{E}\left[\phi_{M} \Psi_{M}(u)\right] \\
& +(\text { other terms })+\mathcal{O}\left(N^{-\mathbb{N}}\right)
\end{aligned}
$$

This can be directly inserted into (60), leading to (after taking traces)

$$
\mathbb{E}\left[\alpha_{M}\left(z_{1}\right) \Psi_{M}(u)\right]=(\text { other terms })+\mathcal{O}\left(N^{-\mathbb{N}}\right) .
$$

By investigating the asymptotic behavior of the "other terms" we will obtain

$$
\begin{gathered}
\mathbb{E}\left[\alpha_{M}\left(z_{1}\right) \Psi_{M}(u)\right]=\frac{\bar{\mu}_{M}\left(\omega_{M}\left(z_{1}\right)\right)}{\mathcal{D}_{M}\left(\omega_{M}\left(z_{1}\right)\right)} \mathbb{E}\left[\Psi_{M}(u)\right]+ \\
+\mathrm{j} u \sum_{m=1}^{L} \frac{a_{m}}{2 \pi \mathrm{j}} \oint_{\mathcal{C}^{-}} \frac{f_{m}\left(z_{2}\right) \bar{\sigma}_{M}^{2}\left(\omega_{M}\left(z_{1}\right), \omega_{M}\left(z_{2}\right)\right)}{\mathcal{D}_{M}\left(\omega_{M}\left(z_{1}\right)\right) \mathcal{D}_{M}\left(\omega_{M}\left(z_{2}\right)\right)} d z_{2} \times \\
\times \mathbb{E}\left[\Psi_{M}(u)\right]+\mathcal{O}\left(N^{-\mathbb{N}}\right)
\end{gathered}
$$

where $\mathcal{D}_{M}(\omega)$ is defined in (20). Inserting this back into (56) and using the change of variables $z \mapsto \omega_{M}(z)$ presented in Section II (see (19)), we will obtain

$\frac{d \mathbb{E}\left[\Psi_{M}(u)\right]}{d u}=\left(j \mathbf{a}^{T} \mu_{M}-u \mathbf{a}^{T} \boldsymbol{\Phi}_{M} \mathbf{a}\right) \mathbb{E}\left[\Psi_{M}(u)\right]+\mathcal{O}\left(N^{-1}\right)$

where the term $\mathcal{O}\left(N^{-1}\right)$ is bounded in $u$ when this variable is confined to a finite interval. Solving the above differential equation, we finally obtain (45) and the proof is complete.

Next, we provide a formal proof to the above statements. In order to facilitate the exposition, we will simplify the notation as indicated in the following remark.

Remark 2: In the rest of this section, we will omit the dependence on $M$ of all quantities. Furthermore, we will write $\omega_{i}=\omega_{M}\left(z_{i}\right), \alpha_{i}=\alpha_{M}\left(z_{i}\right), \beta_{i}=\beta_{M}\left(z_{i}\right), \hat{\mathbf{Q}}_{i}=\hat{\mathbf{Q}}_{M}\left(z_{i}\right)$, $\overline{\mathbf{Q}}_{i}=\overline{\mathbf{Q}}_{M}\left(z_{i}\right), i=1,2$. We will also omit the dependence on $u$ in $\Psi_{M}(u)$. Furthermore, from now on we assume $a_{\ell}=1$ (note that this is done without loss of generality by simply redefining the functions $f_{\ell}(z)$ ).

\section{B. Asymptotic characterization of $\mathbb{E}\left[\alpha_{M}\left(z_{1}\right) \Psi_{M}(u)\right]$}

We will follow the approach that has been outlined in the above sketch of the proof. We begin by analyzing (61), inserting the decomposition in (59) and applying (52), (54) and (55), namely

$$
\begin{aligned}
& \mathbb{E}\left[\hat{\mathbf{Q}}_{1} \hat{\mathbf{R}} \Psi \phi\right] \\
& =\sum_{i=1}^{M} \sum_{j=1}^{N} \mathbb{E}\left[X_{i j} \hat{\mathbf{Q}}_{1} \mathbf{R}^{1 / 2} \frac{\mathbf{e}_{i} \mathbf{x}_{j}^{H}}{N} \mathbf{R}^{1 / 2} \Psi \phi\right] \\
& =\mathbb{E}\left[\hat{\mathbf{Q}}_{1} \mathbf{R} \Psi \phi\right]-\mathbb{E}\left[\hat{\mathbf{Q}}_{1} \hat{\mathbf{R}} \Psi \phi \frac{1}{N} \operatorname{tr}\left[\mathbf{R} \hat{\mathbf{Q}}_{1} \phi\right]\right] \\
& -\varsigma \frac{1}{N} \mathbb{E}\left[\hat{\mathbf{Q}}_{1} \mathbf{R} \hat{\mathbf{Q}}_{1} \hat{\mathbf{R}} \Psi \phi\right]+\frac{z_{1}(1+\varsigma)}{N} \mathbb{E}\left[\hat{\mathbf{Q}}_{1} \operatorname{dg}\left(\hat{\mathbf{Q}}_{1} \mathbf{R}\right) \hat{\mathbf{R}} \Psi \phi\right] \\
& -\mathrm{j}(1+\varsigma) u c_{M} \sum_{m=1}^{L} \frac{1}{2 \pi \mathrm{j}} \oint_{\mathcal{C}^{-}} f_{m}\left(z_{2}\right) \mathbb{E}\left[\hat{\mathbf{Q}}_{1} \mathbf{R} \hat{\mathbf{\Upsilon}}_{2} \hat{\mathbf{R}} \Psi \phi\right] d z_{2} \\
& +\mathcal{O}\left(N^{-\mathbb{N}}\right)
\end{aligned}
$$

where we have defined

$$
\hat{\mathbf{\Upsilon}}_{2}=\hat{\mathbf{Q}}_{2} \hat{\mathbf{D}} \hat{\mathbf{Q}}_{2}-\operatorname{dg}\left(\hat{\mathbf{Q}}_{2} \hat{\mathbf{R}} \hat{\mathbf{Q}}_{2}\right)
$$


In order to obtain this expression, we used the fact that the partial derivatives of $\hat{\mathbf{R}}$ and $\hat{\mathbf{Q}}_{1}$ with respect to $X_{i j}^{*}$ are

$$
\begin{aligned}
\frac{\partial \hat{\mathbf{R}}}{\partial X_{i j}^{*}} & =\frac{1}{N} \mathbf{R}^{1 / 2}\left[\mathbf{x}_{j} \mathbf{e}_{i}^{T}+\varsigma \mathbf{e}_{i} \mathbf{x}_{j}^{H}\right] \mathbf{R}^{1 / 2} \\
\frac{\partial \hat{\mathbf{Q}}_{1}}{\partial X_{i j}^{*}} & =-\frac{1}{N} \hat{\mathbf{Q}}_{1} \mathbf{R}^{1 / 2}\left[\mathbf{x}_{j} \mathbf{e}_{i}^{T}+\varsigma \mathbf{e}_{i} \mathbf{x}_{j}^{H}\right] \mathbf{R}^{1 / 2} \hat{\mathbf{Q}}_{1} \\
& +\frac{z_{1}(1+\varsigma)}{N} \hat{\mathbf{Q}}_{1} \operatorname{dg}\left(\mathbf{R}^{1 / 2} \mathbf{x}_{j} \mathbf{e}_{i}^{T} \mathbf{R}^{1 / 2}\right) \hat{\mathbf{Q}}_{1} .
\end{aligned}
$$

Next, we observe that the second term on the right hand side of (65) can be expressed as

$$
\begin{aligned}
\mathbb{E}\left[\hat{\mathbf{Q}}_{1} \hat{\mathbf{R}} \Psi \phi \frac{1}{N} \operatorname{tr}\left[\mathbf{R} \hat{\mathbf{Q}}_{1}\right]\right] & =\mathbb{E}\left[\hat{\mathbf{Q}}_{1} \hat{\mathbf{R}} \Psi \phi\right] \frac{1}{N} \operatorname{tr}\left[\mathbf{R} \overline{\mathbf{Q}}_{1}\right] \\
& +\frac{1}{N} \mathbb{E}\left[\hat{\mathbf{Q}}_{1} \hat{\mathbf{R}} \Psi \phi \beta_{1}\right]
\end{aligned}
$$

where $\beta_{1}$ is defined in (58). Note that the term $\mathbb{E}\left[\hat{\mathbf{Q}}_{1} \hat{\mathbf{R}} \Psi \phi\right]$ that appears on the right hand side of the above equation coincides with the one on the left hand side of (65). We can insert the above identity back into (65) and use the fact that

$$
1+\frac{1}{N} \operatorname{tr}\left[\mathbf{R} \overline{\mathbf{Q}}_{1}\right]=\frac{\omega_{1}}{z_{1}}
$$

which follows from the definition of $\omega_{1}$ in (15). Since the integration contour does not contain $z=0$, we can ensure that (see Appendix F)

$$
\inf _{M} \inf _{z_{1} \in \mathcal{C}}\left|\omega_{M}\left(z_{1}\right)\right|>0 .
$$

Therefore we can freely multiply both sides of the resulting equation by $\frac{z_{1}}{\omega_{1}}$ and write

$$
\begin{aligned}
& \mathbb{E}\left[\hat{\mathbf{Q}}_{1} \hat{\mathbf{R}} \Psi \phi\right] \\
& =\frac{z_{1}}{\omega_{1}} \mathbb{E}\left[\hat{\mathbf{Q}}_{1} \mathbf{R} \Psi \phi\right]-\frac{z_{1}}{\omega_{1}} \frac{1}{N} \mathbb{E}\left[\hat{\mathbf{Q}}_{1} \hat{\mathbf{R}} \Psi \phi \beta_{1}\right] \\
& -\varsigma \frac{z_{1}}{\omega_{1}} \frac{1}{N} \mathbb{E}\left[\hat{\mathbf{Q}}_{1} \mathbf{R} \hat{\mathbf{Q}}_{1} \hat{\mathbf{R}} \Psi \phi\right] \\
& +\frac{z_{1}^{2}}{\omega_{1}} \frac{(1+\varsigma)}{N} \mathbb{E}\left[\hat{\mathbf{Q}}_{1} \operatorname{dg}\left(\hat{\mathbf{Q}}_{1} \mathbf{R}\right) \hat{\mathbf{R}} \Psi \phi\right] \\
& -\mathrm{j} \frac{z_{1}}{\omega_{1}} u \sum_{m=1}^{L} \frac{1}{2 \pi \mathrm{j}} \oint_{\mathcal{C}^{-}} f_{m}\left(z_{2}\right) \frac{1+\varsigma}{N} \mathbb{E}\left[\hat{\mathbf{Q}}_{1} \mathbf{R} \hat{\mathbf{\Upsilon}}_{2} \hat{\mathbf{R}} \Psi \phi\right] d z_{2} \\
& +\mathcal{O}\left(N^{-\mathbb{N}}\right) .
\end{aligned}
$$

On the other hand, a similar derivation can be performed with $\mathbb{E}\left[\hat{\mathbf{Q}}_{1} \hat{\mathbf{D}} \Psi \phi\right]$, which gives

$$
\begin{aligned}
& \mathbb{E}\left[\hat{\mathbf{Q}}_{1} \hat{\mathbf{D}} \Psi \phi\right] \\
& =\mathbb{E}\left[\hat{\mathbf{Q}}_{1} \mathbf{D} \Psi \phi\right]-\frac{1}{N} \mathbb{E}\left[\hat{\mathbf{Q}}_{1} \hat{\mathbf{R}} \operatorname{dg}\left(\mathbf{R} \hat{\mathbf{Q}}_{1}\right) \Psi \phi\right] \\
& -\varsigma \frac{1}{N} \mathbb{E}\left[\hat{\mathbf{Q}}_{1} \mathbf{R} \operatorname{dg}\left(\hat{\mathbf{R}} \hat{\mathbf{Q}}_{1}\right) \Psi \phi\right] \\
& +(1+\varsigma) z_{1} \frac{1}{N} \mathbb{E}\left[\hat{\mathbf{Q}}_{1}\left(\hat{\mathbf{R}} \odot \mathbf{R}^{T} \odot \hat{\mathbf{Q}}_{1}\right) \Psi \phi\right] \\
& -\mathrm{j} u \sum_{m=1}^{L} \frac{1}{2 \pi \mathrm{j}} \oint_{\mathcal{C}^{-}} f_{m}\left(z_{2}\right) \frac{1+\varsigma}{N} \mathbb{E}\left[\hat{\mathbf{Q}}_{1} \operatorname{dg}\left(\mathbf{R} \hat{\mathbf{\Upsilon}}_{2} \hat{\mathbf{R}}\right) \Psi \phi\right] d z_{2} \\
& +\mathcal{O}\left(N^{-\mathbb{N}}\right) .
\end{aligned}
$$

With these two equations, we have obtained an expression for the "other terms" in equations (60) and (61) above. As explained before, one can combine these two equations by using the identity in (62). After left multiplying the result by the deterministic matrix $\overline{\mathbf{Q}}_{1}$ we obtain the following fundamental equation:

$$
\begin{aligned}
& \mathbb{E}\left[\left(\hat{\mathbf{Q}}_{1}-\overline{\mathbf{Q}}_{1}\right) \Psi \phi\right] \\
& =-z_{1} \frac{1}{N} \mathbb{E}\left[\hat{\mathbf{Q}}_{1} \hat{\mathbf{R}} \operatorname{dg}\left(\mathbf{R} \hat{\mathbf{Q}}_{1}\right) \overline{\mathbf{Q}}_{1} \Psi \phi\right] \\
& -\varsigma z_{1} \frac{1}{N} \mathbb{E}\left[\hat{\mathbf{Q}}_{1} \mathbf{R} \operatorname{dg}\left(\hat{\mathbf{R}} \hat{\mathbf{Q}}_{1}\right) \overline{\mathbf{Q}}_{1} \Psi \phi\right] \\
& +\frac{z_{1}}{\omega_{1}} \frac{1}{N} \mathbb{E}\left[\hat{\mathbf{Q}}_{1} \hat{\mathbf{R}} \overline{\mathbf{Q}}_{1} \Psi \phi \beta_{1}\right]+\varsigma \frac{z_{1}}{\omega_{1}} \frac{1}{N} \mathbb{E}\left[\hat{\mathbf{Q}}_{1} \mathbf{R} \hat{\mathbf{Q}}_{1} \hat{\mathbf{R}} \overline{\mathbf{Q}}_{1} \Psi \phi\right] \\
& -\frac{z_{1}^{2}}{\omega_{1}} \frac{(1+\varsigma)}{N} \mathbb{E}\left[\hat{\mathbf{Q}}_{1} \operatorname{dg}\left(\hat{\mathbf{Q}}_{1} \mathbf{R}\right) \hat{\mathbf{R}} \overline{\mathbf{Q}}_{1} \Psi \phi\right] \\
& +z_{1}^{2} \frac{1+\varsigma}{N} \mathbb{E}\left[\hat{\mathbf{Q}}_{1}\left(\hat{\mathbf{R}} \odot \mathbf{R}^{T} \odot \hat{\mathbf{Q}}_{1}\right) \overline{\mathbf{Q}}_{1} \Psi \phi\right] \\
& +\mathrm{j} \frac{z_{1}}{\omega_{1}} u \sum_{m=1}^{L} \frac{1}{2 \pi \mathrm{j}} \oint_{\mathcal{C}^{-}} f_{m}\left(z_{2}\right) \frac{1+\varsigma}{N} \times \\
& \times \mathbb{E}\left[\hat{\mathbf{Q}}_{1}\left[\mathbf{R} \hat{\boldsymbol{\Upsilon}}_{2} \hat{\mathbf{R}}-\omega_{1} \operatorname{dg}\left(\mathbf{R} \hat{\boldsymbol{\Upsilon}}_{2} \hat{\mathbf{R}}\right)\right] \overline{\mathbf{Q}}_{1} \Psi \phi\right] d z_{2}+\mathcal{O}\left(N^{-\mathbb{N}}\right) .
\end{aligned}
$$

We could try to use this expression on the first term on the right hand side of (72) and, after taking the traces, obtain the expression for $\mathbb{E}\left[\alpha_{1} \Psi \phi\right]$ that we are looking for. However, it turns out that the terms in $\beta_{1}$ will need to be further developed. For this reason, we will first consider the fundamental equation in (73) as a means to obtain an expression for $\mathbb{E}\left[\beta_{1} \Psi \phi\right]$, which will prove to be useful in the further development.

In order to obtain an expression for $\mathbb{E}\left[\beta_{1} \Psi \phi\right]$, consider again the equality in (73). Multiplying both sides by $\mathbf{R}$, taking traces and recalling the definition of $\beta_{1}$ in (58), we see that

$$
\begin{aligned}
& \left(1-\Gamma_{11}\right) \mathbb{E}\left[\beta_{1} \Psi \phi\right] \\
& =-z_{1} \frac{1}{N} \mathbb{E} \operatorname{tr}\left[\hat{\mathbf{Q}}_{1} \hat{\mathbf{R}} \operatorname{dg}\left(\mathbf{R} \hat{\mathbf{Q}}_{1}\right) \overline{\mathbf{Q}}_{1} \mathbf{R} \Psi \phi\right] \\
& -\varsigma z_{1} \frac{1}{N} \mathbb{E} \operatorname{tr}\left[\hat{\mathbf{Q}}_{1} \mathbf{R} \operatorname{dg}\left(\hat{\mathbf{R}} \hat{\mathbf{Q}}_{1}\right) \overline{\mathbf{Q}}_{1} \mathbf{R} \Psi \phi\right] \\
& +\frac{z_{1}}{\omega_{1}} \frac{1}{N} \mathbb{E}\left[\rho_{1} \beta_{1} \Psi \phi\right]+\varsigma \frac{z_{1}}{\omega_{1}} \frac{1}{N} \mathbb{E} \operatorname{tr}\left[\hat{\mathbf{Q}}_{1} \mathbf{R} \hat{\mathbf{Q}}_{1} \hat{\mathbf{R}} \overline{\mathbf{Q}}_{1} \mathbf{R} \Psi \phi\right] \\
& -\frac{z_{1}^{2}}{\omega_{1}} \frac{1+\varsigma}{N} \mathbb{E} \operatorname{tr}\left[\hat{\mathbf{Q}}_{1} \operatorname{dg}\left(\hat{\mathbf{Q}}_{1} \mathbf{R}\right) \hat{\mathbf{R}} \overline{\mathbf{Q}}_{1} \mathbf{R} \Psi \phi\right] \\
& +z_{1}^{2} \frac{1+\varsigma}{N} \mathbb{E} \operatorname{tr}\left[\left(\hat{\mathbf{R}} \odot \hat{\mathbf{Q}}_{1}\right)\left(\overline{\mathbf{Q}}_{1} \mathbf{R} \hat{\mathbf{Q}}_{1} \odot \mathbf{R}\right) \Psi \phi\right] \\
& +\mathrm{j} \frac{z_{1}}{\omega_{1}} u \sum_{m=1}^{L} \frac{1}{2 \pi \mathrm{j}} \oint_{\mathcal{C}^{-}} f_{m}\left(z_{2}\right) \frac{1+\varsigma}{N} \times \\
& \times \mathbb{E} \operatorname{tr}\left[\hat{\mathbf{Q}}_{1}\left[\mathbf{R} \hat{\mathbf{\Upsilon}}_{2} \hat{\mathbf{R}}-\omega_{1} \operatorname{dg}\left(\mathbf{R} \hat{\mathbf{\Upsilon}}_{2} \hat{\mathbf{R}}\right)\right] \overline{\mathbf{Q}}_{1} \mathbf{R} \Psi \phi\right] d z_{2}+\mathcal{O}\left(N^{-\mathbb{N}}\right)
\end{aligned}
$$

where we have defined

$$
\rho_{1}=\operatorname{tr}\left[\hat{\mathbf{Q}}_{1} \hat{\mathbf{R}} \overline{\mathbf{Q}}_{1} \mathbf{R} \phi\right]-\frac{z_{1}}{\omega_{1}} \operatorname{tr}\left[\overline{\mathbf{Q}}_{1} \mathbf{R} \overline{\mathbf{Q}}_{1} \mathbf{R}\right]
$$

and also $\Gamma_{11}=\Gamma_{M}\left(\omega_{M}\left(z_{1}\right), \omega_{M}\left(z_{1}\right)\right)$, where

$$
\Gamma_{M}\left(\omega_{1}, \omega_{2}\right)=\frac{1}{N} \sum_{m=1}^{\bar{M}} K_{m} \frac{\gamma_{m}^{2}}{\left(\gamma_{m}-\omega_{1}\right)\left(\gamma_{m}-\omega_{2}\right)} .
$$


It can be shown (see Appendix F) that

$$
\sup _{M} \sup _{\left(\omega_{1}, \omega_{2}\right) \in \mathcal{C}_{\omega} \times \mathcal{C}_{\omega}}\left|\Gamma_{M}\left(\omega_{1}, \omega_{2}\right)\right|<1
$$

and therefore we can divide both sides of (74) by $1-\Gamma_{11}$ in order to obtain an expression for $\mathbb{E}\left[\beta_{1} \Psi \phi\right]$. Using this expression in (73), multiplying both sides by $\mathbf{D}$ and inserting the result into (72), we finally obtain an expression for $\mathbb{E}\left[\alpha_{1} \Psi \phi\right]$, given by

$$
\begin{aligned}
\mathbb{E}\left[\alpha_{1} \Psi \phi\right] & =\tilde{\mu}\left(z_{1}\right)+\mathrm{j} u \sum_{m=1}^{L} \frac{1}{2 \pi \mathrm{j}} \oint_{\mathcal{C}^{-}} \tilde{\Theta}\left(z_{1}, z_{2}\right) f_{m}\left(z_{2}\right) d z_{2} \\
& +\tilde{\xi}\left(z_{1}\right)+\mathcal{O}\left(N^{-\mathbb{N}}\right)
\end{aligned}
$$

where $\tilde{\mu}\left(z_{1}\right), \tilde{\Theta}\left(z_{1}, z_{2}\right)$ and $\tilde{\xi}\left(z_{1}\right)$ are defined as follows. The term $\tilde{\mu}\left(z_{1}\right)$ contains the information about the asymptotic mean and can be derived into two parts, namely $\tilde{\mu}\left(z_{1}\right)=$ $\tilde{\mu}_{1}\left(z_{1}\right)+\tilde{\mu}_{2}\left(z_{1}\right)$, where the first term

$$
\begin{aligned}
\tilde{\mu}_{1}\left(z_{1}\right) & =-\varsigma \frac{1}{\omega_{1}} \frac{1}{N} \mathbb{E} \operatorname{tr}\left[\hat{\mathbf{R}} \hat{\mathbf{Q}}_{1} \mathbf{R} \hat{\mathbf{Q}}_{1} \Psi \phi\right] \\
& +\varsigma \frac{1}{\omega_{1}}\left(\frac{z_{1}}{\omega_{1}}\right)^{2} \frac{1}{1-\Gamma_{11}} \frac{1}{N} \mathbb{E} \operatorname{tr}\left[\mathbf{R} \hat{\mathbf{Q}}_{1} \mathbf{R} \hat{\mathbf{Q}}_{1} \hat{\mathbf{R}} \overline{\mathbf{Q}}_{1} \Psi \phi\right]
\end{aligned}
$$

appears only if the observations are real-valued, whereas the second one -given by (79) at the top of next page- is always present. The term $\tilde{\Theta}\left(z_{1}, z_{2}\right)$ is associated with the asymptotic covariance, and can be expressed as

$$
\tilde{\Theta}\left(z_{1}, z_{2}\right)=\left(\frac{z_{1}}{\omega_{1}}\right)^{2} \frac{1}{1-\Gamma_{11}} \frac{1+\varsigma}{N} \mathbb{E} \operatorname{tr}\left[\hat{\mathbf{R}} \hat{\Upsilon}_{1} \mathbf{R} \hat{\Upsilon}_{2} \Psi \phi\right]
$$

$$
+\frac{1}{\omega_{1}}\left(\frac{z_{1}}{\omega_{1}} \frac{1}{1-\Gamma_{11}}-1\right) \frac{1+\varsigma}{N} \mathbb{E} \operatorname{tr}\left[\hat{\mathbf{R}} \hat{\mathbf{Q}}_{1} \mathbf{R} \hat{\Upsilon}_{2} \Psi \phi\right]
$$

where we have defined the matrix

$$
\hat{\mathbf{\Upsilon}}_{1}=\overline{\mathbf{Q}}_{1} \mathbf{D} \hat{\mathbf{Q}}_{1}-\operatorname{dg}\left(\overline{\mathbf{Q}}_{1} \mathbf{R} \hat{\mathbf{Q}}_{1}\right)
$$

and where $\hat{\Upsilon}_{2}$ is defined in (66). Finally the term $\tilde{\xi}\left(z_{1}\right)$ is a residual error that takes the form

$$
\begin{aligned}
\tilde{\xi}\left(z_{1}\right) & =-\frac{z_{1}}{\omega_{1}} \frac{1}{N} \mathbb{E}\left[\alpha_{1} \beta_{1} \Psi \phi\right] \\
& +\frac{1}{\omega_{1}}\left(\frac{z_{1}}{\omega_{1}}\right)^{2} \frac{1}{1-\Gamma_{11}} \frac{1}{N} \mathbb{E}\left[\beta_{1} \rho_{1} \Psi \phi\right] .
\end{aligned}
$$

Observe that the identity in (77) corresponds with the one in (63), where now the "other terms" have been fully established. By simple identification of the different terms in (77) with those in (64), we see that the proof will be concluded once we show that

$$
\begin{aligned}
\tilde{\mu}\left(z_{1}\right) & =\frac{\bar{\mu}\left(\omega_{1}\right)}{\mathcal{D}\left(\omega_{1}\right)} \mathbb{E}[\Psi]+\mathcal{O}\left(N^{-1}\right) \\
\tilde{\Theta}\left(z_{1}, z_{2}\right) & =\left(\frac{\bar{\sigma}^{2}\left(\omega_{1}, \omega_{2}\right)}{\mathcal{D}\left(\omega_{1}\right) \mathcal{D}\left(\omega_{2}\right)}+h\left(z_{1}, z_{2}\right)\right) \mathbb{E}[\Psi]+\mathcal{O}\left(N^{-1}\right) \\
\tilde{\xi}\left(z_{1}\right) & =\mathcal{O}\left(N^{-1}\right)
\end{aligned}
$$

where $\bar{\mu}\left(\omega_{1}\right), \bar{\sigma}^{2}\left(\omega_{1}, \omega_{2}\right)$ and $\mathcal{D}(\omega)$ are as defined in (35), (37) and (20) respectively (dropping the dependence on $M$ ) and where $h\left(z_{1}, z_{2}\right)$ denotes any bivariate complex function such that

$$
\frac{-1}{4 \pi^{2}} \oint_{\mathcal{C}^{-}} \oint_{\mathcal{C}^{-}} h\left(z_{1}, z_{2}\right) d z_{1} d z_{2}=0
$$

so that this term does not contribute to the asymptotic variance.

We will begin by analyzing the error term $\tilde{\xi}\left(z_{1}\right)$, and then proceed to the study of the mean and covariance related quantities $\tilde{\mu}\left(z_{1}\right)$ and $\tilde{\Theta}\left(z_{1}, z_{2}\right)$.

\section{Analysis of the error term $\tilde{\xi}\left(z_{1}\right)$}

The objective of this subsection is to prove that the error term $\tilde{\xi}\left(z_{1}\right)$ decays as in (84). By applying the triangular inequality in the definition of $\tilde{\xi}\left(z_{1}\right)$ in (81) and using the bounds in (70) and (76), it suffices to investigate the terms $N^{-1} \mathbb{E}\left[\alpha_{1} \beta_{1} \Psi \phi\right]$ and $N^{-1} \mathbb{E}\left[\beta_{1} \rho_{1} \Psi \phi\right]$. A direct application of the Cauchy-Schwarz inequality leads to

$$
\begin{aligned}
\left|N^{-1} \mathbb{E}\left[\alpha_{1} \beta_{1} \Psi \phi\right]\right|^{2} \leq \mathbb{E}\left[\frac{\left|\alpha_{1}\right|^{2}}{N} \phi\right] \mathbb{E}\left[\frac{\left|\beta_{1}\right|^{2}}{N} \phi\right] \\
=N^{2}\left(\left|\mathbb{E} \frac{\alpha_{1}}{N}\right|^{2}+\operatorname{var}\left(\frac{\alpha_{1}}{N}\right)\right)\left(\left|\mathbb{E} \frac{\beta_{1}}{N}\right|^{2}+\operatorname{var}\left(\frac{\beta_{1}}{N}\right)\right) \\
+\mathcal{O}\left(N^{-\mathbb{N}}\right) .
\end{aligned}
$$

The following proposition leads to the desired result.

Proposition 3: Assume that $\mathbf{A}_{i}, i=1,2,3$ are deterministic matrices with bounded spectral norm, and let $\mathbf{B}_{1}$ be an Hermitian positive semidefinite matrix with bounded entries. Consider the following two functions

$$
\begin{aligned}
& \digamma_{1}\left(z_{1}\right)=\frac{1}{N} \operatorname{tr}\left[\left(\mathbf{A}_{1} \hat{\mathbf{Q}}_{1} \mathbf{A}_{2} \odot \mathbf{B}_{1}\right) \mathbf{A}_{3} \phi\right] \\
& \widetilde{\digamma}_{1}\left(z_{1}\right)=\frac{1}{N} \operatorname{tr}\left[\left(\mathbf{A}_{1} \hat{\mathbf{Q}}_{1} \hat{\mathbf{R}} \mathbf{A}_{2} \odot \mathbf{B}_{1}\right) \mathbf{A}_{3} \phi\right]
\end{aligned}
$$

Then,

$$
\mathbb{E}\left[\digamma_{1}\left(z_{1}\right)\right]=\frac{1}{N} \operatorname{tr}\left[\left(\mathbf{A}_{1} \overline{\mathbf{Q}}_{1} \mathbf{A}_{2} \odot \mathbf{B}_{1}\right) \mathbf{A}_{3}\right]+\mathcal{O}\left(N^{-1}\right)
$$

$\mathbb{E}\left[\widetilde{\boldsymbol{\digamma}}_{1}\left(z_{1}\right)\right]=\frac{z_{1}}{\omega_{1}} \frac{1}{N} \operatorname{tr}\left[\left(\mathbf{A}_{1} \overline{\mathbf{Q}}_{1} \mathbf{R} \mathbf{A}_{2} \odot \mathbf{B}_{1}\right) \mathbf{A}_{3}\right]+\mathcal{O}\left(N^{-1}\right)$

Furthermore, $\operatorname{var} \digamma_{1}\left(z_{1}\right)=\mathcal{O}\left(N^{-2}\right)$ and $\operatorname{var} \widetilde{\digamma}_{1}\left(z_{1}\right)=$ $\mathcal{O}\left(N^{-2}\right)$

Proof: The result follows from the application of Proposition 2, see further Appendix D.

Direct application of this proposition shows that $\left|N^{-1} \mathbb{E} \alpha_{1}\right|^{2}=\mathcal{O}\left(N^{-2}\right)$ and $\operatorname{var}\left(N^{-1} \alpha_{1}\right)=\mathcal{O}\left(N^{-2}\right)$, and that the same holds if $\alpha_{1}$ is replaced by $\beta_{1}$. Inserting the result into (86), we have shown that $N^{-1} \mathbb{E}\left[\alpha_{1} \beta_{1} \Psi \phi\right]=\mathcal{O}\left(N^{-1}\right)$. Proceeding in the same way, we can also established that $N^{-1} \mathbb{E}\left[\beta_{1} \rho_{1} \Psi \phi\right]=\mathcal{O}\left(N^{-1}\right)$, which directly leads to $\tilde{\xi}\left(z_{1}\right)=\mathcal{O}\left(N^{-1}\right)$ as we wanted to show. 


$$
\begin{aligned}
\tilde{\mu}_{2}\left(z_{1}\right) & =\frac{z_{1}}{\omega_{1}} \frac{1+\varsigma}{N} \mathbb{E} \operatorname{tr}\left[\hat{\mathbf{R}} \hat{\mathbf{Q}}_{1} \operatorname{dg}\left(\hat{\mathbf{Q}}_{1} \mathbf{R}\right) \Psi \phi\right]-\frac{\left(z_{1} / \omega_{1}\right)^{2}}{1-\Gamma_{11}} \frac{1}{N} \mathbb{E} \operatorname{tr}\left[\mathbf{R} \hat{\mathbf{Q}}_{1}\left[\hat{\mathbf{R}} \operatorname{dg}\left(\mathbf{R} \hat{\mathbf{Q}}_{1}\right)+\varsigma \mathbf{R} \operatorname{dg}\left(\hat{\mathbf{R}} \hat{\mathbf{Q}}_{1}\right)\right] \overline{\mathbf{Q}}_{1} \Psi \phi\right] \\
& -\frac{\left(z_{1} / \omega_{1}\right)^{3}}{1-\Gamma_{11}} \frac{1+\varsigma}{N} \mathbb{E} \operatorname{tr}\left[\mathbf{R} \hat{\mathbf{Q}}_{1} \operatorname{dg}\left(\hat{\mathbf{Q}}_{1} \mathbf{R}\right) \hat{\mathbf{R}} \overline{\mathbf{Q}}_{1} \Psi \phi\right]+z_{1} \frac{\left(z_{1} / \omega_{1}\right)^{2}}{1-\Gamma_{11}} \frac{1+\varsigma}{N} \mathbb{E} \operatorname{tr}\left[\left(\hat{\mathbf{R}} \odot \hat{\mathbf{Q}}_{1}\right)\left(\overline{\mathbf{Q}}_{1} \mathbf{R} \hat{\mathbf{Q}}_{1} \odot \mathbf{R}\right) \Psi \phi\right]
\end{aligned}
$$

\section{Analysis of the mean-related term $\tilde{\mu}\left(z_{1}\right)$}

The procedure that we follow in order to analyze the different terms of $\tilde{\mu}\left(z_{1}\right)$ is as follows. We observe that $\tilde{\mu}\left(z_{1}\right)$ can be expressed as the sum of quantities of the type $\mathbb{E}[X \Psi \phi]$ where $X$ is a certain random variable, typically expressed as the normalized trace of a function of the sample correlation matrix. The idea here is to decorrelate this random variable from the characteristic function, by expressing

$$
\mathbb{E}[X \Psi \phi]=\mathbb{E}[X \phi] \mathbb{E}[\Psi]+\mathbb{E}\left[(X \phi)^{\circ} \Psi\right]
$$

where $(\cdot)^{\circ} \doteq(\cdot)-\mathbb{E}(\cdot)$. The second term above can be bounded by virtue of the Cauchy-Schwarz inequality, namely

$$
\left|\mathbb{E}\left[(X \phi)^{\circ} \Psi\right]\right| \leq \sqrt{\operatorname{var}(X \phi) \mathbb{E}\left[|\Psi|^{2}\right]}=\sqrt{\operatorname{var}(X \phi)}
$$

where we used the fact that $|\Psi|=1$. By showing that $\mathbb{E}[X \phi]=\bar{X}+\mathcal{O}\left(N^{-1}\right)$ for a certain deterministic quantity $\bar{X}$, together with $\operatorname{var}(X \phi)=\mathcal{O}\left(N^{-2}\right)$, it readily follows that $\mathbb{E}[X \Psi \phi]=\bar{X}+\mathcal{O}\left(N^{-1}\right)$. We will apply this reasoning to each of the terms that define $\tilde{\mu}\left(z_{1}\right)$, and this will lead us to the desired result.

As before, we divide $\tilde{\mu}\left(z_{1}\right)$ into two terms, $\tilde{\mu}\left(z_{1}\right)=$ $\tilde{\mu}_{1}\left(z_{1}\right)+\tilde{\mu}_{2}\left(z_{1}\right)$, and analyze them separately. Let us begin with the quantity $\tilde{\mu}_{1}\left(z_{1}\right)$ in (78), which is only different from zero if the observations are real valued. Using the bounds in (70) and (76), we only need to study the asymptotic behavior of quantities $N^{-1} \mathbb{E} \operatorname{tr}\left[\hat{\mathbf{Q}}_{1} \mathbf{R} \hat{\mathbf{Q}}_{1} \hat{\mathbf{R}} \Psi \phi\right]$ and $N^{-1} \mathbb{E} \operatorname{tr}\left[\mathbf{R} \hat{\mathbf{Q}}_{1} \mathbf{R} \hat{\mathbf{Q}}_{1} \hat{\mathbf{R}} \overline{\mathbf{Q}}_{1} \Psi \phi\right]$. We will use the following result, which can be established using Proposition 2.

Proposition 4: Assume that $\mathbf{A}_{i}, i=1,2,3,4$ are deterministic $M \times M$ matrices with bounded spectral norm, and let $\mathbf{B}_{1}$ and $\mathbf{B}_{2}$ denote two $M \times M$ Hermitian positive semi-definite matrices with bounded entries. Define the two functions $\digamma_{2}=\digamma_{2}\left(z_{1}, z_{2}\right)$ and $\widetilde{\digamma}_{2}=\widetilde{\digamma}_{2}\left(z_{1}, z_{2}\right)$ as

$\digamma_{2}=\frac{1}{N} \operatorname{tr}\left[\mathbf{A}_{1}\left(\mathbf{A}_{2} \hat{\mathbf{Q}}_{1} \mathbf{A}_{3} \odot \mathbf{B}_{1}\right) \mathbf{A}_{4}\left(\mathbf{A}_{5} \hat{\mathbf{Q}}_{2} \mathbf{A}_{6} \odot \mathbf{B}_{2}\right) \phi\right]$

$\widetilde{\digamma}_{2}=\frac{1}{N} \operatorname{tr}\left[\mathbf{A}_{1}\left(\mathbf{A}_{2} \hat{\mathbf{Q}}_{1} \hat{\mathbf{R}} \mathbf{A}_{3} \odot \mathbf{B}_{1}\right) \mathbf{A}_{4}\left(\mathbf{A}_{5} \hat{\mathbf{Q}}_{2} \mathbf{A}_{6} \odot \mathbf{B}_{2}\right) \phi\right]$

We can state that $\mathbb{E}\left[\digamma_{2}\right]$ and $\mathbb{E}\left[\widetilde{\digamma}_{2}\right]$ can be expressed as in (95)-(96) at the top of next page. Furthermore, $\operatorname{var} \digamma_{2}\left(z_{1}, z_{2}\right)=\mathcal{O}\left(N^{-2}\right)$ and $\operatorname{var} \widetilde{\digamma}_{2}\left(z_{1}, z_{2}\right)=\mathcal{O}\left(N^{-2}\right)$.

Proof: See Appendix D.

Proposition 4 provides a very general result but may turn out to be difficult to manage in the characterization of $\tilde{\mu}\left(z_{1}\right)$. For this reason, we provide next a more particular result that will be more convenient in order to study the two quantities in this section.
Corollary 2: Assume that $\mathbf{A}_{i}, i=1,2$ are deterministic $M \times M$ matrices with bounded spectral norm. Then, we can write

$$
\begin{array}{r}
\frac{1}{N} \mathbb{E} \operatorname{tr}\left[\mathbf{A}_{1} \hat{\mathbf{Q}}_{1} \hat{\mathbf{R}} \mathbf{A}_{2} \hat{\mathbf{Q}}_{2} \phi\right]=\frac{z_{1}}{\omega_{1}} \frac{1}{N} \mathbb{E} \operatorname{tr}\left[\mathbf{A}_{1} \overline{\mathbf{Q}}_{1} \mathbf{R} \mathbf{A}_{2} \overline{\mathbf{Q}}_{2}\right] \\
-\frac{z_{1}}{\omega_{1}} \frac{z_{2}}{\omega_{2}} \frac{1}{1-\Gamma_{12}} \frac{1}{N} \mathbb{E} \operatorname{tr}\left[\mathbf{A}_{1} \overline{\mathbf{Q}}_{1} \mathbf{R} \overline{\mathbf{Q}}_{2}\right] \frac{1}{N} \operatorname{tr}\left[\overline{\mathbf{Q}}_{2} \mathbf{R} \mathbf{A}_{2}\right] \\
+\frac{\left(z_{1} / \omega_{1}\right)^{2}\left(z_{2} / \omega_{2}\right)}{1-\Gamma_{12}} \frac{1}{N} \mathbb{E} \operatorname{tr}\left[\mathbf{A}_{1} \overline{\mathbf{Q}}_{1} \mathbf{R} \overline{\mathbf{Q}}_{2}\right] \frac{1}{N} \operatorname{tr}\left[\mathbf{R} \overline{\mathbf{Q}}_{1} \mathbf{R} \mathbf{A}_{2} \overline{\mathbf{Q}}_{2}\right] \\
+\mathcal{O}\left(N^{-1}\right)
\end{array}
$$

and

$$
\begin{aligned}
\frac{1}{N} \mathbb{E} \operatorname{tr}\left[\mathbf{A}_{1} \hat{\mathbf{Q}}_{1} \mathbf{A}_{2} \hat{\mathbf{Q}}_{2} \phi\right]=\frac{1}{N} \mathbb{E} \operatorname{tr}\left[\mathbf{A}_{1} \overline{\mathbf{Q}}_{1} \mathbf{A}_{2} \overline{\mathbf{Q}}_{2}\right] \\
+\frac{\left(z_{1} / \omega_{1}\right)\left(z_{2} / \omega_{2}\right)}{1-\Gamma_{12}} \frac{1}{N} \mathbb{E} \operatorname{tr}\left[\mathbf{A}_{1} \overline{\mathbf{Q}}_{1} \mathbf{R} \overline{\mathbf{Q}}_{2}\right] \frac{1}{N} \operatorname{tr}\left[\mathbf{A}_{2} \overline{\mathbf{Q}}_{2} \mathbf{R} \overline{\mathbf{Q}}_{1}\right] \\
+\mathcal{O}\left(N^{-1}\right)
\end{aligned}
$$

where $\Gamma_{12}$ is defined in (75). Furthermore, the variance of these two quantities can be written as $\mathcal{O}\left(N^{-2}\right)$.

Proof: Particularizing Proposition 4 to the case where $\mathbf{B}_{1}=\mathbf{B}_{2}=\mathbf{1 1}^{T}$, where $\mathbf{1}$ is an all-ones column vector, and using the fact that $\mathbf{1}^{T}\left(\mathbf{A}_{1} \odot \mathbf{A}_{2}^{T}\right) \mathbf{1}=\operatorname{tr}\left(\mathbf{A}_{1} \mathbf{A}_{2}\right)$ we see that

$$
\begin{gathered}
\frac{1}{N} \mathbb{E} \operatorname{tr}\left[\mathbf{A}_{1} \hat{\mathbf{Q}}_{1} \hat{\mathbf{R}} \mathbf{A}_{2} \hat{\mathbf{Q}}_{2} \phi\right]=\frac{z_{1}}{\omega_{1}} \frac{1}{N} \mathbb{E} \operatorname{tr}\left[\mathbf{A}_{1} \overline{\mathbf{Q}}_{1} \mathbf{R} \mathbf{A}_{2} \overline{\mathbf{Q}}_{2}\right] \\
+\frac{z_{1}^{2}}{\omega_{1}} \frac{z_{2}}{\omega_{2}} \frac{1}{N} \mathbb{E} \operatorname{tr}\left[\mathbf{A}_{1} \hat{\mathbf{Q}}_{1} \mathbf{R} \hat{\mathbf{Q}}_{2} \phi\right] \frac{1}{N} \operatorname{tr}\left[\overline{\mathbf{Q}}_{2} \mathbf{R} \overline{\mathbf{Q}}_{1} \mathbf{D} \mathbf{A}_{2}\right]+\mathcal{O}\left(N^{-1}\right)
\end{gathered}
$$

and also

$$
\begin{aligned}
& \frac{1}{N} \mathbb{E} \operatorname{tr}\left[\mathbf{A}_{1} \hat{\mathbf{Q}}_{1} \mathbf{A}_{2} \hat{\mathbf{Q}}_{2} \phi\right]=\frac{1}{N} \mathbb{E} \operatorname{tr}\left[\mathbf{A}_{1} \overline{\mathbf{Q}}_{1} \mathbf{A}_{2} \overline{\mathbf{Q}}_{2}\right] \\
& +\frac{z_{1}}{\omega_{1}} \frac{z_{2}}{\omega_{2}} \frac{1}{N} \mathbb{E} \operatorname{tr}\left[\mathbf{A}_{1} \hat{\mathbf{Q}}_{1} \mathbf{R} \hat{\mathbf{Q}}_{2} \phi\right] \frac{1}{N} \operatorname{tr}\left(\overline{\mathbf{Q}}_{2} \mathbf{R} \overline{\mathbf{Q}}_{1} \mathbf{A}_{2}\right)+\mathcal{O}\left(N^{-1}\right) .
\end{aligned}
$$

where we have additionally used Proposition 3 together with the bounds in Lemma 1. Particularizing the second equation to the case $\mathbf{A}_{2}=\mathbf{R}$ we obtain

$$
\begin{aligned}
\frac{1}{N} \mathbb{E} \operatorname{tr}\left[\mathbf{A}_{1} \hat{\mathbf{Q}}_{1} \mathbf{R} \hat{\mathbf{Q}}_{2} \phi\right] & =\frac{1}{1-\Gamma_{12}} \frac{1}{N} \mathbb{E} \operatorname{tr}\left[\mathbf{A}_{1} \overline{\mathbf{Q}}_{1} \mathbf{R} \overline{\mathbf{Q}}_{2}\right] \\
& +\mathcal{O}\left(N^{-1}\right)
\end{aligned}
$$

where we have implicitly used the bound in (76). Replacing this result into the first two equations, we obtain the result.

We have now all the ingredients to characterize the asymptotic behavior of the mean and variances of $N^{-1} \operatorname{tr}\left[\hat{\mathbf{Q}}_{1} \mathbf{R} \hat{\mathbf{Q}}_{1} \hat{\mathbf{R}} \phi\right]$ and $N^{-1} \operatorname{tr}\left[\mathbf{R} \hat{\mathbf{Q}}_{1} \mathbf{R} \hat{\mathbf{Q}}_{1} \hat{\mathbf{R}} \overline{\mathbf{Q}}_{1} \phi\right]$ that appear in (78). Using the fact that the variances of these two 


$$
\begin{aligned}
\mathbb{E}\left[\digamma_{2}\left(z_{1}, z_{2}\right)\right]=\frac{1}{N} & \operatorname{tr}\left[\mathbf{A}_{1}\left(\mathbf{A}_{2} \overline{\mathbf{Q}}_{1} \mathbf{A}_{3} \odot \mathbf{B}_{1}\right) \mathbf{A}_{4}\left(\mathbf{A}_{5} \overline{\mathbf{Q}}_{2} \mathbf{A}_{6} \odot \mathbf{B}_{2}\right)\right] \\
& +\frac{z_{1}}{\omega_{1}} \frac{1}{N^{2}} \mathbb{E} \operatorname{tr}\left[\left(\mathbf{A}_{1} \odot\left(\mathbf{A}_{2} \hat{\mathbf{Q}}_{1} \mathbf{R} \hat{\mathbf{Q}}_{2} \mathbf{A}_{6}\right)^{T}\right) \mathbf{B}_{1}\left(\mathbf{A}_{4} \odot\left(\mathbf{A}_{5} \hat{\mathbf{Q}_{2}} \hat{\mathbf{R}} \overline{\mathbf{Q}}_{1} \mathbf{A}_{3}\right)^{T}\right) \mathbf{B}_{2} \phi\right]+\mathcal{O}\left(N^{-1}\right) .
\end{aligned}
$$

$$
\begin{aligned}
\mathbb{E}\left[\widetilde{\digamma}_{2}\left(z_{1}, z_{2}\right)\right]= & \frac{z_{1}}{\omega_{1}} \frac{1}{N} \operatorname{tr}\left[\mathbf{A}_{1}\left(\mathbf{A}_{2} \overline{\mathbf{Q}}_{1} \mathbf{R} \mathbf{A}_{3} \odot \mathbf{B}_{1}\right) \mathbf{A}_{4}\left(\mathbf{A}_{5} \overline{\mathbf{Q}}_{2} \mathbf{A}_{6} \odot \mathbf{B}_{2}\right)\right] \\
& +\frac{z_{1}^{2}}{\omega_{1}} \frac{1}{N^{2}} \mathbb{E} \operatorname{tr}\left[\left(\mathbf{A}_{1} \odot\left(\mathbf{A}_{2} \hat{\mathbf{Q}}_{1} \mathbf{R} \hat{\mathbf{Q}}_{2} \mathbf{A}_{6}\right)^{T}\right) \mathbf{B}_{1}\left(\mathbf{A}_{4} \odot\left(\mathbf{A}_{5} \hat{\mathbf{Q}}_{2} \hat{\mathbf{R}} \overline{\mathbf{Q}}_{1} \mathbf{D} \mathbf{A}_{3}\right)^{T}\right) \mathbf{B}_{2} \phi\right]+\mathcal{O}\left(N^{-1}\right)
\end{aligned}
$$

terms take the form $\mathcal{O}\left(N^{-2}\right)$ together with the reasoning in (91)-(92), we can write the term $\tilde{\mu}_{1}\left(z_{1}\right)$ as

$$
\begin{aligned}
\tilde{\mu}_{1}\left(z_{1}\right)=-\varsigma \frac{1}{\omega_{1}} \frac{1}{N} \mathbb{E} \operatorname{tr}\left[\mathbf{R} \hat{\mathbf{Q}}_{1} \hat{\mathbf{R}} \hat{\mathbf{Q}}_{1} \phi\right] \mathbb{E}[\Psi] \\
+\varsigma \frac{1}{\omega_{1}}\left(\frac{z_{1}}{\omega_{1}}\right)^{2} \frac{1}{1-\Gamma_{11}} \times \\
\times \frac{1}{N} \mathbb{E} \operatorname{tr}\left[\mathbf{R} \hat{\mathbf{Q}}_{1} \hat{\mathbf{R}} \overline{\mathbf{Q}}_{1} \mathbf{R} \hat{\mathbf{Q}}_{1} \phi\right] \mathbb{E}[\Psi]+\mathcal{O}\left(N^{-1}\right) .
\end{aligned}
$$

Therefore, developing the expectation of the two terms $N^{-1} \operatorname{tr}\left[\hat{\mathbf{Q}}_{1} \mathbf{R} \hat{\mathbf{Q}}_{1} \hat{\mathbf{R}} \phi\right]$ and $N^{-1} \operatorname{tr}\left[\mathbf{R} \hat{\mathbf{Q}}_{1} \mathbf{R} \hat{\mathbf{Q}}_{1} \hat{\mathbf{R}} \overline{\mathbf{Q}}_{1} \phi\right]$ according to Corollary 2 , we obtain

$$
\begin{aligned}
& \tilde{\mu}_{1}\left(z_{1}\right)=\varsigma \frac{1}{\omega_{1}} \frac{1}{\left(1-\Gamma_{11}\right)^{2}} \times \\
& \times\left(\left(\frac{z_{1}}{\omega_{1}}\right)^{3} \frac{1}{N} \operatorname{tr}\left[\left(\mathbf{R} \overline{\mathbf{Q}}_{1}\right)^{3}\right]-\Gamma_{11}\right) \mathbb{E}[\Psi]+\mathcal{O}\left(N^{-1}\right) .
\end{aligned}
$$

Let us now deal with the term $\tilde{\mu}_{2}\left(z_{1}\right)$ in (79), which is composed of four separate terms that will be denoted as $\tilde{\mu}_{2}^{(1)}\left(z_{1}\right), \ldots, \tilde{\mu}_{2}^{(4)}\left(z_{1}\right)$. Here again, using the bounds in (70) and (76) we may investigate the asymptotic behavior of each of the sum terms in $\tilde{\mu}_{2}\left(z_{1}\right)$ separately. The first two terms can be directly handled using Proposition 4 and decorrelating with respect to the characteristic function as in (91)-(92), that is

$$
\begin{aligned}
\tilde{\mu}_{2}^{(1)}\left(z_{1}\right) & =(1+\varsigma)\left(\frac{z_{1}}{\omega_{1}}\right)^{2} \times \\
& \times\left(\frac{1}{N} \mathbb{E} \operatorname{tr}\left[\overline{\mathbf{Q}}_{1} \mathbf{R} \operatorname{dg}\left(\mathbf{R} \overline{\mathbf{Q}}_{1}\right)\right]\right) \mathbb{E}[\Psi]+\mathcal{O}\left(N^{-1}\right) \\
\tilde{\mu}_{2}^{(2)}\left(z_{1}\right) & =-\frac{1+\varsigma}{1-\Gamma_{11}}\left(\frac{z_{1}}{\omega_{1}}\right)^{3} \times \\
& \times \frac{1}{N} \mathbb{E} \operatorname{tr}\left[\left(\overline{\mathbf{Q}}_{1} \mathbf{R}\right)^{2} \operatorname{dg}\left(\mathbf{R} \overline{\mathbf{Q}}_{1}\right)\right] \mathbb{E}[\Psi]+\mathcal{O}\left(N^{-1}\right)
\end{aligned}
$$

For the other two terms, we need some additional results, that we summarize in the following proposition.

Proposition 5: Let $\mathbf{A}_{i}, i=1,2$ be two deterministic $M \times$
$M$ matrices with bounded spectral norm. Then, we can write

$$
\begin{gathered}
\frac{1}{N} \mathbb{E} \operatorname{tr}\left[\hat{\mathbf{Q}}_{1} \operatorname{dg}\left(\hat{\mathbf{Q}}_{1} \mathbf{R}\right) \hat{\mathbf{R}} \mathbf{A}_{1} \phi\right] \\
=\frac{1}{N} \mathbb{E} \operatorname{tr}\left[\hat{\mathbf{Q}}_{1} \operatorname{dg}\left(\hat{\mathbf{Q}}_{1} \mathbf{R}\right) \mathbf{R} \mathbf{A}_{1}\right] \\
-\mathbb{E}\left[\frac{1}{N} \operatorname{tr}\left[\hat{\mathbf{Q}}_{1} \hat{\mathbf{R}} \mathbf{A}_{1}\right] \frac{1}{N} \operatorname{tr}\left[\mathbf{R} \hat{\mathbf{Q}}_{1} \operatorname{dg}\left(\hat{\mathbf{Q}}_{1} \mathbf{R}\right)\right]\right]+\mathcal{O}\left(N^{-1}\right)
\end{gathered}
$$

and also

$$
\begin{aligned}
& \frac{1}{N} \mathbb{E} \operatorname{tr}\left[\left(\mathbf{A}_{1} \hat{\mathbf{Q}}_{1} \odot \mathbf{A}_{2}\right)\left(\hat{\mathbf{Q}}_{2} \odot \hat{\mathbf{R}}\right) \phi\right]= \\
& =\frac{1}{N} \mathbb{E} \operatorname{tr}\left[\left(\mathbf{A}_{1} \hat{\mathbf{Q}}_{1} \odot \mathbf{A}_{2}\right)\left(\hat{\mathbf{Q}}_{2} \odot \mathbf{R}\right)\right]+\mathcal{O}\left(N^{-1}\right) .
\end{aligned}
$$

Furthermore, the variances of these two quantities are $\mathcal{O}\left(N^{-2}\right)$.

Proof: See Appendix D.

Let us first analyze the third term $\tilde{\mu}_{2}^{(3)}\left(z_{1}\right)$. With the help of the above proposition together with the bounds (70) and (76) and decorrelating with respect to the characteristic function as in (91)-(92), we are able to write

$$
\begin{aligned}
\frac{1}{N} \mathbb{E} \operatorname{tr}\left[\mathbf{R} \hat{\mathbf{Q}}_{1} \operatorname{dg}\left(\hat{\mathbf{Q}}_{1} \mathbf{R}\right) \hat{\mathbf{R}} \overline{\mathbf{Q}}_{1} \Psi \phi\right] \\
=\frac{1}{N} \mathbb{E} \operatorname{tr}\left[\mathbf{R} \overline{\mathbf{Q}}_{1} \mathbf{R} \hat{\mathbf{Q}}_{1} \operatorname{dg}\left(\hat{\mathbf{Q}}_{1} \mathbf{R}\right)\right] \mathbb{E}[\Psi] \\
\quad-\mathbb{E}\left[\frac{1}{N} \operatorname{tr}\left[\hat{\mathbf{Q}}_{1} \hat{\mathbf{R}} \overline{\mathbf{Q}}_{1} \mathbf{R}\right] \times\right. \\
\left.\times \frac{1}{N} \operatorname{tr}\left[\mathbf{R} \hat{\mathbf{Q}}_{1} \operatorname{dg}\left(\hat{\mathbf{Q}}_{1} \mathbf{R}\right)\right]\right] \mathbb{E}[\Psi]+\mathcal{O}\left(N^{-1}\right)
\end{aligned}
$$

The first term on the right hand side of the above equation can be handled using Proposition 4. As for the second term, we can apply again the decorrelation procedure and express the expectation of the product of two variables as the product of expectations plus an error term. More specifically, if $X$ and $Y$ are two random variables, we can write

$$
\begin{aligned}
\mathbb{E}[X Y] & =\mathbb{E}[X] \mathbb{E}[Y]+\mathbb{E}\left[X^{\circ} Y\right] \\
\left|\mathbb{E}\left[X^{\circ} Y\right]\right| & \leq \sqrt{\operatorname{var} X} \sqrt{\mathbb{E}\left[|Y|^{2}\right]} .
\end{aligned}
$$

If $Y$ is bounded and the variance of $X$ is $\mathcal{O}\left(N^{-2}\right)$, we can conclude that $\mathbb{E}[X Y]=\mathbb{E}[X] \mathbb{E}[Y]+\mathcal{O}\left(N^{-1}\right)$. Using this, 
together with Propositions 3 and 4 we obtain

$$
\begin{aligned}
& \tilde{\mu}_{2}^{(3)}\left(z_{1}\right) \\
& =-\frac{\left(z_{1} / \omega_{1}\right)^{3}}{1-\Gamma_{11}} \frac{1+\varsigma}{N} \mathbb{E} \operatorname{tr}\left[\left(\mathbf{R} \overline{\mathbf{Q}}_{1}\right)^{2} \operatorname{dg}\left(\overline{\mathbf{Q}}_{1} \mathbf{R}\right)\right] \mathbb{E}[\Psi] \\
& +\frac{\Gamma_{11}\left(z_{1} / \omega_{1}\right)^{2}}{1-\Gamma_{11}} \frac{1+\varsigma}{N} \operatorname{tr}\left[\mathbf{R} \overline{\mathbf{Q}}_{1} \operatorname{dg}\left(\overline{\mathbf{Q}}_{1} \mathbf{R}\right)\right] \mathbb{E}[\Psi]+\mathcal{O}\left(N^{-1}\right) .
\end{aligned}
$$

Finally, for the fourth term, we may also apply Proposition 5 and decorrelate with respect to the characteristic function, so that

$$
\begin{aligned}
& \frac{1}{N} \mathbb{E} \operatorname{tr}\left[\left(\hat{\mathbf{R}} \odot \hat{\mathbf{Q}}_{1}\right)\left(\overline{\mathbf{Q}}_{1} \mathbf{R} \hat{\mathbf{Q}}_{1} \odot \mathbf{R}\right) \Psi \phi\right] \\
& =\frac{1}{N} \mathbb{E} \operatorname{tr}\left[\left(\mathbf{R} \odot \hat{\mathbf{Q}}_{1}\right)\left(\overline{\mathbf{Q}}_{1} \mathbf{R} \hat{\mathbf{Q}}_{1} \odot \mathbf{R}\right) \phi\right] \mathbb{E}[\Psi]+\mathcal{O}\left(N^{-1}\right)
\end{aligned}
$$

Then, a direct application of Proposition 4 leads to

$$
\begin{aligned}
& \tilde{\mu}_{2}^{(4)}\left(z_{1}\right)=z_{1} \frac{1}{1-\Gamma_{11}}\left(\frac{z_{1}}{\omega_{1}}\right)^{2} \times \\
& \times \frac{1+\varsigma}{N} \operatorname{tr}\left[\left(\overline{\mathbf{Q}}_{1} \odot \mathbf{R}\right)\left(\overline{\mathbf{Q}}_{1} \mathbf{R} \overline{\mathbf{Q}}_{1} \odot \mathbf{R}\right)\right] \mathbb{E}[\Psi]+\mathcal{O}\left(N^{-1}\right) .
\end{aligned}
$$

Gathering the expression for $\tilde{\mu}_{1}\left(z_{1}\right)$ and $\tilde{\mu}_{2}^{(i)}\left(z_{1}\right), i=$ $1, \ldots, 4$, and using the fact that, by definition,

$$
\overline{\mathbf{Q}}_{1}=\frac{\omega_{1}}{z_{1}} \mathbf{D}^{-1 / 2}\left(\mathbf{C}-\omega_{1} \mathbf{I}\right)^{-1} \mathbf{D}^{-1 / 2}
$$

we obtain (82), where $\bar{\mu}(\omega)$ and $\mathcal{D}\left(\omega_{1}\right)$ are defined in (35) and (20) respectively.

\section{E. Analysis of the variance-related term $\tilde{\Theta}\left(z_{1}, z_{2}\right)$}

To finalize the proof of Theorem 2, it remains to show that $\tilde{\Theta}\left(z_{1}, z_{2}\right)$ can be asymptotically expressed as in (83). We will follow the same procedure as in the characterization of the mean-related term $\tilde{\mu}\left(z_{1}\right)$, by first decorrelating with respect to the characteristic function according to (91)-(92) and then investigating the asymptotic behavior of the resulting expectations. Recalling the form of $\tilde{\Theta}\left(z_{1}, z_{2}\right)$ in (80), we observe that we will need to characterize the expectation of traces of quantities containing three different stochastic matrices $\hat{\mathbf{Q}}(z)$. In particular, from the expression of $\tilde{\Theta}\left(z_{1}, z_{2}\right)$ we see that we need to characterize the asymptotic behavior of random quantities of the form

$$
\begin{aligned}
& \hat{\zeta}\left(\mathbf{A}_{1}, \mathbf{B}_{1}, \mathbf{B}_{2}\right)= \\
& \quad=\frac{1}{N} \operatorname{tr}\left[\hat{\mathbf{R}}\left(\mathbf{A}_{1} \hat{\mathbf{Q}}_{1} \odot \mathbf{B}_{1}\right) \mathbf{R}\left(\hat{\mathbf{Q}}_{2} \hat{\mathbf{R}} \hat{\mathbf{Q}}_{2} \odot \mathbf{B}_{2}\right) \phi\right]
\end{aligned}
$$

where $\mathbf{A}_{1}$ is a deterministic matrix with bounded spectral norm, and $\mathbf{B}_{1}, \mathbf{B}_{2}$ are two real-valued symmetric deterministic matrices with bounded entries. The following result provides a first step towards the characterization of the asymptotic mean and variance of $\hat{\zeta}\left(\mathbf{A}_{1}, \mathbf{B}_{1}, \mathbf{B}_{2}\right)$. We will differentiate four different situations, depending on the $\mathbf{B}_{1}, \mathbf{B}_{2}$ have bounded norm or are all-ones matrices.

Proposition 6: Let $\mathbf{A}_{1}$ have uniformly bounded spectral norm, and $\mathbf{B}_{1}, \mathbf{B}_{2}$ Hermitian positive semidefinite and with bounded entries. Then, $\operatorname{var}\left[\hat{\zeta}\left(\mathbf{A}_{1}, \mathbf{B}_{1}, \mathbf{B}_{2}\right)\right]=\mathcal{O}\left(N^{-2}\right)$.
Furthermore, if both $\mathbf{B}_{1}$ and $\mathbf{B}_{2}$ have bounded spectral norm, we have

$$
\begin{aligned}
& \mathbb{E}\left[\hat{\zeta}\left(\mathbf{A}_{1}, \mathbf{B}_{1}, \mathbf{B}_{2}\right)\right]=\frac{\left(z_{2} / \omega_{2}\right)^{2}}{1-\Gamma_{22}} \times \\
\times & \frac{1}{N} \mathbb{E} \operatorname{tr}\left[\mathbf{R}\left(\mathbf{A}_{1} \overline{\mathbf{Q}}_{1} \odot \mathbf{B}_{1}\right) \mathbf{R}\left(\overline{\mathbf{Q}}_{2} \mathbf{R} \overline{\mathbf{Q}}_{2} \odot \mathbf{B}_{2}\right)\right]+\mathcal{O}\left(N^{-1}\right)
\end{aligned}
$$

On the other hand, if $\mathbf{B}_{1}=\mathbf{1 1}^{T}$ is an all-ones matrix and $\mathbf{B}_{2}$ has bounded spectral norm,

$$
\begin{aligned}
& \mathbb{E}\left[\hat{\zeta}\left(\mathbf{A}_{1}, \mathbf{1 1}^{T}, \mathbf{B}_{2}\right)\right]= \\
& =\frac{\left(z_{2} / \omega_{2}\right)^{2}}{1-\Gamma_{22}} \frac{1}{N} \mathbb{E} \operatorname{tr}\left[\mathbf{R} \mathbf{A}_{1} \overline{\mathbf{Q}}_{1} \mathbf{R}\left(\overline{\mathbf{Q}}_{2} \mathbf{R} \overline{\mathbf{Q}}_{2} \odot \mathbf{B}_{2}\right)\right] \\
& \quad-\frac{\left(z_{1} / \omega_{1}\right)\left(z_{2} / \omega_{2}\right)^{2}}{1-\Gamma_{22}} \frac{1}{N} \operatorname{tr}\left[\mathbf{A}_{1} \overline{\mathbf{Q}}_{1} \mathbf{R}\right] \times \\
& \quad \times \frac{1}{N} \mathbb{E} \operatorname{tr}\left[\mathbf{R} \overline{\mathbf{Q}}_{1} \mathbf{R}\left(\overline{\mathbf{Q}}_{2} \mathbf{R} \overline{\mathbf{Q}}_{2} \odot \mathbf{B}_{2}\right)\right]+\mathcal{O}\left(N^{-1}\right)
\end{aligned}
$$

whereas if $\mathbf{B}_{2}=\mathbf{1 1}^{T}$ and $\mathbf{B}_{1}$ has bounded spectral norm,

$$
\begin{aligned}
\mathbb{E}\left[\hat{\zeta}\left(\mathbf{A}_{1}, \mathbf{B}_{1}, \mathbf{1 1}^{T}\right)\right]= \\
=\frac{\left(z_{2} / \omega_{2}\right)^{3}}{1-\Gamma_{22}} \frac{1}{N} \mathbb{E} \operatorname{tr}\left[\mathbf{R}\left(\mathbf{A}_{1} \overline{\mathbf{Q}}_{1} \odot \mathbf{B}_{1}\right) \mathbf{R}\left(\overline{\mathbf{Q}}_{2} \mathbf{R} \overline{\mathbf{Q}}_{2}\right)\right] \\
\quad+\frac{z_{2}}{\omega_{2}}\left(1-\frac{z_{2}}{\omega_{2}} \frac{1}{1-\Gamma_{22}}\right) \times \\
\quad \times \frac{1}{N} \mathbb{E} \operatorname{tr}\left[\mathbf{R} \overline{\mathbf{Q}}_{2} \mathbf{R}\left(\mathbf{A}_{1} \overline{\mathbf{Q}}_{1} \odot \mathbf{B}_{1}\right)\right]+\mathcal{O}\left(N^{-1}\right) .
\end{aligned}
$$

Finally, if $\mathbf{B}_{1}=\mathbf{B}_{2}=\mathbf{1 1}^{T}$, we have

$$
\begin{aligned}
& \mathbb{E}\left[\hat{\zeta}\left(\mathbf{A}_{1}, \mathbf{1 1}^{T}, \mathbf{1 1}^{T}\right)\right]= \\
& =\frac{\left(z_{2} / \omega_{2}\right)^{3}}{\left(1-\Gamma_{22}\right)\left(1-\Gamma_{12}\right)} \frac{1}{N} \operatorname{tr}\left[\mathbf{R}\left(\mathbf{A}_{1} \overline{\mathbf{Q}}_{1}\right) \mathbf{R}\left(\overline{\mathbf{Q}}_{2} \mathbf{R} \overline{\mathbf{Q}}_{2}\right)\right] \\
& \quad+\left(\frac{z_{2}}{\omega_{2}} \frac{1}{N} \mathbb{E} \operatorname{tr}\left[\mathbf{A}_{1} \overline{\mathbf{Q}}_{1} \mathbf{R} \overline{\mathbf{Q}}_{2} \mathbf{R}\right]-\frac{1}{N} \operatorname{tr}\left[\mathbf{A}_{1} \overline{\mathbf{Q}}_{1} \mathbf{R}\right]\right) \times \\
& \quad \times \frac{z_{1} / \omega_{1}}{1-\Gamma_{22}} \frac{\left(z_{2} / \omega_{2}\right)^{3}}{\left(1-\Gamma_{12}\right)^{2}} \frac{1}{N} \operatorname{tr}\left[\mathbf{R} \overline{\mathbf{Q}}_{1} \mathbf{R}\left(\overline{\mathbf{Q}}_{2} \mathbf{R} \overline{\mathbf{Q}}_{2}\right)\right] \\
& +\frac{z_{2} / \omega_{2}}{1-\Gamma_{12}}\left(1-\frac{z_{2} / \omega_{2}}{\left(1-\Gamma_{22}\right)\left(1-\Gamma_{12}\right)}\right) \frac{1}{N} \operatorname{tr}\left[\mathbf{R} \overline{\mathbf{Q}}_{2} \mathbf{R} \mathbf{A}_{1} \overline{\mathbf{Q}}_{1}\right] \\
& -\left(1-\frac{z_{2} / \omega_{2}}{\left(1-\Gamma_{22}\right)\left(1-\Gamma_{12}\right)}\right) \frac{\Gamma_{12}}{1-\Gamma_{12}} \frac{1}{N} \operatorname{tr}\left[\mathbf{A}_{1} \overline{\mathbf{Q}}_{1} \mathbf{R}\right] \\
& \quad+\mathcal{O}\left(N^{-1}\right) \quad(102)
\end{aligned}
$$

Proof: See Appendix D.

Using this proposition together with Propositions 3 and 4, we can readily establish that $\tilde{\Theta}\left(z_{1}, z_{2}\right)$ is as in (103) at the top of next page, where $\Delta=\left(1-\Gamma_{11}\right)^{-1}\left(1-\Gamma_{22}\right)^{-1}$ and

$$
\begin{aligned}
& \bar{\Upsilon}_{1}=\overline{\mathbf{Q}}_{1} \mathbf{D} \overline{\mathbf{Q}}_{1}-\operatorname{dg}\left(\overline{\mathbf{Q}}_{1} \mathbf{R} \overline{\mathbf{Q}}_{1}\right) \\
& \bar{\Upsilon}_{2}=\overline{\mathbf{Q}}_{2} \mathbf{D} \overline{\mathbf{Q}}_{2}-\operatorname{dg}\left(\overline{\mathbf{Q}}_{2} \mathbf{R} \overline{\mathbf{Q}}_{2}\right) .
\end{aligned}
$$

We can further simply this expression by assuming that $z_{1} \neq$ $z_{2}$. It can readily be checked that

$$
\left(z_{1}-z_{2}\right)=\left(\omega_{1}-\omega_{2}\right)\left(1-\Gamma_{12}\right)
$$




$$
\begin{aligned}
\tilde{\Theta}\left(z_{1}, z_{2}\right)=\frac{\Delta(1+\varsigma) \Gamma_{12}}{\left(1-\Gamma_{12}\right)}\left(\frac{z_{1} z_{2}}{\omega_{1} \omega_{2}}\right)^{2} \frac{1}{N} \operatorname{tr}\left[\mathbf{R}\left(\overline{\mathbf{Q}}_{1} \mathbf{D} \overline{\mathbf{Q}}_{1}\right) \mathbf{R}\left(\overline{\mathbf{Q}}_{2} \mathbf{D} \overline{\mathbf{Q}}_{2}\right)\right] \mathbb{E}[\Psi] \\
+\frac{\Delta(1+\varsigma)}{\left(1-\Gamma_{12}\right)^{2}}\left(\frac{z_{1} z_{2}}{\omega_{1} \omega_{2}}\right)^{3} \frac{1}{N} \mathbb{E} \operatorname{tr}\left[\mathbf{R}\left(\overline{\mathbf{Q}}_{1} \mathbf{D} \overline{\mathbf{Q}}_{1}\right) \mathbf{R} \overline{\mathbf{Q}}_{2}\right] \frac{1}{N} \operatorname{tr}\left[\mathbf{R} \overline{\mathbf{Q}}_{1} \mathbf{R}\left(\overline{\mathbf{Q}}_{2} \mathbf{D} \overline{\mathbf{Q}}_{2}\right)\right] \mathbb{E}[\Psi] \\
+\Delta\left(\frac{z_{1} z_{2}}{\omega_{1} \omega_{2}}\right)^{2} \frac{1+\varsigma}{N} \operatorname{tr}\left[\mathbf{R} \bar{\Upsilon}_{1} \mathbf{R} \bar{\Upsilon}_{2}\right] \mathbb{E}[\Psi]+\mathcal{O}\left(N^{-1}\right)
\end{aligned}
$$

and therefore we may assume that $\omega_{1} \neq \omega_{2}$ whenever $z_{1}$ and $z_{2}$ are located on the contour $\mathcal{C}$, because $\left|\Gamma_{12}\right|<1$ in that situation (cf. (76)). Furthermore, one can easily check that the following identities hold true

$$
\begin{gathered}
\frac{1}{N} \operatorname{tr}\left[\mathbf{R}\left(\overline{\mathbf{Q}}_{1} \mathbf{D} \overline{\mathbf{Q}}_{1}\right) \mathbf{R}\left(\overline{\mathbf{Q}}_{2} \mathbf{D} \overline{\mathbf{Q}}_{2}\right)\right]=\frac{\Gamma_{11}+\Gamma_{22}-2 \Gamma_{12}}{\left(z_{1} z_{2}\right)^{2}\left(\omega_{1}^{-1}-\omega_{2}^{-1}\right)^{2}} \\
\left(\frac{z_{1}}{\omega_{1}}\right)^{2} \frac{z_{2}}{\omega_{2}} \frac{1+\varsigma}{N} \mathbb{E} \operatorname{tr}\left[\mathbf{R}\left(\overline{\mathbf{Q}}_{1} \mathbf{D} \overline{\mathbf{Q}}_{1}\right) \mathbf{R} \overline{\mathbf{Q}}_{2}\right]=\frac{\Gamma_{11}-\Gamma_{12}}{\omega_{1}-\omega_{2}} .
\end{gathered}
$$

Using these identities in (103) and noting that $\Delta=$ $\mathcal{D}^{-1}\left(\omega_{1}\right) \mathcal{D}^{-1}\left(\omega_{2}\right)$ we obtain (83) with

$$
h\left(z_{1}, z_{2}\right)=\frac{-1}{\left(z_{1}-z_{2}\right)^{2}} .
$$

Observing that the integral in (85) is zero for this definition of $h\left(z_{1}, z_{2}\right)$, we conclude the proof of this theorem.

\section{Conclusions}

A central limit theorem for linear spectral statistics of the sample correlation matrix has been obtained under the assumption that both the sample size and the observation dimension increase to infinity at the same rate. The theorem holds for both real-valued and complex-valued Gaussian observations with a general covariance structure (not necessarily proportional to the identity) and can be applied to both the undersampled and oversampled regimes. The result has been particularized to the GLRT and FNT statistics, which are designed to determine the presence of correlation among multiple -non-necessarily identically distributed- Gaussian signals. It has been shown that under both null and alternative hypothesis, these statistics asymptotically fluctuate as Gaussian random variables, with a mean and covariance that can be expressed in compact analytical form. The analysis provided could be used in order to establish the optimum choice of function $f(\cdot)$ in the LSS in order to guarantee the best asymptotic performance in the limit as $M, N \rightarrow \infty$ at the same rate. However, this appears challenging from the mathematical perspective, given the complicated dependence of the asymptotic mean and variance on this function. Finally, it would be interesting to compare the asymptotic performance of correlation tests based on LSS of the sample correlation matrix with those based on LSS of the sample covariance matrix. Note that the results derived in this paper are in direct relationship with those provided for the sample covariance matrix in [24], so it seems feasible to compare different correlation detection tests based on these two different random matrix models. This is left for further research.

\section{ACKNOWLEDGMENT}

The authors would like to thank Walid Hachem and Romain Couillet for the useful technical discussions and feedback, which helped to improve the quality of the paper.

\section{APPENDIX A \\ PROOF OF (9)}

In this appendix, we prove that $\hat{\mathbf{D}}_{M}-\mathbf{D}_{M}$ converges to zero in spectral norm. Since the spectral norm of a diagonal matrix is the maximum absolute value of its diagonal entries, we only need to prove that

$$
\max _{1 \leq k \leq M}\left\{\left|\hat{d}_{k, M}-d_{k, M}\right|\right\} \rightarrow 0 \text { a.s. }
$$

where $\hat{d}_{k, N}$ and $d_{k, N}$ represent the $k$ th diagonal entry of $\hat{\mathbf{D}}_{M}$ and $\mathbf{D}_{M}$ respectively. To see the above, we observe that for any $\epsilon>0$ we can write

$$
\begin{aligned}
\mathbb{P}\left(\max _{1 \leq k \leq M}\left\{\left|\hat{d}_{k, M}-d_{k, M}\right|\right\}>\epsilon\right) \leq \\
\leq \sum_{k=1}^{M} \mathbb{P}\left(\left|\hat{d}_{k, M}-d_{k, M}\right|>\epsilon\right) .
\end{aligned}
$$

On the other hand, observe that we can express

$$
\hat{d}_{k, M}=\mathbf{e}_{k}^{H} \mathbf{R}^{1 / 2} \frac{\mathbf{X X}}{N} \mathbf{R}^{1 / 2} \mathbf{e}_{k}=d_{k, M} \frac{\chi^{2}((1+\varsigma) N)}{(1+\varsigma) N}
$$

where $\chi^{2}(n)$ is a chi-square distributed random variable with $n$ degrees of freedom. Consequently,

$$
\begin{aligned}
\mathbb{E}\left[\left|\hat{d}_{k, M}-d_{k, M}\right|^{5}\right] & =\left|d_{k, M}\right|^{5} \mathbb{E}\left[\left|\frac{\chi^{2}((1+\varsigma) N)}{(1+\varsigma) N}-1\right|^{5}\right] \\
& =\mathcal{O}\left(N^{-3}\right)
\end{aligned}
$$

By Markov's inequality,

$$
\mathbb{P}\left(\max _{1 \leq k \leq M}\left\{\left|\hat{d}_{k, M}-d_{k, M}\right|\right\}>\epsilon\right) \leq \mathcal{O}\left(N^{-2}\right)
$$

and the result follows from the Borel-Cantelli lemma.

\section{APPENDIX B \\ PROOF OF PROPOSITION 1}

In order to prove Proposition 1, we follow the procedure established in [36] for similar integrals. Observe that all the integrals of Proposition 1 can be generally expressed in the form

$$
\mathcal{J}_{h}=\frac{1}{2 \pi \mathrm{j}} \oint_{C_{\omega}^{+}} h(\omega) \mathcal{L}(\omega) d \omega
$$


where $\mathcal{L}(\omega)$ is as defined in (25) -ommiting the dependence on $M-$ and where $h(\omega)$ is a general complex function. By noticing that $\mu_{0}$ is a root of (26), we can subtract the left hand side of (26) evaluated at $\mu=\mu_{0}$ from the argument of $\mathcal{L}(\omega)$ and equivalently write

$\mathcal{L}(\omega)=\log \left[\left(\omega-\mu_{0}\right)\left(1-\frac{1}{N} \sum_{m=1}^{\bar{M}} \frac{K_{m} \gamma_{m}^{2}}{\left(\gamma_{m}-\omega\right)\left(\gamma_{m}-\mu_{0}\right)}\right)\right]$

The main advantage of this expression with respect to the one in (25) comes from the fact that $\omega-\mu_{0}$ is always ensured to have positive real part for all $\omega$ in $C_{\omega}^{+}$, regardless of whether we are in the undersampled or the oversampled regime. This will tremendously simplify some of the integration steps that follow.

Let us briefly summarize the approach in [36]. Observe that the integral in (104) can be obtained by evaluating at $x=1$ the function $\mathcal{J}_{h}(x):[0,1] \rightarrow \mathbb{R}$, defined as

$$
\mathcal{J}_{h}(x)=\frac{1}{2 \pi \mathrm{j}} \oint_{C_{\omega}^{+}} h(\omega) \mathcal{L}(\omega, x) d \omega
$$

where

$$
\mathcal{L}(\omega, x)=\log \left[\left(\omega-\mu_{0}\right)\left(1-\frac{x}{N} \sum_{m=1}^{\bar{M}} \widetilde{K}_{m} \frac{\gamma_{m}}{\gamma_{m}-\omega}\right)\right]_{0}
$$

and where $\widetilde{K}_{m}, m=1, \ldots, \bar{M}$, are the following positive weights:

$$
\widetilde{K}_{m}=K_{m} \frac{\gamma_{m}}{\gamma_{m}-\mu_{0}} .
$$

The main idea behind the approach in [36] comes from the observation that $\mathcal{J}_{h}(x)$ is a differentiable function of $x$, and that both $\mathcal{J}_{h}(0)$ and $d \mathcal{J}_{h}(x) / d x$ can be easily computed using conventional residue calculus. Therefore, one can find the value of $\mathcal{J}_{h}(1)$ by finding a primitive of $\mathcal{J}_{h}^{\prime}(x)$ and using $\mathcal{J}_{h}(0)$ to fix the undetermined constant. We will begin with the following lemma, which establishes the differentiability of $\mathcal{J}_{h}(x)$.

Lemma 4: Let $h(\omega)$ denote a complex function such that

$$
\sup _{\omega \in C_{\omega}^{+}}|h(\omega)|<\infty \text {. }
$$

The integral

$$
\mathcal{J}_{h}(x)=\frac{1}{2 \pi \mathrm{j}} \oint_{C_{\omega}^{+}} h(\omega) \mathcal{L}(\omega, x) d \omega
$$

is a differentiable function of $x$ with derivative

$$
\begin{aligned}
\mathcal{J}_{h}^{\prime}(x) & =\frac{1}{2 \pi \mathrm{j}} \oint_{C_{\omega}^{+}} h(\omega) \frac{\partial \mathcal{L}(\omega, x)}{\partial x} d \omega \\
& =\frac{-1}{2 \pi \mathrm{j}} \oint_{C_{\omega}^{+}} h(\omega)\left(1-\frac{x}{N} \sum_{m=1}^{\bar{M}} \widetilde{K}_{m} \frac{\gamma_{m}}{\gamma_{m}-\omega}\right)^{-1} \times \\
& \times \frac{1}{N} \sum_{m=1}^{\bar{M}} \widetilde{K}_{m} \frac{\gamma_{m}}{\gamma_{m}-\omega} d \omega
\end{aligned}
$$

where $\widetilde{K}_{m}$ is defined in (106).

Proof: Note that $\mathcal{J}_{h}^{\prime}(x)$ is well defined for some $x \in$ $[0,1]$, for example for $x=0$. On the other hand, the partial derivative $\frac{\partial \mathcal{L}(\omega, x)}{\partial x}$ exists for all $C_{\omega}^{+} \times[0,1]$ and is absolutely bounded by an integrable function for all $x$. To see this, we need to use the triangular inequality to express

$$
\begin{aligned}
\left|\frac{\partial \mathcal{L}(\omega, x)}{\partial x}\right| & \leq\left(1-\left|\frac{1}{N} \sum_{m=1}^{\bar{M}} \widetilde{K}_{m} \frac{\gamma_{m}}{\gamma_{m}-\omega}\right|\right)^{-1} \times \\
& \times\left|\frac{1}{N} \sum_{m=1}^{\bar{M}} \widetilde{K}_{m} \frac{\gamma_{m}}{\gamma_{m}-\omega}\right|
\end{aligned}
$$

which is clearly bounded for all $\omega \in C_{\omega}^{+}$. Indeed, by the Cauchy-Schwarz inequality we will have

$$
\begin{aligned}
\left|\frac{1}{N} \sum_{m=1}^{\bar{M}} \widetilde{K}_{m} \frac{\gamma_{m}}{\gamma_{m}-\omega}\right|^{2} \leq & \frac{1}{N} \sum_{m=1}^{\bar{M}} K_{m}\left(\frac{\gamma_{m}}{\gamma_{m}-\mu_{0}}\right)^{2} \times \\
& \times \frac{1}{N} \sum_{m=1}^{\bar{M}} K_{m}\left|\frac{\gamma_{m}}{\gamma_{m}-\omega}\right|^{2}<1
\end{aligned}
$$

where the last inequality follows from the fact that $\omega \in C_{\omega}^{+}$ and from the the fact that $\mu_{0}$ always lies outside $C_{\omega}^{+}$(cf. [35, eq. (49)]). A direct application of the Dominated Convergence Theorem concludes the proof of this lemma.

Next, we proceed to the application of the above lemma in order to obtain the different integrals in Proposition 1.

\section{A. Proof of (27)}

Observe that this integral can be expressed as $\mathcal{I}_{0}(1)$, where

$$
\mathcal{I}_{0}(x)=\frac{1}{2 \pi \mathrm{j}} \oint_{C_{\omega}^{+}} \mathcal{L}(\omega, x) d \omega .
$$

By Lemma 4 we know that this function is differentiable on $(0,1)$. Using the fact that $\operatorname{Re}\left(\omega-\mu_{0}\right)$ is always positive for all $\omega$ in $C_{\omega}^{+}$we obtain

$$
\mathcal{I}_{0}(0)=\frac{1}{2 \pi \mathrm{j}} \oint_{\mathcal{C}_{\omega}^{+}} \log \left(\omega-\mu_{0}\right) d \omega=0
$$

whereas

$$
\mathcal{I}_{0}^{\prime}(x)=-\sum_{k=1}^{\bar{M}} \mu_{k}^{\prime}(x)
$$

where $\mu_{k}^{\prime}(x)$ is the derivative of $\mu_{k}(x)$, and $\mu_{k}(x), k=$ $1, \ldots, \bar{M}$, are defined as the ordered solutions to

$$
1=\frac{x}{N} \sum_{m=1}^{\bar{M}} \widetilde{K}_{m} \frac{\gamma_{m}}{\gamma_{m}-\mu_{k}(x)} .
$$

Observe that there exist exactly $\bar{M}$ solutions, and that they are always located inside ${ }^{3} \mathcal{C}_{\omega}^{+}$. Note also each $\mu_{k}(x)$ is a simple root of a polynomial equation, and is therefore differentiable on $(0,1)$, so $\mu_{k}^{\prime}(x)$ is well defined.

\footnotetext{
${ }^{3}$ Indeed, one can readily check that $\mu_{k}(x) \in\left(\mu_{k}, \gamma_{k}\right)$ when $x \in(0,1)$ and since the segment $\left(\mu_{k}, \gamma_{k}\right)$ is always enclosed by $\mathcal{C}_{\omega}^{+}$, so will be $\mu_{k}(x)$.
} 
Taking the primitive of $\mathcal{I}_{0}^{\prime}(x)$ with respect to $x$, we see that

$$
\mathcal{I}_{0}(x)=-\sum_{k=1}^{\bar{M}} \mu_{k}(x)+C
$$

for some unknown constant $C$, which can be determined by forcing $\mathcal{I}_{0}(0)=0$ and noting that $\mu_{k}(x) \rightarrow \gamma_{k}$ when $x \rightarrow 0$. Hence,

$$
\mathcal{I}_{0}=\mathcal{I}_{0}(1)=-\sum_{k=1}^{\bar{M}} \mu_{k}+\sum_{k=1}^{\bar{M}} \gamma_{k}
$$

where $\mu_{k}=\mu_{k}(1), k=1, \ldots, \bar{M}$, correspond to the roots of (26). In order to obtain the expression of this integral in Proposition 1, we use the fact that $\mu_{0}$ is a solution to the equation in (26) and factorize this equation as

$$
\left(\mu-\mu_{0}\right)\left(1-\frac{1}{N} \sum_{m=1}^{\bar{M}} K_{m} \frac{\gamma_{m}^{2}}{\left(\gamma_{m}-\mu\right)\left(\gamma_{m}-\mu_{0}\right)}\right)=0
$$

By dropping the term $\left(\mu-\mu_{0}\right)$ we obtain an equation defining the $\bar{M}$ largest roots of the original equation in (26). This is a polynomial equation that has roots $\mu_{\ell}, \ell=1, \ldots, \bar{M}$, so that we may identify the following two polynomials

$$
\begin{aligned}
\prod_{\ell=1}^{\bar{M}}\left(\mu_{\ell}-\mu\right)= & \prod_{\ell=1}^{\bar{M}}\left(\gamma_{\ell}-\mu\right) \\
& -\frac{1}{N} \sum_{m=1}^{\bar{M}} \frac{K_{m} \gamma_{m}^{2}}{\left(\gamma_{m}-\mu_{0}\right)} \prod_{\substack{\ell=1 \\
\ell \neq m}}^{\bar{M}}\left(\gamma_{\ell}-\mu\right) .
\end{aligned}
$$

By isolating the coefficients of the terms in $\mu^{\bar{M}-1}$ on both sides of the above equation, we obtain the identity

$$
\sum_{k=1}^{\bar{M}} \mu_{k}=\sum_{k=1}^{\bar{M}} \gamma_{k}-\frac{1}{N} \sum_{m=1}^{\bar{M}} \frac{K_{m} \gamma_{m}^{2}}{\left(\gamma_{m}-\mu_{0}\right)}
$$

which directly leads to the expression of (27) in Proposition 1 .

\section{B. Proof of (28)-(29)}

We want to compute $\mathcal{I}_{1}(1)$, where $\mathcal{I}_{1}(x)$ is a function on the unit interval given by

$$
\mathcal{I}_{1}(x)=\frac{1}{2 \pi \mathrm{j}} \oint_{\mathcal{C}_{\omega}^{+}} \frac{\mathcal{L}(\omega, x)}{\omega-\zeta} d \omega
$$

where $\zeta$ is a certain complex number not belonging to the contour $\mathcal{C}_{\omega}^{+}$. We know from Lemma 4 that the above function is differentiable because of the boundedness of $h(\omega)=$ $(\omega-\zeta)^{-1}$ on the contour. Therefore, we can apply the strategy in [36] once again. We will differentiate between two cases $\zeta$ inside $\mathcal{C}_{\omega}^{+}$and $\zeta$ outside $\mathcal{C}_{\omega}^{+}$.
1) The value $\zeta$ lies outside $\mathcal{C}_{\omega}^{+}$: Assume first that $\zeta$ lies outside the contour $\mathcal{C}_{\omega}^{+}$. In this situation, we can write

$$
\mathcal{I}_{1}(0)=\frac{1}{2 \pi \mathrm{j}} \oint_{\mathcal{C}_{\omega}^{+}} \frac{1}{\omega-\zeta} \log \left(\omega-\mu_{0}\right) d \omega=0
$$

which follows from the fact that the integrand is holomorphic inside the contour, since $\mu_{0}$ is always outside $\mathcal{C}_{\omega}^{+}$. As for the derivative of $\mathcal{I}_{1}(x)$, classical residue computation leads to

$$
\mathcal{I}_{1}^{\prime}(x)=-\sum_{k=1}^{\bar{M}} \frac{1}{\mu_{k}(x)-\zeta} \mu_{k}^{\prime}(x)
$$

where $\mu_{k}(x), k=1, \ldots, \bar{M}$, are solutions to (107). In order to find the primitive of the above function, we need to differentiate between different regions for the location of $\zeta$. If $\zeta \in \mathbb{C} \backslash\left(\mathbb{R}^{+} \cup\{0\}\right)$, we can write the primitive of the above function as

$$
\mathcal{I}_{1}(x)=-\sum_{k=1}^{\bar{M}} \log \left(\mu_{k}(x)-\zeta\right)+C
$$

for some constant $C$, where $\log (\cdot)$ is the principal branch of the complex logarithm. Forcing $\mathcal{I}_{1}(0)=0$ we obtain the value of $C$, and using the identity in (108) evaluated at $\mu=\zeta$ we conclude that

$$
\mathcal{I}_{1}=\mathcal{I}_{1}(1)=-\log \left(1-\frac{1}{N} \sum_{m=1}^{\bar{M}} \frac{K_{m} \gamma_{m}^{2}}{\left(\gamma_{m}-\mu_{0}\right)\left(\gamma_{m}-\zeta\right)}\right)
$$

Assume now that $\zeta \in \mathbb{R}^{+} \cup\{0\}$. In that case, the primitive is given by (109), but taking the absolute value of the argument of the logarithm. Operating as above, we reach the conclusion that

$$
\mathcal{I}_{1}=\mathcal{I}_{1}(1)=-\log \left|1-\frac{1}{N} \sum_{m=1}^{\bar{M}} \frac{K_{m} \gamma_{m}^{2}}{\left(\gamma_{m}-\mu_{0}\right)\left(\gamma_{m}-\zeta\right)}\right|
$$

However, the modulus of the argument of the logarithm can be dropped, because (by Cauchy-Schwarz)

$$
\begin{aligned}
\left|\frac{1}{N} \sum_{m=1}^{\bar{M}} \frac{K_{m} \gamma_{m}^{2}}{\left(\gamma_{m}-\mu_{0}\right)\left(\gamma_{m}-\zeta\right)}\right|^{2} & \leq \frac{1}{N} \sum_{m=1}^{\bar{M}} \frac{K_{m} \gamma_{m}^{2}}{\left(\gamma_{m}-\mu_{0}\right)^{2}} \times \\
& \times \frac{1}{N} \sum_{m=1}^{\bar{M}} \frac{K_{m} \gamma_{m}^{2}}{\left(\gamma_{m}-\zeta\right)^{2}}<1
\end{aligned}
$$

where the last inequality stems from the fact that both $\mu_{0}$ and $\zeta$ lie outside $\mathcal{C}_{\omega}^{+}$. Therefore, the same formula is valid for all values of $\zeta$ outside the contour.

2) The value $\zeta$ lies inside $\mathcal{C}_{\omega}^{+}$: In this situation, we can trivially obtain $\mathcal{I}_{1}(0)$ using classical residue calculus, namely

$$
\mathcal{I}_{1}(0)=\frac{1}{2 \pi \mathrm{j}} \oint_{\mathcal{C}_{\omega}^{+}} \frac{1}{\omega-\zeta} \log \left(\omega-\mu_{0}\right) d \omega=\log \left(\zeta-\mu_{0}\right) \text {. }
$$

Regarding the derivative $\mathcal{I}_{1}^{\prime}(x)$, the integration technique will ultimately depend on the value of $\zeta$. We will differentiate between two different cases, namely (1) $\zeta=\gamma_{\ell}$ for some $\ell$; and (2) $\zeta \notin\left(\mu_{\ell}, \gamma_{\ell}\right], \ell=1, \ldots, \bar{M}$. We will show that that in these two cases, the integral can be expressed as in (28). 
a) Case $\zeta=\gamma_{\ell}$ for some $\ell=1, \ldots, \bar{M}$ : In this case, the derivative of the original function $\mathcal{I}_{1}^{\prime}(x)$ can easily be computed using the classical residue theorem, namely

$$
\mathcal{I}_{1}^{\prime}(x)=\frac{1}{x}-\sum_{k=1}^{\bar{M}} \frac{1}{\mu_{k}(x)-\gamma_{\ell}} \mu_{k}^{\prime}(x)
$$

where, again, $\mu_{k}(x), k=1, \ldots, \bar{M}$, are solutions to (107). We recall that that $\mu_{\ell}(x) \in\left(\mu_{\ell}, \gamma_{\ell}\right)$ when $x \in(0,1)$. Finding a primitive of the above equation and forcing (110) to determine the indeterminate constant we obtain

$$
\mathcal{I}_{1}(x)=\sum_{\substack{k=1 \\ k \neq \ell}}^{\bar{M}} \log \left|\frac{\gamma_{\ell}-\gamma_{k}}{\gamma_{\ell}-\mu_{k}(x)}\right|+\log \left|\frac{K_{\ell} \gamma_{\ell}^{2}}{N} \frac{x}{\gamma_{\ell}-\mu_{\ell}(x)}\right|
$$

where we have used the fact that

$$
\lim _{x \rightarrow 0} \frac{\gamma_{k}-\mu_{k}(x)}{x}=\frac{K_{k}}{N} \frac{\gamma_{k}^{2}}{\gamma_{k}-\mu_{0}} .
$$

Therefore, the value of $\mathcal{I}_{1}$ is obtained by allowing $x \rightarrow 1$ in the above expression, which leads to

$$
\mathcal{I}_{1}=\sum_{\substack{k=1 \\ k \neq \ell}}^{\bar{M}} \log \left|\frac{\gamma_{\ell}-\gamma_{k}}{\gamma_{\ell}-\mu_{k}}\right|+\log \left|\frac{K_{\ell} \gamma_{\ell}^{2}}{N} \frac{1}{\gamma_{\ell}-\mu_{\ell}}\right| .
$$

Using the identity in (108) evaluated at $\mu=\gamma_{\ell}$ we obtain the value in (28).

b) Case $\zeta$ does not belong to any interval $\left(\mu_{\ell}, \gamma_{\ell}\right], \ell=$ $1, \ldots, \bar{M}$ : In this case, the derivative of the original function $\mathcal{I}_{1}^{\prime}(x), x \in(0,1)$, can be computed by obtaining the residues at the simple poles $\left\{\mu_{k}(x), k=1, \ldots, \bar{M}\right\}$ and $\{\zeta\}$. These are all different, because $\mu_{k}(x) \in\left(\mu_{k}, \gamma_{k}\right)$, and $\zeta$ does not belong to any one of these intervals. The derivative $\mathcal{I}_{1}^{\prime}(x)$ can be expressed as

$\mathcal{I}_{1}^{\prime}(x)=-\frac{\frac{1}{N} \sum_{m=1}^{\bar{M}} \widetilde{K}_{m} \frac{\gamma_{m}}{\gamma_{m}-\zeta}}{1-\frac{x}{N} \sum_{m=1}^{\bar{M}} \widetilde{K}_{m} \frac{\gamma_{m}}{\gamma_{m}-\zeta}}-\sum_{k=1}^{\bar{M}} \frac{1}{\mu_{k}(x)-\zeta} \mu_{k}^{\prime}(x)$.

In order to find the primitive of this cost function, we must differentiate between the different locations of $\zeta$.

Assume first that $\zeta$ belongs to $\mathbb{C} \backslash\left(\mathbb{R}^{+} \cup\{0\}\right)$. In this situation, we may write

$$
\begin{aligned}
\mathcal{I}_{1}(x) & =\log \left(1-\frac{x}{N} \sum_{m=1}^{\bar{M}} \widetilde{K}_{m} \frac{\gamma_{m}}{\gamma_{m}-\zeta}\right) \\
& -\sum_{k=1}^{\bar{M}} \log \left(\mu_{k}(x)-\zeta\right)+C
\end{aligned}
$$

for some constant $C$, where $\log (\cdot)$ is the principal branch of the complex logarithm. Indeed, observe that the argument of the second logarithm never lies on the negative real axis, because $\mu_{k}(x) \in\left(\mu_{k}, \gamma_{k}\right)$ and $\mu_{1} \geq 0$. As for the first term, we observe that the logarithm argument lies on the negative real axis if and only if $\zeta$ is real-valued and

$$
\frac{1}{N} \sum_{m=1}^{\bar{M}} \widetilde{K}_{m} \frac{\gamma_{m}}{\gamma_{m}-\zeta}>\frac{1}{x}
$$

implying that $\zeta \in\left(\mu_{\ell}, \gamma_{\ell}\right)$ for some $\ell$, which is in contradiction with our initial assumption. Hence, imposing (110), we see that for $\zeta \in \mathbb{C} \backslash\left(\mathbb{R}^{+} \cup\{0\}\right)$ we may write

$$
\begin{aligned}
\mathcal{I}_{1}(x)= & \log \left(1-\frac{x}{N} \sum_{m=1}^{\bar{M}} \widetilde{K}_{m} \frac{\gamma_{m}}{\gamma_{m}-\zeta}\right) \\
& +\log \left(\zeta-\mu_{0}\right)+\sum_{k=1}^{\bar{M}} \log \left(\frac{\gamma_{k}-\zeta}{\mu_{k}(x)-\zeta}\right) .
\end{aligned}
$$

Forcing $x \rightarrow 1$ in the above equation we see that

$$
\mathcal{I}_{1}(1)=\log \left(\zeta-\mu_{0}\right)
$$

where we have used the fact that $\zeta \in \mathbb{C} \backslash\left(\mathbb{R}^{+} \cup\{0\}\right)$ by assumption, together with the identity in (108) evaluated at $\mu=\zeta$.

Next, consider the case where $\zeta \in \mathbb{R}^{+} \cup\{0\}$. In this situation, the primitive of (112) takes the form in (113) but taking absolute value in the argument of the last logarithm. The value of the original integral is obtained by allowing $x \rightarrow 1$ in the above expression. Assume first that $\zeta=\mu_{\ell}$ for some $\ell \geq 1$. In this case, we can see that

$$
\mathcal{I}_{1}=\log \left(\mu_{\ell}-\mu_{0}\right)
$$

where we have used the fact that

$$
\lim _{x \rightarrow 1} \frac{\mu_{\ell}(x)-\mu_{\ell}}{1-x}=\left(\frac{1}{N} \sum_{m=1}^{\bar{M}} \widetilde{K}_{m} \frac{\gamma_{m}}{\left(\gamma_{m}-\mu_{\ell}\right)^{2}}\right)^{-1}
$$

together with the identity obtained by taking derivatives on both sides of (108) evaluating the result at $\mu=\mu_{\ell}$, namely

$$
\begin{aligned}
\prod_{r=1, r \neq \ell}^{\bar{M}}\left(\mu_{r}-\mu_{\ell}\right) & =\left(\frac{1}{N} \sum_{m=1}^{\bar{M}} \widetilde{K}_{m} \frac{\gamma_{m}}{\left(\gamma_{m}-\mu_{\ell}\right)^{2}}\right) \times \\
& \times \prod_{r=1}^{\bar{M}}\left(\gamma_{r}-\mu_{\ell}\right) .
\end{aligned}
$$

Finally, assume that $\zeta \notin\left\{\mu_{k}, k=1, \ldots, \bar{M}\right\}$. Following the same procedure, one can show that the formula in (28) is also valid in this case.

\section{Proof of (30) and (31)}

We will follow the same procedure as in the above derivations. The proof of (30) does not present any further complication and is therefore omitted. Regarding the proof of (31), we consider once again the function

$$
\mathcal{I}_{3}(x)=\frac{1}{2 \pi \mathrm{j}} \oint_{\mathcal{C}_{\omega}^{+}} \frac{\mathcal{L}(\omega, x)}{\left(\omega-\gamma_{q}\right)^{3}} d \omega
$$

where $\mathcal{L}(\omega, x)$ is defined in (105). Applying Lemma 4 with $h(\omega)=\left(\omega-\gamma_{q}\right)^{-3}$ we see that this function is differentiable on the unit interval. We can easily compute $\mathcal{I}_{3}(0)$ with conventional residue calculus, namely

$$
\mathcal{I}_{3}(0)=\frac{1}{2 \pi \mathrm{j}} \oint_{\mathcal{C}_{\omega}^{+}} \frac{\log \left(\omega-\mu_{0}\right)}{\left(\omega-\gamma_{q}\right)^{3}} d \omega=\frac{-1}{2\left(\gamma_{q}-\mu_{0}\right)^{2}} .
$$


As for the derivative of $\mathcal{I}_{3}(x)$, one trivially finds that

$$
\begin{aligned}
\mathcal{I}_{3}^{\prime}(x) & =\frac{N^{2}}{x^{3}}\left(\frac{\gamma_{q}-\mu_{0}}{K_{q} \gamma_{q}^{2}}\right)^{2}\left(1-\frac{x}{N} \sum_{\substack{m=1 \\
m \neq q}}^{\bar{M}} \widetilde{K}_{m} \frac{\gamma_{m}}{\gamma_{m}-\gamma_{q}}\right) \\
& -\sum_{k=1}^{\bar{M}} \frac{\mu_{k}^{\prime}(x)}{\left(\mu_{k}(x)-\gamma_{q}\right)^{3}}
\end{aligned}
$$

where $\mu_{k}(x), k=1, \ldots, \bar{M}$, are the solutions to (107). One can easily find a primitive of this function as

$$
\begin{aligned}
\mathcal{I}_{3}(x) & =-\frac{1}{2 x^{2}}\left(\frac{N\left(\gamma_{q}-\mu_{0}\right)}{K_{q} \gamma_{q}^{2}}\right)^{2}\left(1-2 x \frac{1}{N} \sum_{\substack{m=1 \\
m \neq q}}^{\bar{M}} \widetilde{K}_{m} \frac{\gamma_{m}}{\gamma_{m}-\gamma_{q}}\right) \\
& +\frac{1}{2} \sum_{k=1}^{\bar{M}} \frac{1}{\left(\mu_{k}(x)-\gamma_{q}\right)^{2}}+C
\end{aligned}
$$

for some constant $C$ that can be determined by forcing (114), namely

$$
\begin{aligned}
C & =-\frac{1}{2}\left(\frac{N\left(\gamma_{q}-\mu_{0}\right)}{K_{q} \gamma_{q}^{2}} \frac{1}{N} \sum_{\substack{m=1 \\
m \neq q}}^{\bar{M}} \widetilde{K}_{m} \frac{\gamma_{m}}{\gamma_{m}-\gamma_{q}}\right)^{2} \\
& -\frac{N\left(\gamma_{q}-\mu_{0}\right)}{K_{q} \gamma_{q}^{2}} \frac{1}{N} \sum_{\substack{m=1 \\
m \neq q}}^{\bar{M}} \widetilde{K}_{m} \frac{\gamma_{m}}{\left(\gamma_{m}-\gamma_{q}\right)^{2}} \\
& -\frac{1}{2} \sum_{\substack{k=1 \\
k \neq q}}^{\bar{M}} \frac{1}{\left(\gamma_{k}-\gamma_{q}\right)^{2}}-\frac{1}{2} \frac{1}{\left(\gamma_{q}-\mu_{0}\right)^{2}}
\end{aligned}
$$

where we have used (111) together with

$$
\begin{gathered}
\lim _{x \rightarrow 0}\left(\frac{1}{x}-\frac{1}{N} \frac{\widetilde{K_{k}} \gamma_{k}}{\gamma_{k}-\mu_{k}(x)}\right)=\frac{1}{N} \sum_{\substack{m=1 \\
m \neq k}}^{\bar{M}} \widetilde{K}_{m} \frac{\gamma_{m}}{\gamma_{m}-\gamma_{k}} \\
\lim _{x \rightarrow 0} \frac{1}{x}\left(\frac{1}{x}-\frac{1}{N} \frac{\widetilde{K_{k}} \gamma_{k}}{\gamma_{k}-\mu_{k}(x)}-\frac{1}{N} \sum_{\substack{m=1 \\
m \neq k}}^{\bar{M}} \widetilde{K}_{m} \frac{\gamma_{m}}{\gamma_{m}-\gamma_{k}}\right) \\
=-\frac{\widetilde{K_{k}} \gamma_{k}}{N^{2}} \sum_{\substack{m=1 \\
m \neq k}}^{\bar{M}} \widetilde{K}_{m} \frac{\gamma_{m}}{\left(\gamma_{m}-\gamma_{k}\right)^{2}} .
\end{gathered}
$$

Inserting the expression of $C$ into $I_{3}(x)$ and taking the limits as $x \rightarrow 1$ we obtain the final expression for $\mathcal{I}_{3}$. The formula in (31) is obtained by applying the following lemma.

Lemma 5: The identities and

$$
\begin{gathered}
\sum_{\substack{k=1 \\
k \neq q}}^{\bar{M}}\left(\frac{1}{\left(\gamma_{k}-\gamma_{q}\right)^{2}}-\frac{1}{\left(\mu_{k}-\gamma_{q}\right)^{2}}\right) \\
+\left[\sum_{\substack{k=1 \\
k \neq q}}^{\bar{M}}\left(\frac{1}{\gamma_{k}-\gamma_{q}}-\frac{1}{\mu_{k}-\gamma_{q}}\right)\right]^{2}= \\
=2 \frac{1}{\left(\gamma_{q}-\mu_{q}\right)} \sum_{\substack{k=1 \\
k \neq q}}^{\bar{M}}\left(\frac{1}{\gamma_{q}-\gamma_{k}}-\frac{1}{\gamma_{q}-\mu_{k}}\right) \\
-2 \frac{1}{\widetilde{K_{q}} \gamma_{q}} \sum_{\substack{k=1 \\
k \neq q}}^{\bar{M}} \widetilde{K_{k}} \frac{\gamma_{k}}{\left(\gamma_{k}-\gamma_{q}\right)^{2}}
\end{gathered}
$$

are valid for $q=1, \ldots, \bar{M}$.

Proof: The identity in (115) can be proven by taking first order derivatives of the polynomial identity in (108) and evaluating the result at $\mu=\gamma_{q}$, see further [35, Appendix IV] . The identity in (116) is obtained by taking second order derivatives at either side of (108), evaluating the result at $\mu=$ $\gamma_{q}$ and using (115) together with the identity

$$
\prod_{\ell=1}^{\bar{M}}\left(\mu_{\ell}-\gamma_{q}\right)=-\frac{\widetilde{K_{q}} \gamma_{q}}{N} \prod_{\ell=1, \ell \neq q}^{\bar{M}}\left(\gamma_{\ell}-\gamma_{q}\right)
$$

which is obtained by forcing $\mu=\gamma_{q}$ in (108).

\section{APPENDIX C}

\section{DERIVATION OF THE ASYMPTOTIC MEAN AND VARIANCES} OF THE GLRT AND FNT STATISTICS

In this appendix, we derive the asymptotic mean and variance for the two test statistics considered in this paper, namely the GLRT and the FNT statistics. The main tools are given in closed form expressions for the integrals in Lemma 3 and Proposition 1.

\section{A. Asymptotic mean and variance of the FNT statistic}

Regarding the asymptotic mean, we can divide the integral in (34) into two different parts, namely $\boldsymbol{\mu}_{M}=(1+\varsigma) \boldsymbol{\mu}_{M}^{(1)}+$ $\varsigma \boldsymbol{\mu}_{M}^{(2)}$, where

$$
\begin{aligned}
& \boldsymbol{\mu}_{M}^{(1)}= \frac{1}{2 \pi \mathrm{j}} \oint_{\mathcal{C}_{\omega}} \frac{1}{N} \operatorname{tr}\left[\left(\mathbf{C}_{M} \boldsymbol{\Theta}_{M}(\omega)-2 \mathbf{C}_{M}^{2} \boldsymbol{\Theta}_{M}^{2}(\omega)\right)\right. \\
&\left.\odot \mathbf{C}_{M} \boldsymbol{\Theta}_{M}(\omega)\right] \mathcal{Q}_{M}(\omega) d \omega \\
&+\frac{1}{2 \pi \mathrm{j}} \oint_{\mathcal{C}_{\omega}^{-}} \omega \frac{1}{N} \operatorname{tr}\left[\left(\mathbf{C}_{M} \boldsymbol{\Theta}_{M}^{2}(\omega) \odot \mathbf{C}_{M}\right) \times\right. \\
&\left.\times\left(\boldsymbol{\Theta}_{M}(\omega) \odot \mathbf{C}_{M}\right)\right] \mathcal{Q}_{M}(\omega) d \omega
\end{aligned}
$$

$$
\sum_{\substack{k=1 \\ k \neq q}}^{\bar{M}} \widetilde{K_{q}}\left(\frac{\gamma_{k}}{\gamma_{q}-\gamma_{k}}-\frac{\mu_{k}}{\gamma_{q}-\mu_{k}}\right)=\sum_{\substack{k=1 \\ k \neq q}}^{\bar{M}} \widetilde{K_{k}}\left(\frac{\gamma_{q}}{\gamma_{k}-\gamma_{q}}-\frac{\mu_{q}}{\gamma_{k}-\mu_{q}}\right){ }_{(115)}^{\text {and }} \quad \boldsymbol{\mu}_{M}^{(2)}=\frac{1}{2 \pi \mathrm{j}} \oint_{\mathcal{C}_{\omega}^{-}} \frac{\frac{1}{N} \operatorname{tr}\left[\mathbf{C}_{M}^{2} \boldsymbol{\Theta}_{M}^{3}(\omega)\right]}{1-\frac{1}{N} \operatorname{tr}\left[\mathbf{C}_{M}^{2} \boldsymbol{\Theta}_{M}^{2}(\omega)\right]} \mathcal{Q}_{M}(\omega) d \omega
$$


where $\mathcal{Q}_{M}(\omega)$ is defined in (22). To solve the first integral, we can use the eigen-decomposition of $\mathbf{C}_{M}$ together the partial fraction expansion of the resulting polynomial quotients and the integral results in Lemma 3, so that

$$
\boldsymbol{\mu}_{M}^{(1)}=\frac{1}{N} \operatorname{tr}\left[\left(\mathbf{C}_{M} \odot \mathbf{C}_{M}\right)^{2}\right]-2 \frac{1}{N} \operatorname{tr}\left[\mathbf{C}_{M}^{2}\right]
$$

where we have used the fact that $\left(\mathbf{I}_{M} \odot \mathbf{C}_{M}\right)=\mathbf{I}_{M}$.

In order to obtain a closed form expression for $\boldsymbol{\mu}_{M}^{(2)}$, we notice that the integrand is holomorphic except for the eigenvalues $\gamma_{m}, m=1, \ldots, \bar{M}$, and the $2 \bar{M}$ solutions to the equation

$$
1=\frac{1}{N} \operatorname{tr}\left[\mathbf{C}_{M}^{2} \boldsymbol{\Theta}_{M}^{2}(\omega)\right]
$$

which will be denoted as $\vartheta_{m}, m=1, \ldots, 2 \bar{M}$ (we allow for possible repetitions in case of multiplicities of order 2). It can be trivially seen that all these values are inside the contour $\mathcal{C}_{\omega}$. Indeed, the region outside $\mathcal{C}_{\omega}$ belongs to the set of points in the complex plane such that [35, eq. (49)]

$$
\omega \in \mathbb{C}: \frac{1}{N} \sum_{r=1}^{\bar{M}} K_{r} \frac{\gamma_{r}^{2}}{\left|\gamma_{r}-\omega\right|^{2}}<1
$$

Therefore, it suffices to prove that the points $\vartheta_{m}, m=$ $1, \ldots, 2 \bar{M}$ are such that

$$
\frac{1}{N} \sum_{r=1}^{\bar{M}} K_{r} \frac{\gamma_{r}^{2}}{\left|\gamma_{r}-\vartheta_{m}\right|^{2}}>1
$$

This is a consequence of the Cauchy-Schwarz inequality, because

$$
1=\frac{1}{N} \sum_{r=1}^{\bar{M}} K_{r}\left(\frac{\gamma_{r}}{\gamma_{r}-\vartheta_{m}}\right)^{2} \leq \frac{1}{N} \sum_{r=1}^{\bar{M}} K_{r} \frac{\gamma_{r}^{2}}{\left|\gamma_{r}-\vartheta_{m}\right|^{2}}
$$

and the fact that equality only holds for $z \in \mathcal{T}$, defined in Lemma 2, whereas $z \in \mathcal{C}$ by assumption (see also Appendix F). Therefore, the integral $\boldsymbol{\mu}_{M}^{(2)}$ can be evaluated by computing the residues at all these points, leading to

$$
\begin{gathered}
\boldsymbol{\mu}_{M}^{(2)} \sum_{m=1}^{\bar{M}}\left(\gamma_{m}\left(1-\frac{1}{N} \sum_{\substack{r=1 \\
r \neq m}}^{\bar{M}} K_{r} \frac{\gamma_{r}}{\gamma_{r}-\gamma_{m}}\right)+\frac{1}{N} K_{m} \gamma_{m}\right)^{2} \\
-3 \sum_{m=1}^{\bar{M}} \frac{1}{N} K_{m} \gamma_{m}^{2}\left(1-\frac{1}{N} \sum_{\substack{r=1 \\
r \neq m}}^{\bar{M}} K_{r} \frac{\gamma_{r}^{2}}{\left(\gamma_{r}-\gamma_{m}\right)^{2}}\right) \\
+\frac{1}{2} \sum_{m=1}^{2 \bar{M}} \vartheta_{m}^{2}\left(1-\frac{1}{N} \sum_{r=1}^{\bar{M}} K_{r} \frac{\gamma_{r}}{\gamma_{r}-\vartheta_{m}}\right)^{2}
\end{gathered}
$$

The expression of this integral can be further simplified by applying the following lemma.

Lemma 6: Let $\vartheta_{m}, m=1, \ldots, 2 \bar{M}$ denote all the roots of (119), with possible repetitions in case of higher order multiplicities. Then,

$$
\begin{aligned}
& \frac{1}{2} \sum_{m=1}^{2 \bar{M}} \vartheta_{m}=\sum_{m=1}^{\bar{M}} \gamma_{m} \\
& \frac{1}{2} \sum_{m=1}^{2 \bar{M}} \vartheta_{m}^{2}=\sum_{m=1}^{\bar{M}} \gamma_{m}^{2}+\frac{1}{N} \sum_{r=1}^{\bar{M}} K_{r} \gamma_{r}^{2} .
\end{aligned}
$$

Furthermore, for any $\ell=1, \ldots, \bar{M}$ we have

$$
\begin{aligned}
\frac{1}{2} \sum_{m=1}^{2 \bar{M}} \frac{1}{\vartheta_{m}-\gamma_{\ell}} & =\sum_{\substack{m=1 \\
m \neq \ell}}^{\bar{M}} \frac{1}{\gamma_{m}-\gamma_{\ell}} \\
\frac{1}{2} \sum_{m=1}^{2 \bar{M}} \frac{1}{\left(\vartheta_{m}-\gamma_{\ell}\right)^{2}} & =\sum_{\substack{m=1 \\
m \neq \ell}}^{\bar{M}} \frac{1}{\left(\gamma_{m}-\gamma_{\ell}\right)^{2}} \\
& +\frac{N}{K_{\ell} \gamma_{\ell}^{2}}\left(1-\frac{1}{N} \sum_{\substack{r=1 \\
r \neq \ell}}^{\bar{M}} K_{r} \frac{\gamma_{r}^{2}}{\left(\gamma_{r}-\gamma_{\ell}\right)^{2}}\right) .
\end{aligned}
$$

Proof: Consider the following polynomial identity, obtained by expressing (119) in polynomial form

$$
\begin{aligned}
\prod_{m=1}^{2 \bar{M}}\left(\vartheta_{m}-\vartheta\right) & =\prod_{m=1}^{\bar{M}}\left(\gamma_{m}-\vartheta\right)^{2} \\
& -\frac{1}{N} \sum_{r=1}^{\bar{M}} K_{r} \gamma_{r}^{2} \prod_{\substack{m \neq 1 \\
m \neq r}}^{\bar{M}}\left(\gamma_{m}-\vartheta\right)^{2}
\end{aligned}
$$

The identity in (121) is obtained by identifying the coefficients of the terms of order $2 \bar{M}-1$ on both sides of the above equation. On the other hand, the identification of the term of order $2 \bar{M}-2$ leads to (122). To obtain (123), simply take first order derivatives on both sides of (125) and evaluate the resulting polynomial identity at $\vartheta=\gamma_{\ell}$. Finally, taking second order derivatives on both sides of (125) and evaluating the result at $\vartheta=\gamma_{\ell}$ we obtain -after some algebra- (124).

Using the identities in Lemma 6, we are able to write

$$
\begin{aligned}
\frac{1}{2} \sum_{m=1}^{2 \bar{M}} \vartheta_{m}^{2} & \left(1-\frac{1}{N} \sum_{k=1}^{\bar{M}} K_{k} \frac{\gamma_{k}}{\gamma_{k}-\vartheta_{m}}\right)^{2}= \\
& =3 \frac{1}{N} \sum_{r=1}^{\bar{M}} K_{r} \gamma_{r}^{2}+\left(\frac{1}{N} \sum_{r=1}^{\bar{M}} K_{r} \gamma_{r}\right)^{2} \\
& +\sum_{m=1}^{\bar{M}} \gamma_{m}^{2}\left(1-\frac{1}{N} \sum_{\substack{k=1 \\
k \neq m}}^{\bar{M}} K_{k} \frac{\gamma_{k}}{\gamma_{k}-\gamma_{m}}\right)^{2} \\
& +3 \frac{1}{N} \sum_{k=1}^{\bar{M}} K_{k} \gamma_{k}^{2}\left(1-\frac{1}{N} \sum_{\substack{r=1 \\
r \neq k}}^{\bar{M}} K_{r} \frac{\gamma_{r}^{2}}{\left(\gamma_{r}-\gamma_{k}\right)^{2}}\right)
\end{aligned}
$$

Inserting this into (120) and using the identity (obtained by exploiting the symmetry with respect to the sum indexes)

$$
2 \sum_{m=1}^{\bar{M}} \sum_{\substack{r=1 \\ r \neq m}}^{\bar{M}} K_{r} K_{m} \frac{\gamma_{m}^{2} \gamma_{r}}{\gamma_{m}-\gamma_{r}}=\sum_{m=1}^{\bar{M}} \sum_{\substack{r=1 \\ r \neq m}}^{\bar{M}} K_{r} K_{m} \gamma_{m} \gamma_{r}
$$


we finally obtain

$$
\boldsymbol{\mu}_{M}^{(2)}=\frac{1}{N} \sum_{r=1}^{\bar{M}} K_{r} \gamma_{r}^{2}
$$

and therefore $\boldsymbol{\mu}_{M}$ as given in (38).

Regarding the asymptotic variance, we may re-write (36 ) as

$$
\Phi_{M}=\frac{-1}{4 \pi^{2}} \oint_{\mathcal{C}_{\omega_{1}}^{+}} \oint_{\mathcal{C}_{\omega_{2}}^{+}} \mathcal{Q}_{M}\left(\omega_{1}\right) \mathcal{Q}_{M}\left(\omega_{2}\right) \bar{\sigma}_{M}^{2}\left(\omega_{1}, \omega_{2}\right) d \omega_{1} d \omega_{2}
$$

where $\bar{\sigma}_{M}^{2}\left(\omega_{1}, \omega_{2}\right)$ is defined in (37) and where we have slightly deformed the contours so that $\mathcal{C}_{\omega_{1}}^{-}$is enclosed by $\mathcal{C}_{\omega_{2}}^{-}$. We can first compute the integral with respect to $\omega_{1}$, which will be denoted as

$$
\mathcal{I}_{M}\left(\omega_{2}\right)=\frac{1}{2 \pi \mathrm{j}} \oint_{\mathcal{C}_{\omega_{2}}^{+}} \mathcal{Q}_{M}\left(\omega_{1}\right) \bar{\sigma}_{M}^{2}\left(\omega_{1}, \omega_{2}\right) d \omega_{1}
$$

Using the identities in Lemma 3 and the eigendecomposition of $\mathbf{C}_{M}$ one can easily establish that

$$
\begin{aligned}
& \frac{1}{2 \pi \mathrm{j}} \oint_{\mathcal{C}_{\omega_{2}}^{+}} \mathcal{Q}_{M}\left(\omega_{1}\right) \frac{\bar{\sigma}_{M}^{2}\left(\omega_{1}, \omega_{2}\right)}{1+\varsigma} d \omega_{1} \\
& =2 \frac{1}{N} \sum_{m=1}^{\bar{M}} K_{m} \frac{\gamma_{m}^{3}}{\left(\gamma_{m}-\omega_{2}\right)^{2}}-2 \frac{1}{N^{2}} \sum_{m=1}^{\bar{M}} K_{m}^{2} \frac{\gamma_{m}^{3} \omega_{2}}{\left(\gamma_{m}-\omega_{2}\right)^{3}} \\
& +2 \frac{1}{N^{2}} \sum_{m=1}^{\bar{M}} \sum_{\substack{k=1 \\
k \neq m}}^{\bar{M}} K_{m} K_{k} \frac{\gamma_{m}^{3} \gamma_{k}}{\gamma_{m}-\gamma_{k}} \frac{1}{\left(\gamma_{m}-\omega_{2}\right)^{2}} \\
& -2 \frac{1}{N} \operatorname{tr}\left[\mathbf{C}_{M}^{3} \boldsymbol{\Theta}_{M}^{2}\left(\omega_{2}\right)\right]-2 \frac{c_{N}}{N} \operatorname{tr}\left[\mathbf{C}_{M}^{2} \boldsymbol{\Theta}_{M}^{2}\left(\omega_{2}\right)\right] \\
& +2 \frac{1}{N} \operatorname{tr}\left[\mathbf{C}_{M}\left[\mathbf{C}_{M}-\operatorname{dg}\left[\mathbf{C}_{M}^{2}\right]\right] \mathbf{C}_{M} \boldsymbol{\Delta}_{M}\left(\omega_{2}\right)\right]
\end{aligned}
$$

So, finally integrating with respect to $\omega_{2}$ (again, with the help of Lemma 3) and using the following two identities (obtained by exploiting the symmetry with respect to the sum indexes)

$$
\begin{aligned}
\frac{1}{N^{2}} \sum_{m=1}^{\bar{M}} \sum_{\substack{k=1 \\
k \neq m}}^{\bar{M}} K_{m} K_{k} \frac{\gamma_{m}^{3} \gamma_{k}}{\gamma_{m}-\gamma_{k}}= \\
\quad=\frac{c_{N}}{N} \sum_{m=1}^{\bar{M}} K_{m} \gamma_{m}^{2}-\frac{1}{N^{2}} \sum_{m=1}^{\bar{M}} K_{m}^{2} \gamma_{m}^{3}
\end{aligned}
$$

and

$$
\begin{aligned}
2 \frac{1}{N^{2}} \sum_{m=1}^{\bar{M}} \sum_{\substack{k=1 \\
k \neq m}}^{\bar{M}} K_{m} K_{k} \frac{\gamma_{m}^{4} \gamma_{k}}{\gamma_{m}-\gamma_{k}}=2 \frac{c_{N}}{N} \sum_{m=1}^{\bar{M}} K_{m} \gamma_{m}^{3} \\
-3 \frac{1}{N^{2}} \sum_{m=1}^{\bar{M}} K_{m}^{2} \gamma_{m}^{4}+\left(\frac{1}{N} \sum_{m=1}^{\bar{M}} K_{m} \gamma_{m}^{2}\right)^{2}
\end{aligned}
$$

we obtain (39).
1) Boundedness of the mean and variance: Using Lemma 1 we see that, under $(\mathbf{A s 2})-(\mathbf{A s 3})$, the mean $\boldsymbol{\mu}_{M}$ is absolutely bounded for all $M$, i.e.

$$
\begin{aligned}
\left|\boldsymbol{\mu}_{M}\right| \leq & (1+\varsigma) c_{M}\left\|\left(\mathbf{C}_{M} \odot \mathbf{C}_{M}\right)\right\|^{2} \\
& +(2+\varsigma) c_{M}\left\|\mathbf{C}_{M}\right\|^{2} \leq(3+2 \varsigma) c_{M} \theta^{2}<+\infty
\end{aligned}
$$

where the last inequality follows from the fact that the diagonal entries of $\mathbf{C}_{M}$ are all one and the fact that $\left\|\mathbf{C}_{M}\right\|^{2} \leq \theta^{2}$, with $\theta$ being defined in (12). Using the same reasoning, one can show that

$$
\left|\boldsymbol{\Phi}_{M}\right| \geq 2(1+\varsigma)\left(\frac{1}{N} \operatorname{tr}\left[\mathbf{C}_{M}^{2}\right]\right)^{2} \geq 2(1+\varsigma) \frac{c_{M}^{2}}{\theta^{4}}>0
$$

and also

$$
\begin{aligned}
& \left|\mathbf{\Phi}_{M}\right| \leq 2(1+\varsigma) c_{M}^{2}\left\|\mathbf{C}_{M}\right\|^{4} \\
& \quad+4(1+\varsigma) c_{M}\left\|\mathbf{C}_{M}\right\|^{2}\left(\left\|\mathbf{C}_{M}\right\|+\left\|\mathbf{C}_{M}^{2}\right\|\right)^{2}<+\infty
\end{aligned}
$$

\section{B. Asymptotic mean and variance of the GLRT statistic}

As in the past subsection, we divide the mean into two integrals, i.e. $\boldsymbol{\mu}_{M}=(1+\varsigma) \boldsymbol{\mu}_{M}^{(1)}+\varsigma \boldsymbol{\mu}_{M}^{(2)}$, where here $\boldsymbol{\mu}_{M}^{(1)}$ and $\boldsymbol{\mu}_{M}^{(2)}$ are as in (117)-(118) but with $\mathcal{Q}_{M}(\omega)$ replaced by $-\mathcal{L}_{M}(\omega)$. The first integral can be computed by considering the eigen-decomposition of $\mathbf{C}_{M}$ and using the results in Proposition 1. After some algebra, we arrive at

$$
\begin{aligned}
\boldsymbol{\mu}_{M}^{(1)} & =-\frac{1}{2} c_{M} \\
& -\mu_{0} \frac{1}{N} \operatorname{tr}\left[\mathbf{C}_{M}\left(\mathbf{C}_{M}-\mu_{0} \mathbf{I}_{M}\right)^{-1} \odot \mathbf{C}_{M}\left(\mathbf{C}_{M}-\mu_{0} \mathbf{I}_{M}\right)^{-1}\right] \\
& +\frac{\mu_{0}^{2}}{2} \frac{1}{N} \operatorname{tr}\left[\left(\left(\mathbf{C}_{M}-\mu_{0} \mathbf{I}_{M}\right)^{-1} \odot \mathbf{C}_{M}\right) \times\right. \\
& \left.\times\left(\left(\mathbf{C}_{M}-\mu_{0} \mathbf{I}_{M}\right)^{-1} \odot \mathbf{C}_{M}\right)\right] .
\end{aligned}
$$

Let us now deal with the second integral associated with the asymptotic mean, i.e. $\boldsymbol{\mu}_{M}^{(2)}$. To compute this integral, we will follow the approach outlined in Appendix B. We can express $\boldsymbol{\mu}_{M}^{(2)}=\mathcal{J}(1)$, where $\mathcal{J}(x)$ is the following function on $[0,1]$ :

$$
\mathcal{J}(x)=\frac{1}{2 \pi \mathrm{j}} \oint_{\mathcal{C}_{\omega}^{+}} \frac{\frac{1}{N} \operatorname{tr}\left[\mathbf{C}_{M}^{2} \boldsymbol{\Theta}_{M}^{3}(\omega)\right]}{1-\frac{1}{N} \operatorname{tr}\left[\mathbf{C}_{M}^{2} \boldsymbol{\Theta}_{M}^{2}(\omega)\right]} \mathcal{L}_{M}(\omega, x) d \omega
$$

and where $\mathcal{L}_{M}(\omega, x)$ is defined in (105). The value of the function at $x=0$ can be easily computed using the classical residue theorem. Indeed, observe that we can express

$$
\mathcal{J}(0)=\frac{1}{2 \pi \mathrm{j}} \oint_{\mathcal{C}_{\omega}^{+}} \frac{\frac{1}{N} \operatorname{tr}\left[\mathbf{C}_{M}^{2} \boldsymbol{\Theta}_{M}^{3}(\omega)\right]}{1-\frac{1}{N} \operatorname{tr}\left[\mathbf{C}_{M}^{2} \boldsymbol{\Theta}_{M}^{2}(\omega)\right]} \log \left(\omega-\mu_{0}\right) d \omega
$$

and since $\mu_{0}$ is always located outside $\mathcal{C}_{\omega}^{+}, \log \left(\omega-\mu_{0}\right)$ is holomorphic inside the contour. The only poles of the integrand are the eigenvalues $\gamma_{m}, m=1, \ldots, \bar{M}$, and the $2 \bar{M}$ values that null out the denominator, which are denoted by $\vartheta_{m}, m=1, \ldots, 2 \bar{M}$ (counting multiplicities). It is shown above that all these values are inside the integration contour. 
Therefore, computing the residues at these two sets of poles, $1, \ldots, \bar{M}$ we can express $\mathcal{J}(0)$ as

$$
\mathcal{J}(0)=\sum_{m=1}^{\bar{M}} \log \left(\gamma_{m}-\mu_{0}\right)-\frac{1}{2} \sum_{m=1}^{2 \bar{M}} \log \left(\vartheta_{m}-\mu_{0}\right)
$$

On the other hand, according to Lemma 4 in Appendix B, $\mathcal{J}(x)$ is a differentiable function of $x$ on the unit interval, and we can compute its derivative as

$$
\mathcal{J}^{\prime}(x)=\frac{1}{2 \pi \mathrm{j}} \oint_{\mathcal{C}_{\omega}^{+}} \frac{\frac{1}{N} \operatorname{tr}\left[\mathbf{C}_{M}^{2} \boldsymbol{\Theta}_{M}^{3}(\omega)\right]}{1-\frac{1}{N} \operatorname{tr}\left[\mathbf{C}_{M}^{2} \boldsymbol{\Theta}_{M}^{2}(\omega)\right]} \frac{\partial \mathcal{L}_{M}(\omega, x)}{\partial x} d \omega
$$

where

$$
\begin{array}{r}
\frac{\partial \mathcal{L}_{M}(\omega, x)}{\partial x}=-\left(1-\frac{x}{N} \sum_{m=1}^{\bar{M}} \widetilde{K}_{m} \frac{\gamma_{m}}{\gamma_{m}-\omega}\right)^{-1} \times \\
\times \frac{1}{N} \sum_{m=1}^{\bar{M}} \widetilde{K}_{m} \frac{\gamma_{m}}{\gamma_{m}-\omega}
\end{array}
$$

and where $\widetilde{K}_{m}$ is defined in (106). The integral can be solved using conventional residue calculus, taking into account that the only poles are the eigenvalues $\left\{\gamma_{m}\right\}$, the values $\left\{\vartheta_{m}, m=1, \ldots, 2 \bar{M}\right\}$ considered above, and the zeros of the denominator in (127), which are denoted as $\left\{\mu_{m}(x), m=1, \ldots, \bar{M}\right\}$. Computing the corresponding residues, we readily obtain

$$
\begin{aligned}
\mathcal{J}^{\prime}(x) & =\frac{\bar{M}}{x}-\sum_{m=1}^{\bar{M}} \frac{\frac{1}{N} \sum_{r=1}^{\bar{M}} K_{r} \frac{\gamma_{r}^{2}}{1-\frac{1}{N} \sum_{r=1}^{\bar{M}} K_{r} \frac{\gamma_{r}^{2}}{\left(\gamma_{r}-\mu_{m}(x)\right)^{2}}} \mu_{m}^{\prime}(x)}{1 \bar{M} \sum_{m=1}} \frac{\frac{1}{N} \sum_{r=1}^{\bar{M}} \widetilde{K_{r}} \frac{\gamma_{r}}{\gamma_{r}-\vartheta_{m}}}{1-\frac{x}{N} \sum_{r=1}^{\bar{M}} \widetilde{K}_{r} \frac{\gamma_{r}}{\gamma_{r}-\vartheta_{m}}}
\end{aligned}
$$

where $\mu_{m}^{\prime}(x)$ is the derivative of $\mu_{m}(x)$. Finding a primitive of this function and imposing (126) we obtain

$$
\begin{aligned}
\mathcal{J}(x) & =\bar{M} \log x \\
& +\frac{1}{2} \sum_{m=1}^{\bar{M}} \log \left|\frac{K_{m}}{N} \gamma_{m}^{2}\left(1-\frac{1}{N} \sum_{r=1}^{\bar{M}} K_{r} \frac{\gamma_{r}^{2}}{\left(\gamma_{r}-\mu_{m}(x)\right)^{2}}\right)\right| \\
& -\frac{1}{2} \sum_{m=1}^{2 \bar{M}} \log \left|\left(\vartheta_{m}-\mu_{0}\right)\left(1-\frac{x}{N} \sum_{r=1}^{\bar{M}} \widetilde{K_{r}} \frac{\gamma_{r}}{\gamma_{r}-\vartheta_{m}}\right)\right|
\end{aligned}
$$

where we have used (111). The result can be obtained by letting $x \rightarrow 1$ in the above equation. The following lemma will simplify the result.

Lemma 7: The following identities hold for any $r=$

$$
\begin{aligned}
& \prod_{q=1}^{2 \bar{M}}\left(\vartheta_{q}-\gamma_{r}\right)=-\frac{1}{N} K_{r} \gamma_{r}^{2} \prod_{\substack{k=1 \\
k \neq r}}^{\bar{M}}\left(\gamma_{k}-\gamma_{r}\right)^{2} \\
& \prod_{q=1}^{2 \bar{M}}\left(\vartheta_{q}-\mu_{r}\right)=\prod_{k=1}^{\bar{M}}\left(\gamma_{k}-\mu_{r}\right)^{2}\left[1-\frac{1}{N} \sum_{m=1}^{\bar{M}} K_{m} \frac{\gamma_{m}^{2}}{\left(\gamma_{m}-\mu_{r}\right)^{2}}\right] \\
& \prod_{q=1}^{\bar{M}}\left(\mu_{q}-\gamma_{r}\right)=-\frac{K_{r} \gamma_{r}^{2}}{N} \frac{1}{\left(\gamma_{r}-\mu_{0}\right)} \prod_{\substack{k=1 \\
k \neq r}}^{\bar{M}}\left(\gamma_{k}-\gamma_{r}\right) .
\end{aligned}
$$

On the other hand,

$$
\prod_{q=1}^{2 \bar{M}}\left(\vartheta_{q}-\mu_{0}\right)=\prod_{k=1}^{\bar{M}}\left(\gamma_{k}-\mu_{0}\right)^{2}\left[1-\frac{1}{N} \sum_{m=1}^{\bar{M}} K_{m} \frac{\gamma_{m}^{2}}{\left(\gamma_{m}-\mu_{0}\right)^{2}}\right]
$$

and for any $r=1, \ldots, 2 \bar{M}$, we have

$$
\prod_{q=1}^{\bar{M}}\left(\mu_{q}-\vartheta_{r}\right)=\prod_{k=1}^{\bar{M}}\left(\gamma_{k}-\vartheta_{r}\right)\left[1-\frac{1}{N} \sum_{m=1}^{\bar{M}} \frac{\widetilde{K}_{m} \gamma_{m}}{\left(\gamma_{m}-\vartheta_{r}\right)}\right]
$$

Proof: The fact that the quantities $\mu_{q}$ and $\vartheta_{q}$ are roots of polynomial equations allows to identify their associated polynomials as in (108) and (125) respectively. Equations (128), (129) and (131) are directly obtained by evaluating (125) at $\vartheta=\gamma_{r}, \vartheta=\mu_{r}$ and $\vartheta=\mu_{0}$ respectively. On the other hand, (130) and (132) are obtained by evaluating (108) at $\mu=\gamma_{r}$ and $\mu=\vartheta_{r}$ respectively.

Direct application of Lemma 7 allows us to write

$$
\boldsymbol{\mu}_{M}^{(2)}=-\frac{1}{2} \log \left(1-\frac{1}{N} \sum_{m=1}^{\bar{M}} K_{m} \frac{\gamma_{m}^{2}}{\left(\gamma_{m}-\mu_{0}\right)^{2}}\right)
$$

Let us finally compute the asymptotic variance of the GLRT, by direct evaluation of (36), namely

$$
\boldsymbol{\Phi}_{M}=\frac{-1}{4 \pi^{2}} \oint_{\mathcal{C}_{\omega_{1}}^{+}} \oint_{\mathcal{C}_{\omega_{2}}^{+}} \mathcal{L}_{M}\left(\omega_{1}\right) \mathcal{L}_{M}\left(\omega_{2}\right) \bar{\sigma}_{M}^{2}\left(\omega_{1}, \omega_{2}\right) d \omega_{1} d \omega_{2}
$$

where $\bar{\sigma}_{M}^{2}\left(\omega_{1}, \omega_{2}\right)$ is defined in (37) and where we have slightly deformed the contours so that $\mathcal{C}_{\omega_{1}}^{+}$is enclosed by $\mathcal{C}_{\omega_{2}}^{+}$. We will separate $\Phi_{M}$ into three terms, i.e. $\boldsymbol{\Phi}_{M}=\boldsymbol{\Phi}_{M}^{(1)}+$ $\boldsymbol{\Phi}_{M}^{(2)}+\boldsymbol{\Phi}_{M}^{(3)}$, corresponding to the three terms of $\bar{\sigma}_{M}^{2}\left(\omega_{1}, \omega_{2}\right)$ in (37). We will compute these three terms separately. A direct application of Proposition 1 leads to

$$
\begin{aligned}
\boldsymbol{\Phi}_{M}^{(2)} & \triangleq \frac{1+\varsigma}{N} \frac{1}{4 \pi^{2}} \oint_{\mathcal{C}_{\omega_{1}}^{+}} \oint_{\mathcal{C}_{\omega_{2}}^{+}} \mathcal{L}_{M}\left(\omega_{1}\right) \mathcal{L}_{M}\left(\omega_{2}\right) \times \\
& \times \operatorname{tr}\left[\mathbf{C}_{M}^{2} \boldsymbol{\Theta}_{M}^{2}\left(\omega_{1}\right) \boldsymbol{\Theta}_{M}^{2}\left(\omega_{2}\right)\right] d \omega_{1} d \omega_{2} \\
& =-\frac{1+\varsigma}{N} \operatorname{tr}\left[\mathbf{C}_{M}^{2} \boldsymbol{\Theta}_{M}^{2}\left(\mu_{0}\right)\right]
\end{aligned}
$$


and

$$
\begin{aligned}
\boldsymbol{\Phi}_{M}^{(3) \triangleq} & \frac{1+\varsigma}{N} \frac{-1}{4 \pi^{2}} \oint_{\mathcal{C}_{\omega_{1}}^{+}} \oint_{\mathcal{C}_{\omega_{2}}^{+}} \mathcal{L}_{M}\left(\omega_{1}\right) \mathcal{L}_{M}\left(\omega_{2}\right) \times \\
& \times \operatorname{tr}\left[\mathbf{C}_{M} \boldsymbol{\Delta}_{M}\left(\omega_{1}\right) \mathbf{C}_{M} \boldsymbol{\Delta}_{M}\left(\omega_{2}\right)\right] d \omega_{1} d \omega_{2} \\
= & \frac{1+\varsigma}{N} \operatorname{tr}\left[\left(\mathbf{C}_{M}\left(\boldsymbol{\Theta}_{M}\left(\mu_{0}\right)-\operatorname{dg}\left[\mathbf{C}_{M} \boldsymbol{\Theta}_{M}\left(\mu_{0}\right)\right]\right)\right)^{2}\right] .
\end{aligned}
$$

Therefore, it remains to compute

$$
\boldsymbol{\Phi}_{M}^{(1)} \triangleq-\frac{1+\varsigma}{4 \pi^{2}} \oint_{\mathcal{C}_{\omega_{1}}^{+}} \oint_{\mathcal{C}_{\omega_{2}}^{+}} \frac{\mathcal{L}_{M}\left(\omega_{1}\right) \mathcal{L}_{M}\left(\omega_{2}\right)}{\left(\omega_{1}-\omega_{2}\right)^{2}} d \omega_{1} d \omega_{2} .
$$

Note, first of all, that by using the integration by parts formula and classical residue calculus we can write

$$
\begin{aligned}
\frac{1}{2 \pi \mathrm{j}} \oint_{\mathcal{C}_{\omega_{1}}^{+}} \frac{\mathcal{L}_{M}\left(\omega_{1}\right)}{\left(\omega_{1}-\omega_{2}\right)^{2}} d \omega_{1} & =\frac{1}{2 \pi \mathrm{j}} \oint_{\mathcal{C}_{\omega_{1}}^{+}} \frac{1}{\left(\omega_{1}-\omega_{2}\right)} \frac{d \mathcal{L}_{M}\left(\omega_{1}\right)}{d \omega_{1}} d \omega \\
= & -\sum_{m=1}^{M}\left(\frac{1}{\omega_{2}-\mu_{m}}-\frac{1}{\omega_{2}-\gamma_{m}}\right)
\end{aligned}
$$

and therefore, using Proposition 1 together with the identity in (108) evaluated at $\mu=\mu_{0}$, we obtain

$$
\Phi_{M}^{(1)}=-(1+\varsigma) \log \left(1-\frac{1}{N} \sum_{m=1}^{\bar{M}} K_{m} \frac{\gamma_{m}^{2}}{\left(\gamma_{m}-\mu_{0}\right)^{2}}\right) .
$$

1) Boundedness of mean and variance: Let us first analyze the obtained expressions in the undersampled regime. In this situation $c_{M}<1$ and $\mu_{0}=0$, so that the asymptotic mean and variances respectively take the form in (42)-(43). It follows directly from (7) that $\sup _{M}\left|\boldsymbol{\mu}_{M}\right|<\infty$. On the other hand, the function $x \mapsto-x-\log (1-x)$ is monotonically increasing in $[0,1]$ from 0 to $+\infty$. This, together with (7) implies that $\boldsymbol{\Phi}_{M}$ is bounded away from zero for all $M$ (note that the third term is nonnegative). Since the spectral norm of $\mathbf{C}_{M}$ is also bounded, we reach the conclusion that $\boldsymbol{\Phi}_{M}$ is also bounded for all $M$.

The oversampled regime is a bit more complex to analyze, due to the presence of a strictly negative value $\mu_{0}$. This value is the negative solution to

$$
\frac{1}{N} \sum_{m=1}^{\bar{M}} K_{m} \frac{\gamma_{m}}{\gamma_{m}-\mu_{0}}=1 .
$$

Let $\gamma_{\min }$ and $\gamma_{\max }$ denote the minimum and maximum eigenvalues of $\mathbf{C}_{M}$. One can readily establish the fact that

$$
\frac{c_{M}-1}{c_{M}} \gamma_{\min }<\left|\mu_{0}\right|<c_{M} \text {. }
$$

Indeed, the inequality on the right hand side follows from (133) and the fact that $\mu_{0}<0$ and $\operatorname{tr} \mathbf{C}_{M}=M$. The identity on the left hand side is obtained by rewriting (133) as

$$
\begin{aligned}
\frac{c_{M}-1}{c_{M}} & =\frac{1}{M} \sum_{m=1}^{\bar{M}} K_{m} \frac{\left|\mu_{0}\right|}{\gamma_{m}+\left|\mu_{0}\right|} \\
& <\left|\mu_{0}\right| \frac{1}{M} \sum_{m=1}^{\bar{M}} K_{m} \gamma_{m}^{-1} \leq \frac{\left|\mu_{0}\right|}{\gamma_{\min }} .
\end{aligned}
$$

As a consequence of (134) and (As2) - (As3), $\left|\mu_{0}\right|$ is always contained in a compact interval of the positive real axis. On the other hand, we can also establish the bounds

$$
c_{M}^{-1} \leq \frac{1}{N} \operatorname{tr}\left[\mathbf{C}_{M}^{2} \Theta_{M}^{2}\left(\mu_{0}\right)\right]<\frac{\gamma_{\max } c_{M}}{\gamma_{\max } c_{M}+\gamma_{\min }\left(c_{M}-1\right)} .
$$

Indeed, the inequality on the left hand side follows from the application of the Cauchy-Schwarz inequality to (133), whereas the inequality on the right is obtained as

$$
\begin{aligned}
\frac{1}{N} \operatorname{tr}\left[\mathbf{C}_{M}^{2} \Theta_{M}^{2}\left(\mu_{0}\right)\right] & \leq\left(\frac{1}{N} \sum_{m=1}^{\bar{M}} K_{m} \frac{\gamma_{m}}{\gamma_{m}-\mu_{0}}\right) \frac{\gamma_{\max }}{\gamma_{\max }+\left|\mu_{0}\right|} \\
& =\frac{\gamma_{\max }}{\gamma_{\max }+\left|\mu_{0}\right|}
\end{aligned}
$$

together with the lower bound on $\left|\mu_{0}\right|$ established in (134). The bounds in (135) together with (As2) - (As3) imply that this quantity is located in a compact interval inside $(0,1)$ for all $M$. At this point, we have all the ingredients to bound the asymptotic mean and variance of the GLRT statistic.

A trivial use of the triangular inequality and Lemma 1 in (40) shows that

$$
\begin{gathered}
\left|\mu_{M}\right| \leq-\frac{\varsigma}{2} \log \left(1-\frac{1}{N} \operatorname{tr}\left[\mathbf{C}_{M}^{2} \boldsymbol{\Theta}_{M}^{2}\left(\mu_{0}\right)\right]\right) \\
+\frac{1+\varsigma}{2} c_{M}+(1+\varsigma) c_{M}\left(\frac{\left|\mu_{0}\right|^{2}}{2}+1\right)\left|\mu_{0}\right|\left\|\mathbf{C}_{M}\right\|^{2}\left\|\boldsymbol{\Theta}_{M}\left(\mu_{0}\right)\right\|^{2}
\end{gathered}
$$

Since $x \mapsto-\log (1-x)$ is monotonically increasing in $[0,1]$ from 0 to $+\infty$, we see from (135) that the first term is bounded. The second and third terms are bounded thanks to $(\mathbf{A s 2})-(\mathbf{A s 3}),(134)$ and the fact that $\left\|\boldsymbol{\Theta}_{M}\left(\mu_{0}\right)\right\| \leq$ $\left(\gamma_{\min }+\left|\mu_{0}\right|\right)^{-1}$.

Regarding the boundedness of the variance $\Phi_{M}$ in (41), using the fact that $x \mapsto-x-\log (1-x)$ is monotonically increasing in $[0,1]$ from 0 to $+\infty$ and (135) we readily see that $\inf _{M} \Phi_{M}>0$ and that

$\Phi_{M} \leq K+\frac{1+\varsigma}{N} \operatorname{tr}\left[\left(\mathbf{C}_{M}\left(\boldsymbol{\Theta}_{M}\left(\mu_{0}\right)-\operatorname{dg}\left[\mathbf{C}_{M} \boldsymbol{\Theta}_{M}\left(\mu_{0}\right)\right]\right)\right)^{2}\right]$

for some positive constant $K$ independent of $M$. On the other hand, using Lemma 1 together with $($ As2 $)-($ As3 $)$ and $\left\|\boldsymbol{\Theta}_{M}\left(\mu_{0}\right)\right\| \leq\left(\gamma_{\min }+\left|\mu_{0}\right|\right)^{-1}$ we see that the second term above is also bounded for all $M$.

\section{APPENDIX D}

\section{ASYMPTOTIC BEHAVIOR OF QUANTITIES BASED ON THE RESOLVENT}

In this appendix, we follow the convention in Section IV and obviate the dependence on $M$ in all quantities, so $\hat{\mathbf{Q}}_{i}=$ $\hat{\mathbf{Q}}_{M}\left(z_{i}\right), \omega_{i}=\omega_{M}\left(z_{i}\right)$ and so on. We will denote by $\mathbf{A}_{i}$ and $\mathbf{B}_{i}$ certain general deterministic matrices. The $\mathbf{A}_{i}$ 's will be assumed to have bounded norm, whereas for the $\mathbf{B}_{i}$ we will only require that they are Hermitian positive definite and have bounded entries. We recall that the spectral norms of $\mathbf{R}, \overline{\mathbf{Q}}_{i}$, $\hat{\mathbf{R}} \phi, \hat{\mathbf{Q}}_{i} \phi$, etc. are bounded uniformly in $z \in \mathcal{C}$. The following lemma will be constantly used throughout this appendix. 
Lemma 8: Useful identities related to the Schur-Hadamart product:

$$
\begin{gathered}
\operatorname{tr}\left[\mathbf{A}_{1}\left(\mathbf{A}_{2} \odot \mathbf{A}_{3}\right)\right]=\operatorname{tr}\left[\left(\mathbf{A}_{1} \odot \mathbf{A}_{2}^{T}\right) \mathbf{A}_{3}\right] \\
\left(\mathbf{A} \odot \mathbf{B}^{T}\right) \mathbf{1}=\operatorname{vdg}(\mathbf{A B}) \\
\left(\mathbf{a}_{1} \odot \mathbf{a}_{2}\right)\left(\mathbf{a}_{3} \odot \mathbf{a}_{4}\right)^{T}=\left(\mathbf{a}_{1} \mathbf{a}_{3}^{T} \odot \mathbf{a}_{2} \mathbf{a}_{4}^{T}\right)
\end{gathered}
$$

$\operatorname{vdg}\left(\mathbf{A}_{1}\right)^{T}\left(\mathbf{B}_{1} \odot \mathbf{B}_{2}^{T}\right) \operatorname{vdg}\left(\mathbf{A}_{2}\right)=\operatorname{tr}\left[\operatorname{dg}\left(\mathbf{A}_{1}\right) \mathbf{B}_{1} \operatorname{dg}\left(\mathbf{A}_{2}\right) \mathbf{B}_{2}\right]$ $\left(\mathbf{a}_{1} \mathbf{a}_{2}^{T} \odot \mathbf{B}_{1}\right)\left(\mathbf{a}_{3} \mathbf{a}_{4}^{T} \odot \mathbf{B}_{2}\right)=\mathbf{a}_{1} \mathbf{a}_{4}^{T} \odot\left(\mathbf{B}_{1} \operatorname{dg}\left(\mathbf{a}_{3} \mathbf{a}_{2}^{T}\right) \mathbf{B}_{2}\right)$

$$
\begin{aligned}
\operatorname{tr}\left[\left(\mathbf{a}_{1} \mathbf{a}_{2}^{T} \odot \mathbf{B}_{1}\right)\right. & \left.\left(\mathbf{a}_{3} \mathbf{a}_{4}^{T} \odot \mathbf{B}_{2}\right)\right]= \\
& =\left(\mathbf{a}_{1} \odot \mathbf{a}_{4}\right)^{T}\left(\mathbf{B}_{1} \odot \mathbf{B}_{2}^{T}\right)\left(\mathbf{a}_{3} \odot \mathbf{a}_{2}\right) .
\end{aligned}
$$

\section{A. Proof of Proposition 3 (one resolvent)}

Using the first identity in Lemma 8 and observing that $\mathbf{A}_{2}\left(\mathbf{A}_{3} \odot \mathbf{B}_{1}^{T}\right) \mathbf{A}_{1}$ has bounded spectral norm (see Lemma 1 ), we see that it is sufficient to prove the result for the case $\mathbf{A}_{2}=\mathbf{A}_{3}=\mathbf{I}_{M}$ and $\mathbf{B}_{1}=\mathbf{1 1}^{T}$. Let us first concentrate on the expectation of (88). We fist note that we can express the sample covariance matrix $\hat{\mathbf{R}}$ as in (59) where $X_{i j}$ is the $(i, j)$ th entry of $\mathbf{X}$ and where $\mathbf{e}_{i}$ is the $i$ th column of the $M \times M$ identity matrix. Using this, we can write

$$
\mathbb{E}\left[\mathbf{A}_{1} \hat{\mathbf{Q}}_{1} \hat{\mathbf{R}} \phi\right]=\frac{1}{N} \sum_{i=1}^{M} \sum_{j=1}^{N} \mathbb{E}\left[X_{i j} \mathbf{A}_{1} \hat{\mathbf{Q}}_{1} \mathbf{R}^{1 / 2} \mathbf{e}_{i} \mathbf{x}_{j}^{H} \mathbf{R}^{1 / 2} \phi\right] .
$$

Each of the terms of the sum on the right hand side can be developed using the integration by parts formula in (52) of Lemma 2

$$
\begin{aligned}
\mathbb{E}\left[\mathbf{A}_{1} \hat{\mathbf{Q}}_{1} \hat{\mathbf{R}} \phi\right] & =\mathbb{E}\left[\mathbf{A}_{1} \hat{\mathbf{Q}}_{1} \mathbf{R} \phi\right] \\
+ & z \frac{1+\varsigma}{N} \mathbb{E}\left[\mathbf{A}_{1} \hat{\mathbf{Q}}_{1} \operatorname{dg}\left(\hat{\mathbf{Q}}_{1} \mathbf{R}\right) \hat{\mathbf{R}} \phi\right] \\
& -\mathbb{E}\left[\left(\mathbf{A}_{1} \hat{\mathbf{Q}}_{1} \hat{\mathbf{R}}\right) \frac{1}{N} \operatorname{tr}\left[\hat{\mathbf{Q}}_{1} \mathbf{R}\right] \phi\right] \\
& -\varsigma \frac{1}{N} \mathbb{E}\left[\mathbf{A}_{1} \hat{\mathbf{Q}}_{1} \mathbf{R} \hat{\mathbf{Q}}_{1} \hat{\mathbf{R}} \phi\right]+\mathcal{O}\left(N^{-\mathbb{N}}\right) .
\end{aligned}
$$

Using the definition of $\beta_{1}=\beta\left(z_{1}\right)$ in (58) we can express the above equation as

$$
\begin{aligned}
& \left(1+\frac{1}{N} \operatorname{tr}\left[\overline{\mathbf{Q}}_{1} \mathbf{R}\right]\right) \mathbb{E}\left[\mathbf{A}_{1} \hat{\mathbf{Q}}_{1} \hat{\mathbf{R}} \phi\right]= \\
& =\mathbb{E}\left[\mathbf{A}_{1} \hat{\mathbf{Q}}_{1} \mathbf{R} \phi\right]+z \frac{1+\varsigma}{N} \mathbb{E}\left[\mathbf{A}_{1} \hat{\mathbf{Q}}_{1} \operatorname{dg}\left(\hat{\mathbf{Q}}_{1} \mathbf{R}\right) \hat{\mathbf{R}} \phi\right] \\
& -\frac{1}{N} \mathbb{E}\left[\left(\mathbf{A}_{1} \hat{\mathbf{Q}}_{1} \hat{\mathbf{R}} \phi\right) \beta_{1}\right]-\varsigma \frac{1}{N} \mathbb{E}\left[\mathbf{A}_{1} \hat{\mathbf{Q}}_{1} \mathbf{R} \hat{\mathbf{Q}}_{1} \hat{\mathbf{R}} \phi\right]+\mathcal{O}\left(N^{-\mathbb{N}}\right) .
\end{aligned}
$$

Now, using the identity in (69) together with the lower bound on (70) we see that we can write

$$
\begin{gathered}
\mathbb{E}\left[\widetilde{\boldsymbol{\digamma}}_{1}\left(z_{1}\right)\right]=\frac{1}{N} \mathbb{E} \operatorname{tr}\left[\mathbf{A}_{1} \hat{\mathbf{Q}}_{1} \hat{\mathbf{R}} \phi\right] \\
=\frac{z_{1}}{\omega_{1}} \frac{1}{N} \mathbb{E} \operatorname{tr}\left[\mathbf{A}_{1} \hat{\mathbf{Q}}_{1} \mathbf{R} \phi\right]+\frac{z_{1}^{2}}{\omega_{1}} \frac{1+\varsigma}{N^{2}} \mathbb{E} \operatorname{tr}\left[\mathbf{A}_{1} \hat{\mathbf{Q}}_{1} \operatorname{dg}\left(\hat{\mathbf{Q}}_{1} \mathbf{R}\right) \hat{\mathbf{R}} \phi\right] \\
-\frac{z_{1}}{\omega_{1}} \mathbb{E}\left[\frac{1}{N} \operatorname{tr}\left[\mathbf{A}_{1} \hat{\mathbf{Q}}_{1} \hat{\mathbf{R}} \phi\right] \frac{\beta_{1}}{N}\right]-\frac{z_{1}}{\omega_{1}} \varsigma \frac{1}{N^{2}} \mathbb{E} \operatorname{tr}\left[\begin{array}{c}
\left.\mathbf{A}_{1} \hat{\mathbf{Q}}_{1} \mathbf{R} \hat{\mathbf{Q}}_{1} \hat{\mathbf{R}} \phi\right] \\
+\mathcal{O}\left(N^{-\mathbb{N}}\right) .
\end{array}\right.
\end{gathered}
$$

Using Lemma 1, the second and fourth terms on the right hand side of the above equation can be easily bounded by quantity of type $\mathcal{O}\left(N^{-1}\right)$, so that

$$
\begin{aligned}
\mathbb{E}\left[\widetilde{\digamma}_{1}\left(z_{1}\right)\right] & =\frac{z_{1}}{\omega_{1}} \frac{1}{N} \mathbb{E} \operatorname{tr}\left[\mathbf{A}_{1} \hat{\mathbf{Q}}_{1} \mathbf{R} \phi\right] \\
& -\frac{z_{1}}{\omega_{1}} \mathbb{E}\left[\frac{1}{N} \operatorname{tr}\left[\mathbf{A}_{1} \hat{\mathbf{Q}}_{1} \hat{\mathbf{R}} \phi\right] \frac{\beta_{1}}{N}\right]+\mathcal{O}\left(N^{-1}\right) .
\end{aligned}
$$

Next, consider next the quantity $\mathbb{E}\left[\mathbf{A}_{1} \hat{\mathbf{Q}}_{1} \hat{\mathbf{D}}\right]$. Using the fact that $\hat{\mathbf{D}}=\operatorname{dg}(\hat{\mathbf{R}})$, inserting the expression of $\hat{\mathbf{R}}$ (59) and applying again the integration by parts formula (52) in Lemma 2 , we can similarly write

$$
\begin{aligned}
\frac{1}{N} \mathbb{E} \operatorname{tr} & {\left[\mathbf{A}_{1} \hat{\mathbf{Q}}_{1} \hat{\mathbf{D}} \phi\right]=\frac{1}{N} \mathbb{E} \operatorname{tr}\left[\mathbf{A}_{1} \hat{\mathbf{Q}}_{1} \mathbf{D} \phi\right] } \\
& +z_{1} \frac{1+\varsigma}{N^{2}} \mathbb{E} \operatorname{tr}\left[\mathbf{A}_{1} \hat{\mathbf{Q}}_{1}\left(\hat{\mathbf{Q}}_{1} \odot \mathbf{R}^{T} \odot \hat{\mathbf{R}}\right) \phi\right] \\
& -\frac{1}{N^{2}} \mathbb{E} \operatorname{tr}\left[\mathbf{A}_{1} \hat{\mathbf{Q}}_{1} \hat{\mathbf{R}} \operatorname{dg}\left(\mathbf{R}_{\mathbf{Q}_{1}}\right) \phi\right] \\
& -\varsigma \frac{1}{N^{2}} \mathbb{E} \operatorname{tr}\left[\mathbf{A}_{1} \hat{\mathbf{Q}}_{1} \mathbf{R} \operatorname{dg}\left(\hat{\mathbf{R}} \hat{\mathbf{Q}}_{1}\right) \phi\right]+\mathcal{O}\left(N^{-\mathbb{N}}\right) .
\end{aligned}
$$

Using here again Lemma 1 we readily see that

$$
\frac{1}{N} \mathbb{E} \operatorname{tr}\left[\mathbf{A}_{1} \hat{\mathbf{Q}}_{1} \hat{\mathbf{D}} \phi\right]=\frac{1}{N} \mathbb{E} \operatorname{tr}\left[\mathbf{A}_{1} \hat{\mathbf{Q}}_{1} \mathbf{D} \phi\right]+\mathcal{O}\left(N^{-1}\right) .
$$

Hence, combining (139) and (138) we obtain

$$
\begin{aligned}
& \frac{1}{N} \mathbb{E} \operatorname{tr}\left[\mathbf{A}_{1}\left(\hat{\mathbf{Q}}_{1} \phi-\overline{\mathbf{Q}}_{1}\right)\right]= \\
& \quad=\frac{z_{1}}{\omega_{1}} \mathbb{E}\left[\frac{1}{N} \operatorname{tr}\left[\mathbf{A}_{1} \hat{\mathbf{Q}}_{1} \hat{\mathbf{R}} \overline{\mathbf{Q}}_{1} \phi\right] \frac{\beta_{1}}{N}\right]+\mathcal{O}\left(N^{-1}\right) .
\end{aligned}
$$

Assume that we have proven that $\operatorname{var} \digamma_{1}\left(z_{1}\right)=\mathcal{O}\left(N^{-2}\right)$, so that in particular $\operatorname{var}\left(\beta_{1} / N\right)=\mathcal{O}\left(N^{-2}\right)$. This will imply

$$
\begin{aligned}
& \frac{1}{N} \mathbb{E} \operatorname{tr}\left[\mathbf{A}_{1}\left(\hat{\mathbf{Q}}_{1} \phi-\overline{\mathbf{Q}}_{1}\right)\right]= \\
& =\frac{z_{1}}{\omega_{1}} \mathbb{E}\left[\frac{1}{N} \operatorname{tr}\left[\mathbf{A}_{1} \hat{\mathbf{Q}}_{1} \hat{\mathbf{R}} \overline{\mathbf{Q}}_{1} \phi\right]\right] \mathbb{E}\left[\frac{\beta_{1}}{N}\right]+\mathcal{O}\left(N^{-1}\right)
\end{aligned}
$$

and the result will follow from the fact that $\mathbb{E}\left[N^{-1} \beta_{1}\right]=$ $\mathcal{O}\left(N^{-1}\right)$. To see this last point, define $\widetilde{\mathbf{Q}}_{1}=\widetilde{\mathbf{Q}}\left(z_{1}\right)$, where

$$
\widetilde{\mathbf{Q}}(z)=(\hat{\mathbf{R}}-z \mathbf{D})^{-1}
$$

and note that, similarly to (49), we can establish the bound

$$
\|\widetilde{\mathbf{Q}}(z)\| \leq\left\|\mathbf{D}^{-1}\right\|\left\|\left(\widetilde{\mathbf{C}}-z \mathbf{I}_{M}\right)^{-1}\right\|<\frac{K}{\operatorname{dist}(z, \mathcal{T})}
$$

for some positive $K>0$ independent of $N$. On the other hand, we can also establish the identity

$$
\hat{\mathbf{Q}}_{1}-\widetilde{\mathbf{Q}}_{1}=z_{1} \widetilde{\mathbf{Q}}_{1}(\hat{\mathbf{D}}-\mathbf{D}) \hat{\mathbf{Q}}_{1}
$$

and this implies that

$$
\begin{aligned}
& \mathbb{E}\left[\frac{\beta_{1}}{N}\right]=\frac{1}{N} \mathbb{E} \operatorname{tr}\left[\mathbf{R}\left(\widetilde{\mathbf{Q}}_{1} \phi-\overline{\mathbf{Q}}_{1}\right)\right] \\
&+z_{1} \frac{1}{N} \mathbb{E} \operatorname{tr}\left[\hat{\mathbf{Q}}_{1} \mathbf{R} \widetilde{\mathbf{Q}}_{1}(\hat{\mathbf{D}}-\mathbf{D}) \phi\right]
\end{aligned}
$$


Now, from well known results of the sample covariance matrix, the first term is well known to be expressible as $\mathcal{O}\left(N^{-1}\right)$ [37] As for the second term, we can use the fact that

$$
\begin{aligned}
\hat{\mathbf{D}} & =\operatorname{dg} \hat{\mathbf{R}}=\sum_{m=1}^{M} \mathbf{e}_{m} \mathbf{e}_{m}^{T} \hat{\mathbf{R}} \mathbf{e}_{m} \mathbf{e}_{m}^{T} \\
& =\frac{1}{N} \sum_{i=1}^{M} \sum_{j=1}^{N} \sum_{m=1}^{M} \mathbf{e}_{m} \mathbf{e}_{m}^{T} X_{i j} \mathbf{R}^{1 / 2} \mathbf{e}_{i} \mathbf{x}_{j}^{H} \mathbf{R}^{1 / 2} \mathbf{e}_{m} \mathbf{e}_{m}^{T}
\end{aligned}
$$

where in the second identity we have inserted the expansion of $\hat{\mathbf{R}}$ in (59). Inserting the above equation into the second term on the right hand side of (143) and applying the integration by parts formula (52) in Lemma 2 together with the bounds in Lemma 1 and (49)-(142) we can obtain

$$
\frac{1}{N} \mathbb{E} \operatorname{tr}\left[\hat{\mathbf{Q}}_{1} \mathbf{R} \widetilde{\mathbf{Q}}_{1}(\hat{\mathbf{D}}-\mathbf{D}) \phi\right]=\mathcal{O}\left(N^{-1}\right) .
$$

From (143) we conclude that $\mathbb{E}\left[\beta_{1} / N\right]=\mathcal{O}\left(N^{-1}\right)$ as we wanted to prove. Using this fact in (141) directly proves (89). As for (90), it follows directly from the application of (89) into (138), together with the fact that $\mathbb{E}\left[N^{-1} \beta_{1}\right]=\mathcal{O}\left(N^{-1}\right)$ and $\operatorname{var}\left(\beta_{1} / N\right)=\mathcal{O}\left(N^{-2}\right)$ (note that this last fact is still to be shown).

To conclude the prove of Proposition 3, it remains to see that the variance of the two random quantities under analysis are $\mathcal{O}\left(N^{-2}\right)$. In particular, this will prove the fact $\operatorname{var}\left(\beta_{1} / N\right)=\mathcal{O}\left(N^{-2}\right)$, which was needed in the asymptotic characterization of the above expectations. To see this, we will directly apply the Poincaré-Nash inequality presented in (53) of Proposition 2. In particular, we observe that we can write

$$
\begin{array}{rl}
\operatorname{var}\left[\widetilde{\digamma}_{1}\left(z_{1}\right)\right] \leq \sum_{i=1}^{M} \sum_{j=1}^{N} & \mathbb{E}\left|\frac{\partial \frac{1}{N} \operatorname{tr}\left[\mathbf{A}_{1} \hat{\mathbf{Q}}_{1} \hat{\mathbf{R}} \phi\right]}{\partial X_{i j}}\right|^{2} \\
+(1-\varsigma) \mathbb{E}\left|\frac{\partial \frac{1}{N} \operatorname{tr}\left[\mathbf{A}_{1} \hat{\mathbf{Q}}_{1} \hat{\mathbf{R}} \phi\right]}{\partial X_{i j}^{*}}\right|^{2}
\end{array}
$$

whereas an equivalent expression is valid for var $\left[\digamma_{1}\left(z_{1}\right)\right]$. The following lemma together with the bound in (49) allows us to conclude that these variances are all $\mathcal{O}\left(N^{-2}\right)$.

Lemma 9: Let $\mathbf{\Upsilon}=\mathbf{\Upsilon}\left(\mathbf{X}, \mathbf{X}^{*}, z_{1}, z_{2}\right)$ be an observationdependent random matrix such that $\sup _{M}\|\mathbf{\Upsilon} \phi\|<\infty$ uniformly on $\left(z_{1}, z_{2}\right) \in \mathcal{C} \times \mathcal{C}$. Then, we can write

$$
\begin{aligned}
\sum_{i=1}^{M} \sum_{j=1}^{N} \mathbb{E}\left|\frac{1}{N} \operatorname{tr}\left[\mathbf{\Upsilon} \frac{\partial \hat{\mathbf{R}}}{\partial X_{i j}^{*}} \phi\right]\right|^{2} & =\mathcal{O}\left(N^{-2}\right) \\
\sum_{i=1}^{M} \sum_{j=1}^{N} \mathbb{E}\left|\frac{1}{N} \operatorname{tr}\left[\mathbf{\Upsilon} \frac{\partial \hat{\mathbf{Q}}_{1}}{\partial X_{i j}^{*}} \phi\right]\right|^{2} & =\mathcal{O}\left(N^{-2}\right) \\
\sum_{i=1}^{M} \sum_{j=1}^{N} \mathbb{E}\left|\frac{\partial \phi}{\partial X_{i j}^{*}}\right|^{2} & =\mathcal{O}\left(N^{-\mathbb{N}}\right)
\end{aligned}
$$

Furthermore, the same identities hold when $\partial(\cdot) / \partial X_{i j}^{*}$ is replaced by $\partial(\cdot) / \partial X_{i j}$ in all the expressions above.
Proof: To prove the first identity, we simply use (67) in combination with Jensen's inequality and Lemma 1, so that

$$
\begin{aligned}
\sum_{i=1}^{M} \sum_{j=1}^{N} \mathbb{E} \mid \frac{1}{N} \operatorname{tr} & {\left.\left[\boldsymbol{\Upsilon} \frac{\partial \hat{\mathbf{R}}}{\partial X_{i j}^{*}} \phi\right]\right|^{2} \leq \frac{2}{N^{2}} \frac{1}{N} \mathbb{E} \operatorname{tr}\left[\mathbf{\Upsilon} \hat{\mathbf{R}} \Upsilon^{H} \mathbf{R} \phi\right] } \\
+ & \varsigma \frac{2}{N^{2}} \frac{1}{N} \mathbb{E} \operatorname{tr}\left[\mathbf{\Upsilon}^{H} \hat{\mathbf{R}} \boldsymbol{\Upsilon} \mathbf{R} \phi\right]=\mathcal{O}\left(N^{-2}\right) .
\end{aligned}
$$

Regarding the second identity, we use (68) also together with Jensen's inequality and Lemma 1, namely

$$
\begin{aligned}
& \sum_{i=1}^{M} \sum_{j=1}^{N} \mathbb{E}\left|\frac{1}{N} \operatorname{tr}\left[\mathbf{\Upsilon} \frac{\partial \hat{\mathbf{Q}}_{1}}{\partial X_{i j}^{*}} \phi\right]\right|^{2} \leq \\
& \leq 4 \frac{\left|z_{1}\right|^{2}}{N^{2}} \frac{1}{N} \mathbb{E} \operatorname{tr}\left[\mathbf{R} \operatorname{dg}\left(\hat{\mathbf{Q}}_{1} \mathbf{\Upsilon} \hat{\mathbf{Q}}_{1}\right) \hat{\mathbf{R}} \operatorname{dg}\left(\hat{\mathbf{Q}}_{1} \mathbf{\Upsilon} \hat{\mathbf{Q}}_{1}\right)^{H} \phi\right] \\
& +4 \varsigma \frac{\left|z_{1}\right|^{2}}{N^{2}} \frac{1}{N} \mathbb{E} \operatorname{tr}\left[\mathbf{R} \operatorname{dg}\left(\hat{\mathbf{Q}}_{1} \mathbf{\Upsilon} \hat{\mathbf{Q}}_{1}\right)^{H} \hat{\mathbf{R}} \operatorname{dg}\left(\hat{\mathbf{Q}}_{1} \mathbf{\Upsilon} \hat{\mathbf{Q}}_{1}\right) \phi\right] \\
& +4 \frac{1}{N^{2}} \frac{1}{N} \mathbb{E} \operatorname{tr}\left[\left(\hat{\mathbf{Q}}_{1} \mathbf{\Upsilon} \hat{\mathbf{Q}}_{1}\right) \hat{\mathbf{R}}\left(\hat{\mathbf{Q}}_{1} \mathbf{\Upsilon} \hat{\mathbf{Q}}_{1}\right)^{H} \mathbf{R} \phi\right] \\
& +4 \varsigma \frac{1}{N^{2}} \frac{1}{N} \mathbb{E} \operatorname{tr}\left[\left(\hat{\mathbf{Q}}_{1} \mathbf{\Upsilon} \hat{\mathbf{Q}}_{1}\right) \mathbf{R}\left(\hat{\mathbf{Q}}_{1} \mathbf{\Upsilon} \hat{\mathbf{Q}}_{1}\right)^{H} \hat{\mathbf{R}} \phi\right]=\mathcal{O}\left(N^{-2}\right) .
\end{aligned}
$$

Regarding the last identity in the statement of the lemma, we know from Appendix E that

$$
\frac{\partial \phi_{M}}{\partial X_{i j}^{*}}=\frac{1+\varsigma}{N} \mathbf{e}_{i}^{T} \mathbf{R}^{1 / 2} \operatorname{adj}(\phi(\hat{\mathbf{R}})) \phi^{\prime}(\hat{\mathbf{R}}) \mathbf{R}^{1 / 2} \mathbf{x}_{j}
$$

where $\operatorname{adj}(\mathbf{A})$ is the adjugate of $\mathbf{A}$. Therefore,

$$
\begin{aligned}
& \sum_{i=1}^{M} \sum_{j=1}^{N} \mathbb{E}\left|\frac{\partial \phi}{\partial X_{i j}^{*}}\right|^{2} \\
& =\frac{(1+\varsigma)^{2}}{N} \operatorname{tr}\left[\mathbf{R} \hat{\mathbf{R}}\left(\phi^{\prime}(\hat{\mathbf{R}}) \operatorname{adj}(\phi(\hat{\mathbf{R}}))\right)^{2}\right]
\end{aligned}
$$

and the quantity on the right hand side is shown to be $\mathcal{O}\left(N^{-\mathbb{N}}\right)$ in Appendix $\mathrm{E}$. The proofs for the case where $\partial() / \partial X_{i j}^{*}$ is replaced by $\partial() / \partial X_{i j}$ are completely equivalent and are therefore omitted.

\section{B. Proof of Proposition 4 (two resolvents)}

We define the following matrices, which will be useful in the algebraic development of this section:

$$
\begin{array}{r}
\Pi_{M}=\operatorname{dgvec}\left(\mathbf{I}_{M}\right)=\sum_{r=1}^{M}\left(\mathbf{e}_{r} \mathbf{e}_{r}^{T} \otimes \mathbf{e}_{r} \mathbf{e}_{r}^{T}\right) \\
\tilde{\Pi}_{M, N}=\sum_{i=1}^{M} \sum_{j=1}^{N}\left(\tilde{\mathbf{e}}_{j} \mathbf{e}_{i}^{T} \otimes \mathbf{e}_{i} \tilde{\mathbf{e}}_{j}^{T}\right)
\end{array}
$$

where $\mathbf{e}_{i}$ (respectively $\tilde{\mathbf{e}}_{j}$ ) denotes the $i$ th (respectively $j$ th) column of the $M \times M$ (respectively $N \times N$ ) identity matrix, and where $\operatorname{dgvec}(\mathbf{A})$ denotes a diagonal matrix with diagonal entries equal to $\operatorname{vec}(\mathbf{A})$. We will also write $\tilde{\Pi}_{M}=\tilde{\Pi}_{M, M}$. The above matrices have very interesting properties related to the Kronecker and Hadamard products, that are summarized in what follows. 
Remark 3: The $M^{2} \times M^{2}$ matrix $\Pi_{M}$ is idempotent and can be decomposed as $\Pi_{M}=\mathbf{W}_{M} \mathbf{W}_{M}^{H}$, where the $M^{2} \times M$ matrix $\mathbf{W}_{M}$ is defined as

$$
\mathbf{W}_{M}=\left[\mathbf{e}_{1} \mathbf{e}_{1}^{T}, \ldots, \mathbf{e}_{M} \mathbf{e}_{M}^{T}\right]^{T}
$$

Furthermore, it holds that [41] $\mathbf{W}_{M}^{H}(\mathbf{A} \otimes \mathbf{B}) \mathbf{W}_{M}=$ $(\mathbf{A} \odot \mathbf{B})$ for any two $M \times M$ matrices $\mathbf{A}$ and $\mathbf{B}$. The $M N \times M N$ permutation matrix $\tilde{\Pi}_{M, N}$ is sometimes referred to as the Kronecker commutation matrix. Indeed, if $\mathbf{A}$ is an $N \times M$ matrix and $\mathbf{B}$ is an $M \times N$ matrix, we have $\tilde{\Pi}_{M, N}^{T}(\mathbf{A} \otimes \mathbf{B}) \tilde{\Pi}_{M, N}=\mathbf{B} \otimes \mathbf{A}$. It holds that $\mathbf{W}_{M}^{H} \tilde{\Pi}_{M}=$ $\mathbf{W}_{M}^{H}$, so that $\Pi_{M} \tilde{\Pi}_{M}=\Pi_{M}$.

The following two lemmas will be repeatedly used in this subsection:

Lemma 10: Let $\mathbf{A}_{1}, \ldots, \mathbf{A}_{8}$, and $\mathbf{B}_{1}, \ldots, \mathbf{B}_{3}$ be all $M \times M$ matrices. Then, the identities in (146)-(147) at the top of next page hold true, where $\Pi_{M}$ is as defined in (144).

Proof: To prove the first result, we begin by using the fact that, for four matrices of appropriate dimensions $\mathbf{A}_{1} \ldots \mathbf{A}_{4}$, one can write $\operatorname{tr}\left[\mathbf{A}_{1} \mathbf{A}_{2} \mathbf{A}_{3} \mathbf{A}_{4}\right]=$ $\operatorname{vec}\left(\mathbf{A}_{4}^{T}\right)^{T}\left(\mathbf{A}_{3}^{T} \otimes \mathbf{A}_{1}\right) \operatorname{vec}\left(\mathbf{A}_{2}\right)$, so that, using $\operatorname{vec}\left(\mathbf{a}_{1} \mathbf{a}_{2}^{T}\right)=$ $\mathbf{a}_{2} \otimes \mathbf{a}_{1}$ and noticing that $\mathbf{a}_{1} \odot \mathbf{a}_{2}=\operatorname{dgvec}\left(\mathbf{a}_{1}\right) \mathbf{a}_{2}$ we obtain the result. The second identity is proved similarly.

Lemma 11: Let $\mathbf{A}_{1}, \ldots, \mathbf{A}_{8}$, and $\mathbf{B}_{1}, \ldots, \mathbf{B}_{3}$ be matrices of appropriate dimensions. Denote by $\tilde{\mathbf{e}}_{j}$ the $j$ th column vector of the $N \times N$ identity matrix (as opposed to $\mathbf{e}_{i}$, that denotes the $i$ th column vector of the $M \times M$ identity matrix). Then,

$$
\begin{gathered}
\sum_{i=1}^{M} \sum_{j=1}^{N} \operatorname{tr}\left[\mathbf{A}_{1}\left(\mathbf{A}_{2} \mathbf{e}_{i} \tilde{\mathbf{e}}_{j}^{T} \mathbf{A}_{3} \odot \mathbf{B}_{1}\right) \mathbf{A}_{4}\left(\mathbf{A}_{5} \tilde{\mathbf{e}}_{j} \mathbf{e}_{i}^{T} \mathbf{A}_{6} \odot \mathbf{B}_{2}\right)\right] \\
=\operatorname{tr}\left[\left(\mathbf{A}_{1} \odot\left(\mathbf{A}_{2} \mathbf{A}_{6}\right)^{T}\right) \mathbf{B}_{1}\left(\mathbf{A}_{4} \odot\left(\mathbf{A}_{5} \mathbf{A}_{3}\right)^{T}\right) \mathbf{B}_{2}\right]
\end{gathered}
$$

and the identites in (148)-(149)-(150)] at the top of next page hold true, where $\tilde{\Pi}_{M}$ is the Kronecker commutation matrix defined in (144).

Proof: Very similar to the proof of Lemma 10, and therefore omitted. The only interesting point is the simplification that is applied to the first equation, which is a consequence of the identities $\operatorname{tr}\left[\mathbf{A}_{1} \operatorname{dgvec}\left(\mathbf{B}_{1}\right) \mathbf{A}_{2} \operatorname{dgvec}\left(\mathbf{B}_{2}\right)\right]=$ $\operatorname{vec}\left(\mathbf{B}_{1}\right)^{T}\left(\mathbf{A}_{2} \odot \mathbf{A}_{1}^{T}\right) \operatorname{vec}\left(\mathbf{B}_{2}\right)$ and $\operatorname{vec}\left(\mathbf{B}_{1}\right)^{T}\left(\mathbf{A}_{1} \otimes \mathbf{A}_{2}\right) \operatorname{vec}\left(\mathbf{B}_{2}\right)=\operatorname{tr}\left[\mathbf{A}_{1}^{T} \mathbf{B}_{1}^{T} \mathbf{A}_{2} \mathbf{B}_{2}\right]$, valid for matrices of appropriate dimensions.

Having introduced these analytical tools, let us focus on the proof of Proposition 4. The fact that $\operatorname{var} \digamma_{2}\left(z_{1}, z_{2}\right)$ and $\operatorname{var} \widetilde{\boldsymbol{F}}_{2}\left(z_{1}, z_{2}\right)$ are both $\mathcal{O}\left(N^{-2}\right)$ follows directly from the Poincaré-Nash inequality in (53) of Proposition 2 together with Lemma 9 presented above. Indeed, observe that both $\partial \widetilde{\boldsymbol{F}}_{2}\left(z_{1}, z_{2}\right) / \partial X_{i j}$ and $\partial \widetilde{\boldsymbol{F}}_{2}\left(z_{1}, z_{2}\right) / \partial X_{i j}^{*}$ can be expressed as a sum of terms of the form given in the statement of Lemma
9, for example

$$
\begin{aligned}
& \frac{\partial \widetilde{\boldsymbol{\digamma}}_{2}\left(z_{1}, z_{2}\right)}{\partial X_{i j}} \\
& =\frac{1}{N} \mathbb{E} \operatorname{tr}\left[\mathbf{E}_{1} \frac{\partial \hat{\mathbf{Q}}_{1}}{\partial X_{i j}} \hat{\mathbf{R}} \phi\right]+\frac{1}{N} \mathbb{E} \operatorname{tr}\left[\mathbf{E}_{1} \hat{\mathbf{Q}}_{1} \frac{\partial \hat{\mathbf{R}}}{\partial X_{i j}} \phi\right] \\
& +\frac{1}{N} \mathbb{E} \operatorname{tr}\left[\mathbf{E}_{2} \frac{\partial \hat{\mathbf{Q}}_{2}}{\partial X_{i j}} \phi\right]+\frac{1}{N} \mathbb{E} \operatorname{tr}\left[\mathbf{E}_{2} \hat{\mathbf{Q}}_{2} \phi\right] \frac{\partial \phi}{\partial X_{i j}} \\
& +\mathcal{O}\left(N^{-\mathbb{N}}\right) .
\end{aligned}
$$

where we have defined

$$
\begin{aligned}
& \mathbf{E}_{1}=\mathbf{A}_{3}\left(\mathbf{A}_{4}\left(\mathbf{A}_{5} \hat{\mathbf{Q}}_{2} \mathbf{A}_{6} \odot \mathbf{B}_{2}\right) \mathbf{A}_{1} \odot \mathbf{B}_{1}^{T}\right) \mathbf{A}_{2} \hat{\mathbf{Q}}_{1} \\
& \mathbf{E}_{2}=\mathbf{A}_{6}\left(\mathbf{A}_{1}\left(\mathbf{A}_{2} \hat{\mathbf{Q}}_{1} \hat{\mathbf{R}} \mathbf{A}_{3} \odot \mathbf{B}_{1}\right) \mathbf{A}_{4} \odot \mathbf{B}_{2}^{T}\right) \mathbf{A}_{5}
\end{aligned}
$$

and where we basically applied the property in (136) of Lemma 8. Using Lemma 1 we can readily check that the matrices multiplying the matrix derivatives inside the traces have uniformly bounded norm. Therefore, application of the Jensen's inequality together with Lemma 9 shows that $\operatorname{var} \widetilde{\digamma}_{2}\left(z_{1}, z_{2}\right)=\mathcal{O}\left(N^{-2}\right)$. The same reasoning applies to $\operatorname{var} \digamma_{2}\left(z_{1}, z_{2}\right)$.

Let us next draw our attention to the expectation of the two functions $\digamma_{2}\left(z_{1}, z_{2}\right)$ and $\widetilde{\digamma}_{2}\left(z_{1}, z_{2}\right)$. Replacing $\hat{\mathbf{R}}$ in the definition of $\widetilde{\digamma}_{2}\left(z_{1}, z_{2}\right)$ by its decomposition in (59) and using the integration by parts formula (52) in Lemma 2 we obtain, applying several algebraic identities in Lemma 8, $\mathbb{E}\left[\widetilde{\digamma}_{2}\left(z_{1}, z_{2}\right)\right]$ as in (153) at the top of the next page, where $\beta_{1}$ is defined in (58), $\widetilde{\chi}_{1}\left(z_{1}, z_{2}\right)$ is as in (154) at the top of the next page, and where

$$
\begin{aligned}
\tilde{\chi}_{2}\left(z_{1}, z_{2}\right)=-\frac{z_{1}}{\omega_{1}} \mathbb{E}\left[\frac{1}{N} \operatorname{tr}\right. & {\left[\mathbf{A}_{1}\left(\mathbf{A}_{2} \hat{\mathbf{Q}}_{1} \hat{\mathbf{R}} \mathbf{A}_{3} \odot \mathbf{B}_{1}\right) \times\right.} \\
& \left.\left.\times \mathbf{A}_{4}\left(\mathbf{A}_{5} \hat{\mathbf{Q}}_{2} \mathbf{A}_{6} \odot \mathbf{B}_{2}\right)\right] \beta_{1}\right] .
\end{aligned}
$$

Note here that matrices of the type $\operatorname{dgvec}\left(\mathbf{B}_{1}\right)$ have uniformly bounded spectral norm thanks to the bounded entries of $\mathbf{B}_{1}$. Therefore, applying the bounds in Lemma 1 together with the fact that $\operatorname{tr} \Pi_{M}=\operatorname{tr} \tilde{\Pi}_{M}=M$ and $\tilde{\Pi}_{M}=\tilde{\Pi}_{M}^{T}$, we obtain $\tilde{\chi}_{1}\left(z_{1}, z_{2}\right)=\mathcal{O}\left(N^{-1}\right)$. On the other hand, the fact that $\mathbb{E} \beta_{1}=\mathcal{O}\left(N^{-1}\right)$ and $\operatorname{var} \beta_{1}=\mathcal{O}\left(N^{-2}\right)$ (Proposition 3) implies $\mathbb{E}\left|\beta_{1}\right|^{2}=\mathcal{O}\left(N^{-2}\right)$. Thus, the application of the Cauchy-Schwarz directly shows that $\widetilde{\chi}_{2}\left(z_{1}, z_{2}\right)=\mathcal{O}\left(N^{-1}\right)$.

Going back to (153), we observe that the first term on the right hand is similar to $\mathbb{E}\left[\digamma_{2}\left(z_{1}, z_{2}\right)\right]$. In order to get around this dependency, we analyze next a quantity very similar to $\widetilde{\digamma}_{2}\left(z_{1}, z_{2}\right)$ but replacing $\hat{\mathbf{R}}$ by the diagonal $\hat{\mathbf{D}}$. Using $\hat{\mathbf{D}}=\operatorname{dg}(\hat{\mathbf{R}})$ together with the fact that $\hat{\mathbf{R}}$ can be decomposed as in (59) and applying the integration by parts formula (52) in Lemma 2, we are able to write

$$
\begin{array}{r}
\frac{1}{N} \mathbb{E} \operatorname{tr}\left[\mathbf{A}_{1}\left(\mathbf{A}_{2} \hat{\mathbf{Q}}_{1} \hat{\mathbf{D}} \mathbf{A}_{3} \odot \mathbf{B}_{1}\right) \mathbf{A}_{4}\left(\mathbf{A}_{5} \hat{\mathbf{Q}}_{2} \mathbf{A}_{6} \odot \mathbf{B}_{2}\right) \phi\right]= \\
=\frac{1}{N} \mathbb{E} \operatorname{tr}\left[\mathbf{A}_{1}\left(\mathbf{A}_{2} \hat{\mathbf{Q}}_{1} \mathbf{D} \mathbf{A}_{3} \odot \mathbf{B}_{1}\right) \mathbf{A}_{4}\left(\mathbf{A}_{5} \hat{\mathbf{Q}}_{2} \mathbf{A}_{6} \odot \mathbf{B}_{2}\right) \phi\right] \\
+\mathcal{O}\left(N^{-1}\right) .
\end{array}
$$




$$
\begin{aligned}
& \begin{array}{r}
\sum_{r=1}^{M} \operatorname{tr}\left[\mathbf{A}_{1}\left(\mathbf{A}_{2} \mathbf{e}_{r} \mathbf{e}_{r}^{T} \mathbf{A}_{3} \odot \mathbf{B}_{1}\right) \mathbf{A}_{4}\left(\mathbf{A}_{5} \mathbf{e}_{r} \mathbf{e}_{r}^{T} \mathbf{A}_{6} \odot \mathbf{B}_{2}\right)\right] \\
=\operatorname{tr}\left[\left(\mathbf{A}_{3}^{T} \otimes \mathbf{A}_{2}\right) \Pi_{M}\left(\mathbf{A}_{5}^{T} \otimes \mathbf{A}_{6}\right) \operatorname{dgvec}\left(\mathbf{B}_{2}^{T}\right)\left(\mathbf{A}_{4}^{T} \otimes \mathbf{A}_{1}\right) \operatorname{dgvec}\left(\mathbf{B}_{1}\right)\right] \\
\sum_{r=1}^{M} \operatorname{tr}\left[\mathbf{A}_{1}\left(\mathbf{A}_{2} \mathbf{e}_{r} \mathbf{e}_{r}^{T} \mathbf{A}_{3} \odot \mathbf{B}_{1}\right) \mathbf{A}_{4}\left(\mathbf{A}_{5}\left(\mathbf{A}_{6} \mathbf{e}_{r} \mathbf{e}_{r}^{T} \mathbf{A}_{7} \odot \mathbf{B}_{2}\right) \mathbf{A}_{8} \odot \mathbf{B}_{3}\right)\right] \\
=\operatorname{tr}\left[\Pi_{M}\left(\mathbf{A}_{2}^{T} \otimes \mathbf{A}_{3}\right) \operatorname{dgvec}\left(\mathbf{B}_{1}^{T}\right)\left(\mathbf{A}_{1}^{T} \otimes \mathbf{A}_{4}\right) \operatorname{dgvec}\left(\mathbf{B}_{3}\right)\left(\mathbf{A}_{8}^{T} \otimes \mathbf{A}_{5}\right) \operatorname{dgvec}\left(\mathbf{B}_{2}\right)\left(\mathbf{A}_{7}^{T} \otimes \mathbf{A}_{6}\right)\right]
\end{array}
\end{aligned}
$$

$$
\begin{aligned}
& \sum_{i=1}^{M} \sum_{j=1}^{N} \operatorname{tr}\left[\mathbf{A}_{1}\left(\mathbf{A}_{2} \mathbf{e}_{i} \tilde{\mathbf{e}}_{j}^{T} \mathbf{A}_{3} \odot \mathbf{B}_{1}\right) \mathbf{A}_{4}\left(\mathbf{A}_{5} \mathbf{e}_{i} \tilde{\mathbf{e}}_{j}^{T} \mathbf{A}_{6} \odot \mathbf{B}_{2}\right)\right]= \\
&=\operatorname{tr}\left[\left(\mathbf{A}_{3}^{T} \otimes \mathbf{A}_{2}\right) \tilde{\Pi}_{M, N}\left(\mathbf{A}_{5}^{T} \otimes \mathbf{A}_{6}\right) \operatorname{dgvec}\left(\mathbf{B}_{2}^{T}\right)\left(\mathbf{A}_{4}^{T} \otimes \mathbf{A}_{1}\right) \operatorname{dgvec}\left(\mathbf{B}_{1}\right)\right]
\end{aligned}
$$

$$
\begin{aligned}
\sum_{i=1}^{M} \sum_{j=1}^{N} \operatorname{tr}\left[\mathbf{A}_{1}\left(\mathbf{A}_{2} \mathbf{e}_{i} \tilde{\mathbf{e}}_{j}^{T} \mathbf{A}_{3} \odot \mathbf{B}_{1}\right) \mathbf{A}_{4}\left(\mathbf{A}_{5}\left(\mathbf{A}_{6} \tilde{\mathbf{e}}_{j} \mathbf{e}_{i}^{T} \mathbf{A}_{7} \odot \mathbf{B}_{2}\right) \mathbf{A}_{8} \odot \mathbf{B}_{3}\right)\right] \\
=\operatorname{tr}\left[\left(\left(\mathbf{A}_{2} \mathbf{A}_{7}\right)^{T} \otimes \mathbf{A}_{6} \mathbf{A}_{3}\right) \operatorname{dgvec}\left(\mathbf{B}_{1}^{T}\right)\left(\mathbf{A}_{1}^{T} \otimes \mathbf{A}_{4}\right) \operatorname{dgvec}\left(\mathbf{B}_{3}\right)\left(\mathbf{A}_{8}^{T} \otimes \mathbf{A}_{5}\right) \operatorname{dgvec}\left(\mathbf{B}_{2}\right)\right]
\end{aligned}
$$

$$
\begin{aligned}
& \sum_{i=1}^{M} \sum_{j=1}^{N} \operatorname{tr}\left[\mathbf{A}_{1}\right.\left.\left(\mathbf{A}_{2} \mathbf{e}_{i} \tilde{\mathbf{e}}_{j}^{T} \mathbf{A}_{3} \odot \mathbf{B}_{1}\right) \mathbf{A}_{4}\left(\mathbf{A}_{5}\left(\mathbf{A}_{6} \mathbf{e}_{i} \tilde{\mathbf{e}}_{j}^{T} \mathbf{A}_{7} \odot \mathbf{B}_{2}\right) \mathbf{A}_{8} \odot \mathbf{B}_{3}\right)\right] \\
&=\operatorname{tr}\left[\tilde{\Pi}_{M, N}\left(\mathbf{A}_{2}^{T} \otimes \mathbf{A}_{3}\right) \operatorname{dgvec}\left(\mathbf{B}_{1}^{T}\right)\left(\mathbf{A}_{1}^{T} \otimes \mathbf{A}_{4}\right) \operatorname{dgvec}\left(\mathbf{B}_{3}\right)\left(\mathbf{A}_{8}^{T} \otimes \mathbf{A}_{5}\right) \operatorname{dgvec}\left(\mathbf{B}_{2}\right)\left(\mathbf{A}_{7}^{T} \otimes \mathbf{A}_{6}\right)\right]
\end{aligned}
$$

$$
\begin{aligned}
\mathbb{E}\left[\widetilde{\boldsymbol{F}}_{2}\left(z_{1}, z_{2}\right)\right]=\frac{z_{1}}{\omega_{1}} & \frac{1}{N} \mathbb{E} \operatorname{tr}\left[\mathbf{A}_{1}\left(\mathbf{A}_{2} \hat{\mathbf{Q}}_{1} \mathbf{R} \mathbf{A}_{3} \odot \mathbf{B}_{1}\right) \mathbf{A}_{4}\left(\mathbf{A}_{5} \hat{\mathbf{Q}}_{2} \mathbf{A}_{6} \odot \mathbf{B}_{2}\right) \phi\right] \\
& -\frac{z_{1}}{\omega_{1}} \frac{1}{N^{2}} \mathbb{E} \operatorname{tr}\left[\left(\mathbf{A}_{1} \odot\left(\mathbf{A}_{2} \hat{\mathbf{Q}}_{1} \mathbf{R} \hat{\mathbf{Q}}_{2} \mathbf{A}_{6}\right)^{T}\right) \mathbf{B}_{1}\left(\mathbf{A}_{4} \odot\left(\mathbf{A}_{5} \hat{\mathbf{Q}}_{2} \hat{\mathbf{R}} \mathbf{A}_{3}\right)^{T}\right) \mathbf{B}_{2} \phi\right] \\
& +\widetilde{\chi}_{1}\left(z_{1}, z_{2}\right)+\tilde{\chi}_{2}\left(z_{1}, z_{2}\right)+\mathcal{O}\left(N^{-\mathbb{N}}\right)
\end{aligned}
$$

$$
\begin{aligned}
& \tilde{\chi}_{1}\left(z_{1}, z_{2}\right)=\frac{z_{1}^{2} \frac{1+\varsigma}{\omega_{1}} \frac{\varsigma}{N^{2}} \mathbb{E} \operatorname{tr}}{}\left[\mathbf{A}_{1}\left(\mathbf{A}_{2} \hat{\mathbf{Q}}_{1} \operatorname{dg}\left(\hat{\mathbf{Q}}_{1} \mathbf{R}\right) \hat{\mathbf{R}} \mathbf{A}_{3} \odot \mathbf{B}_{1}\right) \mathbf{A}_{4}\left(\mathbf{A}_{5} \hat{\mathbf{Q}}_{2} \mathbf{A}_{6} \odot \mathbf{B}_{2}\right) \phi\right] \\
&-\frac{z_{1}}{\omega_{1}} \frac{\varsigma}{N^{2}} \mathbb{E} \operatorname{tr}\left[\mathbf{A}_{1}\left(\mathbf{A}_{2} \hat{\mathbf{Q}}_{1} \mathbf{R} \hat{\mathbf{Q}}_{1} \hat{\mathbf{R}} \mathbf{A}_{3} \odot \mathbf{B}_{1}\right) \mathbf{A}_{4}\left(\mathbf{A}_{5} \hat{\mathbf{Q}}_{2} \mathbf{A}_{6} \odot \mathbf{B}_{2}\right) \phi\right] \\
&+\frac{z_{1} z_{2}}{\omega_{1}} \frac{1+\varsigma}{N^{2}} \mathbb{E} \operatorname{tr}[\left.\left(\left(\hat{\mathbf{R}} \mathbf{A}_{3}\right)^{T} \otimes \mathbf{A}_{2} \hat{\mathbf{Q}}_{1} \mathbf{R}\right) \Pi_{M}\left(\left(\mathbf{A}_{5} \hat{\mathbf{Q}}_{2}\right)^{T} \otimes \hat{\mathbf{Q}}_{2} \mathbf{A}_{6}\right) \operatorname{dgvec}\left(\mathbf{B}_{2}^{T}\right)\left(\mathbf{A}_{4}^{T} \otimes \mathbf{A}_{1}\right) \operatorname{dgvec}\left(\mathbf{B}_{1}\right) \phi\right] \\
&-\frac{z_{1}}{\omega_{1}} \frac{\varsigma}{N^{2}} \mathbb{E} \operatorname{tr}\left[\tilde{\Pi}_{M}\left(\mathbf{A}_{2} \hat{\mathbf{Q}}_{1} \mathbf{R} \hat{\mathbf{Q}}_{2} \mathbf{A}_{5}^{T} \otimes \mathbf{A}_{3}^{T} \hat{\mathbf{R}} \hat{\mathbf{Q}}_{2} \mathbf{A}_{6}\right) \operatorname{dgvec}\left(\mathbf{B}_{2}^{T}\right)\left(\mathbf{A}_{4}^{T} \otimes \mathbf{A}_{1}\right) \operatorname{dgvec}\left(\mathbf{B}_{1}\right) \phi\right]
\end{aligned}
$$

Now, combining (155) and (153), replacing $\mathbf{A}_{3}$ by $\overline{\mathbf{Q}}_{1} \mathbf{A}_{3}$ and using Proposition 3 we obtain (95). Finally, using (95) in (153) we get to (96). This completes the proof of Proposition 4.

\section{Proof of Proposition 5}

We begin by proving the identity in (97). Replacing $\hat{\mathbf{R}}$ in the expression on the left hand side of (97) by its decomposition in (59), applying the the integration by parts formula in Proposition 2, and using Lemma 1, we obtain the result. The same apporach allows to prove the identity in (98). The fact 
that the variances of the two quantities decay as $\mathcal{O}\left(N^{-2}\right)$ is a direct consequence of the application of the PoincareNash inequality in Proposition 2, simply noticing that all the derivatives involved can be expressed in the form of the statement of Lemma 9.

\section{Proof of Proposition 6 (3 resolvents)}

The fact that $\operatorname{var}\left[\hat{\zeta}\left(\mathbf{A}_{1}, \mathbf{B}_{1}, \mathbf{B}_{2}\right)\right]=\mathcal{O}\left(N^{-2}\right)$ follows from the Poincaré-Nash inequality in (53) of Proposition 2. By taking derivatives of $\hat{\zeta}\left(\mathbf{A}_{1}, \mathbf{B}_{1}, \mathbf{B}_{2}\right)$ with respect to the entries $X_{i j}$ and $X_{i j}^{*}$ and applying Jensen's inequality we end up with a sum of terms that have the form in the statement of Lemma 9 above. Each of these terms can be written as $\mathcal{O}\left(N^{-2}\right)$, which directly shows that $\operatorname{var}\left[\hat{\zeta}\left(\mathbf{A}_{1}, \mathbf{B}_{1}, \mathbf{B}_{2}\right)\right]=\mathcal{O}\left(N^{-2}\right)$.

Next, let us analyze the expectation of $\hat{\zeta}\left(\mathbf{A}_{1}, \mathbf{B}_{1}, \mathbf{B}_{2}\right)$. By replacing the first appearance of the sample covariance matrix $\hat{\mathbf{R}}$ in $\hat{\zeta}\left(\mathbf{A}_{1}, \mathbf{B}_{1}, \mathbf{B}_{2}\right)$ with its decomposition (59) and applying the the integration by parts formula in Proposition 2, we are able to write

$$
\begin{aligned}
\mathbb{E}\left[\hat{\zeta}\left(\mathbf{A}_{1}, \mathbf{B}_{1}, \mathbf{B}_{2}\right)\right] \\
=\frac{1}{N} \mathbb{E} \operatorname{tr}\left[\mathbf{R}\left(\mathbf{A}_{1} \hat{\mathbf{Q}}_{1} \odot \mathbf{B}_{1}\right) \mathbf{R}\left(\hat{\mathbf{Q}}_{2} \hat{\mathbf{R}} \hat{\mathbf{Q}}_{2} \odot \mathbf{B}_{2}\right) \phi\right] \\
\quad-\frac{1}{N^{2}} \mathbb{E}\left[\operatorname{vdg}\left(\mathbf{A}_{1} \hat{\mathbf{Q}}_{1} \hat{\mathbf{R}}\right)^{T} \mathbf{B}_{1} \operatorname{vdg}\left(\mathbf{U}_{2} \hat{\mathbf{Q}}_{1}\right) \phi\right] \\
\quad-\frac{1}{N^{2}} \mathbb{E}\left[\operatorname{vdg}\left(\hat{\mathbf{Q}}_{2} \mathbf{U}_{1}\right)^{T} \mathbf{B}_{2} \operatorname{vdg}\left(\mathbf{R} \hat{\mathbf{Q}}_{2} \hat{\mathbf{R}} \hat{\mathbf{Q}}_{2}\right) \phi\right] \\
\quad-\frac{1}{N^{2}} \mathbb{E}\left[\operatorname{vdg}\left(\hat{\mathbf{Q}}_{2} \hat{\mathbf{R}} \hat{\mathbf{Q}}_{2} \mathbf{U}_{1}\right)^{T} \mathbf{B}_{2} \operatorname{vdg}\left(\mathbf{R} \hat{\mathbf{Q}}_{2}\right) \phi\right] \\
+\frac{1}{N^{2}} \mathbb{E}\left[\operatorname{vdg}\left(\hat{\mathbf{Q}}_{2} \mathbf{U}_{1}\right)^{T} \mathbf{B}_{2} \operatorname{vdg}\left(\mathbf{R} \hat{\mathbf{Q}}_{2}\right) \phi\right]+\mathcal{O}\left(N^{-1}\right)
\end{aligned}
$$

where $\operatorname{vdg}(\mathbf{A})$ is column vector with the diagonal entries of $\mathbf{A}$ and where the matrices $\mathbf{U}_{1}$ and $\mathbf{U}_{2}$ are defined as

$$
\begin{aligned}
& \mathbf{U}_{1}=\hat{\mathbf{R}}\left(\mathbf{A}_{1} \hat{\mathbf{Q}}_{1} \odot \mathbf{B}_{1}\right) \mathbf{R} \\
& \mathbf{U}_{2}=\mathbf{R}\left(\hat{\mathbf{Q}}_{2} \hat{\mathbf{R}} \hat{\mathbf{Q}}_{2} \odot \mathbf{B}_{2}\right) \mathbf{R} .
\end{aligned}
$$

Observing the form of the different terms in (156), we come to the conclusion that, apart from the first one, the rest can be expressed as $N^{-2} \mathbb{E}\left[\operatorname{vdg}\left(\mathbf{G}_{1}\right)^{T} \mathbf{B} \operatorname{vdg}\left(\mathbf{G}_{2}\right) \phi\right]$, where $\mathbf{G}_{1}$ and $\mathbf{G}_{2}$ are random square matrices with bounded spectral norm and where $\mathbf{B}$ is either $\mathbf{B}_{1}$ or $\mathbf{B}_{2}$. By definition, if $\mathbf{B}$ has also bounded spectral norm, one can establish that

$$
\begin{aligned}
& \frac{1}{N^{2}}\left|\operatorname{vdg}\left(\mathbf{G}_{1}\right)^{T} \mathbf{B} \operatorname{vdg}\left(\mathbf{G}_{2}\right) \phi\right| \leq \\
& \leq \frac{1}{N}\|\mathbf{B}\| \sqrt{\frac{\operatorname{tr}\left[\mathbf{G}_{1} \odot \mathbf{G}_{1}^{H}\right]}{N} \frac{\operatorname{tr}\left[\mathbf{G}_{2} \odot \mathbf{G}_{2}^{H}\right]}{N}}=\mathcal{O}\left(N^{-1}\right) .
\end{aligned}
$$

In this situation, we can disregard the corresponding terms in (156). Conversely, if we fix $\mathbf{B}=\mathbf{1 1}^{T}$ (an all-ones matrix), we will have

$$
\begin{aligned}
& \frac{1}{N^{2}} \mathbb{E}\left[\operatorname{vdg}\left(\mathbf{G}_{1}\right)^{T} \mathbf{B} \operatorname{vdg}\left(\mathbf{G}_{2}\right) \phi\right]=\mathbb{E}\left[\frac{1}{N} \operatorname{tr}\left(\mathbf{G}_{1}\right) \frac{1}{N} \operatorname{tr}\left(\mathbf{G}_{2}\right)\right] \\
= & \frac{1}{N} \mathbb{E} \operatorname{tr}\left(\mathbf{G}_{1}\right) \frac{1}{N} \mathbb{E} \operatorname{tr}\left(\mathbf{G}_{2}\right)+\mathbb{E}\left[\frac{1}{N} \operatorname{tr}\left(\mathbf{G}_{1}\right)\left(\frac{1}{N} \operatorname{tr}\left(\mathbf{G}_{2}\right)\right)^{\circ}\right] .
\end{aligned}
$$

In all these situations, it turns out that $N^{-1} \operatorname{tr}\left(\mathbf{G}_{1}\right)$ and $N^{-1} \operatorname{tr}\left(\mathbf{G}_{2}\right)$ are random quantities that can be described by Propositions 3 and 4 proven above. Therefore, in all these terms we can establish that $\mathbb{E}\left[N^{-2} \operatorname{tr}\left(\mathbf{G}_{1}\right)\left(\operatorname{tr}\left(\mathbf{G}_{2}\right)\right)^{\circ}\right]=$ $\mathcal{O}\left(N^{-1}\right)$, and we can provide a deterministic equivalent for both $N^{-1} \mathbb{E} \operatorname{tr}\left(\mathbf{G}_{1}\right)$ and $N^{-1} \mathbb{E} \operatorname{tr}\left(\mathbf{G}_{2}\right)$ up to an error of order $\mathcal{O}\left(N^{-1}\right)$. In conclusion, we can easily establish the behavior all the terms in (156) of the form $N^{-2} \mathbb{E}\left[\operatorname{vdg}\left(\mathbf{G}_{1}\right)^{T} \operatorname{B} \operatorname{vdg}\left(\mathbf{G}_{2}\right) \phi\right]$.

Regarding the first term on the right hand of (156), it will be shown below that it can be expressed as in (157) at the top of the next page. By Lemma 1, the first term on the right hand side of (157) is always of order one. The next three terms can be asymptotically neglected if at least one of the two matrices $\mathbf{B}_{1}$ or $\mathbf{B}_{2}$ have bounded spectral norm. Indeed, observe that all these terms can be written as $N^{-2} \mathbb{E} \operatorname{tr}\left[\mathbf{B}_{1} \mathbf{G}_{1} \mathbf{B}_{2} \mathbf{G}_{2} \phi\right]$ for some random square matrices $\mathbf{G}_{1}, \mathbf{G}_{2}$ that have bounded spectral norm. Since both $\mathbf{B}_{1}$ and $\mathbf{B}_{2}$ are Hermitian positive definite, it suffices that one of them (e.g. $\mathbf{B}_{1}$ ) has bounded spectral norm to be able to write

$$
\begin{aligned}
\left|\frac{1}{N^{2}} \operatorname{tr}\left[\mathbf{B}_{1} \mathbf{G}_{1} \mathbf{B}_{2} \mathbf{G}_{2} \phi\right]\right| & \leq\left\|\mathbf{B}_{1} \mathbf{G}_{1} \mathbf{G}_{2} \phi\right\| \frac{1}{N^{2}} \operatorname{tr}\left[\mathbf{B}_{2}\right] \\
& =\mathcal{O}\left(N^{-1}\right) .
\end{aligned}
$$

This means that, for the purposes of the proof of Proposition 6 , we only need to consider the last terms in (157) in the case where $\mathbf{B}_{1}=\mathbf{B}_{2}=\mathbf{1 1}^{T}$.

We have now all the ingredients to finalize the proof of Proposition 6. Assume first that both $\mathbf{B}_{1}$ and $\mathbf{B}_{2}$ have bounded spectral norm. In this case, only the first terms on the right hand side of (156) and (157) are of order one, which directly proves (99). If $\mathbf{B}_{1}=\mathbf{1 1}^{T}$ and $\mathbf{B}_{2}$ has bounded spectral norm, we should also consider the first term in (157) and the first two terms in (156). Inserting the first term of (157) into (156) and applying Proposition 3 and (157) with $\mathbf{A}_{1}=\mathbf{I}_{M}$ we directly obtain (100). Assume next that $\mathbf{B}_{2}=\mathbf{1 1}^{T}$ and $\mathbf{B}_{1}$ having bounded spectral norm. In this case, we only need to consider the first term in (157), together with the 1st, 3rd, 4th and 5th terms on the right hand side of (156). We can deal with the non-vanishing terms of (156) as follows. For the first term, we directly use (157). The third and fifth terms can be described using Propositions 3 and 4. Regarding the fourth term, using Proposition 3 we are able to write it as $-\mathbb{E}\left[\hat{\zeta}\left(\mathbf{A}_{1}, \mathbf{B}_{1}, \mathbf{B}_{2}\right)\right] N^{-1} \operatorname{tr}\left(\mathbf{R} \overline{\mathbf{Q}}_{2}\right)$ plus some error $\mathcal{O}\left(N^{-1}\right)$, where we notice that $\mathbb{E}\left[\hat{\zeta}\left(\mathbf{A}_{1}, \mathbf{B}_{1}, \mathbf{B}_{2}\right)\right]$ is precisely the quantity that we are trying to evaluate, i.e. the left hand side of (156). Moving this fourth term to the other side of the equality and applying the identity in (69) we directly obtain (101). Consider finally the case $\mathbf{B}_{1}=\mathbf{B}_{2}=\mathbf{1 1}^{T}$. In this situation, all the terms on the right hand side of both (157) 


$$
\begin{aligned}
& \frac{1}{N} \mathbb{E} \operatorname{tr}\left[\mathbf{R}\left(\mathbf{A}_{1} \hat{\mathbf{Q}}_{1} \odot \mathbf{B}_{1}\right) \mathbf{R}\left(\hat{\mathbf{Q}}_{2} \hat{\mathbf{R}} \hat{\mathbf{Q}}_{2} \odot \mathbf{B}_{2}\right)\right]=\left(\frac{z_{2}}{\omega_{2}}\right)^{2} \frac{1}{1-\Gamma_{22}} \frac{1}{N} \mathbb{E} \operatorname{tr}\left[\mathbf{R}\left(\mathbf{A}_{1} \overline{\mathbf{Q}}_{1} \odot \mathbf{B}_{1}\right) \mathbf{R}\left(\overline{\mathbf{Q}}_{2} \mathbf{R} \overline{\mathbf{Q}}_{2} \odot \mathbf{B}_{2}\right)\right] \\
&+\frac{z_{1}}{\omega_{1}}\left(\frac{z_{2}}{\omega_{2}}\right)^{2} \frac{1}{1-\Gamma_{22}} \frac{1}{N^{2}} \mathbb{E} \operatorname{tr}\left[\left(\mathbf{R} \odot\left(\mathbf{A}_{1} \hat{\mathbf{Q}}_{1} \mathbf{R} \hat{\mathbf{Q}}_{2}\right)^{T}\right) \mathbf{B}_{1}\left(\mathbf{R} \odot\left(\overline{\mathbf{Q}}_{2} \mathbf{R} \hat{\mathbf{Q}}_{2} \hat{\mathbf{R}} \overline{\mathbf{Q}}_{1}\right)^{T}\right) \mathbf{B}_{2}\right] \\
&+\left(\frac{z_{2}}{\omega_{2}}\right)^{3} \frac{1}{1-\Gamma_{22}} \frac{1}{N^{2}} \operatorname{tr}\left[\left(\mathbf{R} \odot\left(\mathbf{A}_{1} \hat{\mathbf{Q}}_{1} \hat{\mathbf{R}} \overline{\mathbf{Q}}_{2} \mathbf{R} \hat{\mathbf{Q}}_{2}\right)^{T}\right) \mathbf{B}_{1}\left(\mathbf{R} \odot\left(\hat{\mathbf{Q}}_{2} \mathbf{R} \hat{\mathbf{Q}}_{1}\right)^{T}\right) \mathbf{B}_{2}\right] \\
& \quad-\frac{z_{2}}{\omega_{2}} \frac{1}{N^{2}} \operatorname{tr}\left[\left(\mathbf{R} \odot\left(\mathbf{A}_{1} \hat{\mathbf{Q}}_{1} \hat{\mathbf{R}} \hat{\mathbf{Q}}_{2}\right)^{T}\right) \mathbf{B}_{1}\left(\mathbf{R} \odot\left(\hat{\mathbf{Q}}_{2} \mathbf{R} \hat{\mathbf{Q}}_{1}\right)^{T}\right) \mathbf{B}_{2}\right]+\mathcal{O}\left(N^{-1}\right) .
\end{aligned}
$$

and (156) need to be considered. The right hand side of (157) can be fully characterized using the third identity in Lemma 8 together with Proposition 4 . The result can be directly used to replace the first term on the right hand side of (156). The second term can be handled using Proposition 3 together with the identity in (157) particularized to the case $\mathbf{A}_{1}=\mathbf{I}_{M}$. As for the other terms of (156), they can be expanded as in the previous case, which leads directly to (102). This concludes the proof of this proposition.

\section{E. Proof of (157)}

Consider the two random functions $\digamma_{3}=\digamma_{3}\left(z_{1}, z_{2}\right)$ and $\widetilde{\digamma}_{3}=\widetilde{\digamma}_{3}\left(z_{1}, z_{2}\right)$ defined as

$$
\begin{aligned}
& \digamma_{3}=\frac{1}{N} \operatorname{tr}\left[\mathbf{R}\left(\mathbf{A}_{1} \hat{\mathbf{Q}}_{1} \odot \mathbf{B}_{1}\right) \mathbf{R}\left(\hat{\mathbf{Q}}_{2} \mathbf{A}_{2} \hat{\mathbf{Q}}_{2} \odot \mathbf{B}_{2}\right)\right] \\
& \widetilde{\digamma}_{3}=\frac{1}{N} \operatorname{tr}\left[\mathbf{R}\left(\mathbf{A}_{1} \hat{\mathbf{Q}}_{1} \odot \mathbf{B}_{1}\right) \mathbf{R}\left(\hat{\mathbf{Q}}_{2} \hat{\mathbf{R}} \mathbf{A}_{2} \hat{\mathbf{Q}}_{2} \odot \mathbf{B}_{2}\right)\right] .
\end{aligned}
$$

where $\mathbf{A}_{1}, \mathbf{A}_{2}$ are assumed to have bounded spectral norm, whereas $\mathbf{B}_{1}, \mathbf{B}_{2}$ are Hermitian positive semidefinite with bounded entries. Observe that we can recover (157) by fixing $\mathbf{A}_{2}=\mathbf{I}_{M}$ in $\mathbb{E} \widetilde{\digamma}_{3}$.

Consider first the expectation $\mathbb{E}\left[\widetilde{\digamma}_{3}\left(z_{1}, z_{2}\right)\right]$. Replacing $\hat{\mathbf{R}}$ in the definition of $\widetilde{\digamma}_{3}\left(z_{1}, z_{2}\right)$ by its decomposition in (59) and using the integration by parts formula in Proposition 2 together with Lemmas 10 and 11 and Proposition 4, we are able to write the expression in (158) at the top of the next page. Using the same techniques, one can also show that

$$
\begin{array}{r}
\frac{1}{N} \mathbb{E} \operatorname{tr}\left[\mathbf{R}\left(\mathbf{A}_{1} \hat{\mathbf{Q}}_{1} \odot \mathbf{B}_{1}\right) \mathbf{R}\left(\hat{\mathbf{Q}}_{2} \hat{\mathbf{D}} \mathbf{A}_{2} \hat{\mathbf{Q}}_{2} \odot \mathbf{B}_{2}\right)\right]= \\
=\frac{1}{N} \mathbb{E} \operatorname{tr}\left[\mathbf{R}\left(\mathbf{A}_{1} \hat{\mathbf{Q}}_{1} \odot \mathbf{B}_{1}\right) \mathbf{R}\left(\hat{\mathbf{Q}}_{2} \mathbf{D} \mathbf{A}_{2} \hat{\mathbf{Q}}_{2} \odot \mathbf{B}_{2}\right)\right] \\
+\mathcal{O}\left(N^{-1}\right) .
\end{array}
$$

Combining this last equation with (158) and replacing $\mathbf{A}_{2}$ with $\overline{\mathbf{Q}}_{2} \mathbf{A}_{2}$ we obtain (159) on the next page. Observe that the third term of the right hand side contains a slightly modified version of $\digamma_{3}\left(z_{1}, z_{2}\right)$ in which $\mathbf{A}_{2}$ has been replaced by $\mathbf{R}$. Hence, particularizing the above expression for $\mathbf{A}_{2}=\mathbf{R}$ and expanding the first two terms on the right hand side with Proposition 4, we obtain (160) on the next page. Using this in (158) after setting $\mathbf{A}_{2}$ equal to the identity matrix, we obtain (157).

\section{APPENDIX E \\ PROOF OF PROPOSITION 2}

Using the same approach as in [39, Lemma 3.9], one can see that $\phi_{M}$ is a $\mathcal{C}^{1}$ function in $\mathbb{R}^{2 M N}\left(\mathbb{R}^{M N}\right.$ for real-valued entries) with partial derivatives given by

$$
\begin{aligned}
& \frac{\partial \phi_{M}}{\partial X_{i j}^{*}}=\frac{\partial}{\partial X_{i j}^{*}} \operatorname{det}\left(\phi\left(\hat{\mathbf{R}}_{M}\right)\right)= \\
& =\frac{1+\varsigma}{N} \mathbf{e}_{i}^{T} \mathbf{R}_{M}^{1 / 2} \operatorname{adj}\left(\phi\left(\hat{\mathbf{R}}_{M}\right)\right) \phi^{\prime}\left(\hat{\mathbf{R}}_{M}\right) \mathbf{R}_{M}^{1 / 2} \mathbf{x}_{j}
\end{aligned}
$$

where $\operatorname{adj}(\mathbf{A})$ is the adjugate of $\mathbf{A}$. Given the fact that $\phi$ and $\phi^{\prime}$ are bounded, we see that the partial derivatives of $\phi_{M}$ are polynomically bounded, because

$$
\begin{aligned}
& \sum_{i=1}^{M} \sum_{j=1}^{N} \mathbb{E}\left[\left|\frac{\partial \phi_{M}}{\partial X_{i j}^{*}}\right|^{2}\right] \\
& =\frac{(1+\varsigma)^{2}}{N} \mathbb{E} \operatorname{tr}\left[\mathbf{R}_{M}\left(\operatorname{adj}\left(\phi\left(\hat{\mathbf{R}}_{M}\right)\right) \phi^{\prime}\left(\hat{\mathbf{R}}_{M}\right)\right)^{2} \hat{\mathbf{R}}_{M}\right] \\
& \leq\left\|\mathbf{R}_{M}\right\| \frac{(1+\varsigma)^{2}}{N} \mathbb{E}\left[\sum_{m=1}^{M} \hat{\lambda}_{m}\left(\phi^{\prime}\left(\hat{\lambda}_{m}\right)\right)^{2} \prod_{\substack{j=1 \\
j \neq m}}^{M} \phi^{2}\left(\hat{\lambda}_{j}\right)\right] .
\end{aligned}
$$

Noting that $\phi^{\prime}$ is zero outside the blow-up $\mathcal{S}_{2 \epsilon}$ defined in Section III, we reach the conclusion that the right hand side of the above equation is clearly bounded, and therefore so are the partial derivatives of $\phi_{M}$.

Let us now establish the identity in (54). The identity is trivial for $r=1$, so we can assume $r>1$. Observe that we can write

$$
\begin{aligned}
\mathbb{E}\left[\Omega\left(\mathbf{X}, \mathbf{X}^{*}, z\right) \phi_{M}^{r}\right] & =\mathbb{E}\left[\Omega\left(\mathbf{X}, \mathbf{X}^{*}, z\right) \phi_{M}\right] \\
& +\mathbb{E}\left[\Omega\left(\mathbf{X}, \mathbf{X}^{*}, z\right) \phi_{M}\left(\phi_{M}^{r-1}-1\right)\right]
\end{aligned}
$$

so that, by the Cauchy-Schwarz inequality,

$$
\begin{aligned}
& \left|\mathbb{E}\left[\Omega\left(\mathbf{X}, \mathbf{X}^{*}, z\right) \phi_{M}\left(\phi_{M}^{r-1}-1\right)\right]\right|^{2} \\
& \leq \mathbb{E}\left[\left|\Omega\left(\mathbf{X}, \mathbf{X}^{*}, z\right) \phi_{M}\right|^{2}\right] \mathbb{E}\left[\left(1-\phi_{M}^{r-1}\right)^{2}\right] .
\end{aligned}
$$

Hence, it is sufficient to see that $\mathbb{E}\left[\left(1-\phi_{M}^{r}\right)^{2}\right]=\mathcal{O}\left(N^{-\ell}\right)$ for every $\ell \in \mathbb{N}$ and $r>1$. Let $\mathcal{E}$ denote the event that at least one non-zero eigenvalue of the sample covariance matrix $\hat{\lambda}_{j}$ is outside the support $\mathcal{S}$. Now, from the definition of $\phi$ we have $\phi_{M} \geq \mathbb{I}\left\{\mathcal{E}^{c}\right\}$, where $\mathbb{I}\{\cdot\}$ is the indicator function. This 


$$
\begin{aligned}
\mathbb{E}\left[\widetilde{\digamma}_{3}\left(z_{1}, z_{2}\right)\right]=\frac{z_{2}}{\omega_{2}} \frac{1}{N} \operatorname{tr} & {\left[\mathbf{R}\left(\mathbf{A}_{1} \hat{\mathbf{Q}}_{1} \odot \mathbf{B}_{1}\right) \mathbf{R}\left(\hat{\mathbf{Q}}_{2} \mathbf{R} \mathbf{A}_{2} \hat{\mathbf{Q}}_{2} \odot \mathbf{B}_{2}\right)\right] } \\
& -\frac{z_{2}}{\omega_{2}} \frac{1}{N^{2}} \operatorname{tr}\left[\left(\mathbf{R} \odot\left(\hat{\mathbf{Q}}_{2} \mathbf{R} \hat{\mathbf{Q}}_{1}\right)^{T}\right) \mathbf{B}_{2}\left(\mathbf{R} \odot\left(\mathbf{A}_{1} \hat{\mathbf{Q}}_{1} \hat{\mathbf{R}} \mathbf{A}_{2} \hat{\mathbf{Q}}_{2}\right)^{T}\right) \mathbf{B}_{1}\right] \\
& -\left(\frac{z_{2}}{\omega_{2}}\right)^{2} \frac{1}{N} \mathbb{E} \operatorname{tr}\left[\mathbf{R}\left(\mathbf{A}_{1} \hat{\mathbf{Q}}_{1} \odot \mathbf{B}_{1}\right) \mathbf{R}\left(\hat{\mathbf{Q}}_{2} \mathbf{R} \hat{\mathbf{Q}}_{2} \odot \mathbf{B}_{2}\right)\right] \frac{1}{N} \operatorname{tr}\left[\mathbf{A}_{2} \overline{\mathbf{Q}}_{2} \mathbf{R}\right]+\mathcal{O}\left(N^{-1}\right)
\end{aligned}
$$

$$
\begin{aligned}
\mathbb{E}\left[\digamma_{3}\left(z_{1}, z_{2}\right)\right]=\frac{1}{N} \mathbb{E} \operatorname{tr} & {\left[\mathbf{R}\left(\mathbf{A}_{1} \hat{\mathbf{Q}}_{1} \odot \mathbf{B}_{1}\right) \mathbf{R}\left(\overline{\mathbf{Q}}_{2} \mathbf{A}_{2} \hat{\mathbf{Q}}_{2} \odot \mathbf{B}_{2}\right)\right] } \\
& +\frac{z_{2}}{\omega_{2}} \frac{1}{N^{2}} \operatorname{tr}\left[\left(\mathbf{R} \odot\left(\hat{\mathbf{Q}}_{2} \mathbf{R} \hat{\mathbf{Q}}_{1}\right)^{T}\right) \mathbf{B}_{2}\left(\mathbf{R} \odot\left(\mathbf{A}_{1} \hat{\mathbf{Q}}_{1} \hat{\mathbf{R}} \overline{\mathbf{Q}}_{2} \mathbf{A}_{2} \hat{\mathbf{Q}}_{2}\right)^{T}\right) \mathbf{B}_{1}\right] \\
& +\left(\frac{z_{2}}{\omega_{2}}\right)^{2} \frac{1}{N} \mathbb{E} \operatorname{tr}\left[\mathbf{R}\left(\mathbf{A}_{1} \hat{\mathbf{Q}}_{1} \odot \mathbf{B}_{1}\right) \mathbf{R}\left(\hat{\mathbf{Q}}_{2} \mathbf{R} \hat{\mathbf{Q}}_{2} \odot \mathbf{B}_{2}\right)\right] \frac{1}{N} \mathbb{E} \operatorname{tr}\left[\overline{\mathbf{Q}}_{2} \mathbf{A}_{2} \overline{\mathbf{Q}}_{2} \mathbf{R}\right]+\mathcal{O}\left(N^{-1}\right)
\end{aligned}
$$

$$
\begin{aligned}
\mathbb{E}\left[\digamma_{3}\left(z_{1}, z_{2}\right)\right]_{\mathbf{A}_{2}=\mathbf{R}}= & \frac{1}{1-\Gamma_{22}} \frac{1}{N} \mathbb{E} \operatorname{tr}\left[\mathbf{R}\left(\mathbf{A}_{1} \overline{\mathbf{Q}}_{1} \odot \mathbf{B}_{1}\right) \mathbf{R}\left(\overline{\mathbf{Q}}_{2} \mathbf{R} \overline{\mathbf{Q}}_{2} \odot \mathbf{B}_{2}\right)\right] \\
+ & \frac{z_{1} / \omega_{1}}{1-\Gamma_{22}} \frac{1}{N^{2}} \mathbb{E} \operatorname{tr}\left[\left(\mathbf{R} \odot\left(\mathbf{A}_{1} \hat{\mathbf{Q}}_{1} \mathbf{R} \hat{\mathbf{Q}}_{2}\right)^{T}\right) \mathbf{B}_{1}\left(\mathbf{R} \odot\left(\overline{\mathbf{Q}}_{2} \mathbf{R} \hat{\mathbf{Q}}_{2} \hat{\mathbf{R}} \overline{\mathbf{Q}}_{1}\right)^{T}\right) \mathbf{B}_{2}\right] \\
& \quad+\frac{z_{2} / \omega_{2}}{1-\Gamma_{22}} \frac{1}{N^{2}} \operatorname{tr}\left[\left(\mathbf{R} \odot\left(\mathbf{A}_{1} \hat{\mathbf{Q}}_{1} \hat{\mathbf{R}} \overline{\mathbf{Q}}_{2} \mathbf{R} \hat{\mathbf{Q}}_{2}\right)^{T}\right) \mathbf{B}_{1}\left(\mathbf{R} \odot\left(\hat{\mathbf{Q}}_{2} \mathbf{R} \hat{\mathbf{Q}}_{1}\right)^{T}\right) \mathbf{B}_{2}\right]+\mathcal{O}\left(N^{-1}\right)
\end{aligned}
$$

implies that $\phi_{M}^{r} \geq \mathbb{I}\left(\mathcal{E}^{c}\right)$ and $\left(1-\phi_{M}^{r}\right)^{2} \leq \mathbb{I}(\mathcal{E})$. Taking expectations, we see that $\mathbb{E}\left[\left(1-\phi_{M}^{r-1}\right)^{2}\right] \leq \mathbb{P}(\mathcal{E})$. However, following [39, Proposition 3.1] we can establish that $\mathbb{P}(\mathcal{E})=$ $\mathcal{O}\left(N^{-\ell}\right)$ for every $\ell \in \mathbb{N}$, and this proves the result.

It remains to establish the last identity in Proposition 2. Using the expression of the partial derivatives of $\phi_{M}$ given in (161), together with the Cauchy-Schwarz inequality, we see that

$$
\begin{aligned}
& \sum_{i=1}^{M} \sum_{j=1}^{N}\left|\mathbb{E}\left[\Omega\left(\mathbf{X}, \mathbf{X}^{*}, z\right) \frac{\partial \phi_{M}}{\partial X_{i j}^{*}}\right]\right|^{2} \leq \mathbb{E}\left[\left|\Omega\left(\mathbf{X}, \mathbf{X}^{*}, z\right)\right|^{2}\right] \times \\
& \times \frac{(1+\varsigma)^{2}}{N} \mathbb{E} \operatorname{tr}\left[\mathbf{R}_{M}\left(\operatorname{adj}\left(\phi\left(\hat{\mathbf{R}}_{M}\right)\right) \phi^{\prime}\left(\hat{\mathbf{R}}_{M}\right)\right)^{2} \hat{\mathbf{R}}_{M}\right]
\end{aligned}
$$

However, noting that $\phi^{\prime}\left(\hat{\lambda}_{m}\right) \leq K \mathbb{I}(\mathcal{E})$, where $\mathcal{E}$ is the event that at least one eigenvalue is outside $\hat{\lambda}_{m}$ the support and where $K$ is an upper bound on $\phi^{\prime}$. Hence,

$$
\begin{aligned}
\frac{1}{N} \mathbb{E} \operatorname{tr}\left[\mathbf{R}_{M}\left(\operatorname{adj}\left(\phi\left(\hat{\mathbf{R}}_{M}\right)\right) \phi^{\prime}\left(\hat{\mathbf{R}}_{M}\right)\right)^{2} \hat{\mathbf{R}}_{M}\right] \leq \\
\times\left\|\mathbf{R}_{M}\right\| \frac{1}{N} \mathbb{E}\left[\sum_{m=1}^{M} \hat{\lambda}_{m}\left(\phi^{\prime}\left(\hat{\lambda}_{m}\right)\right)^{2} \times\right. \\
\left.\times \prod_{\substack{j=1 \\
j \neq m}}^{M} \phi^{2}\left(\hat{\lambda}_{j}\right)\right] \leq K\left\|\mathbf{R}_{M}\right\| \mathbb{P}(\mathcal{E})
\end{aligned}
$$

for some positive constant $K$ independent of $M$, where we used the fact that $\hat{\lambda}_{m} \phi^{\prime}\left(\hat{\lambda}_{m}\right)$ is zero for $\hat{\lambda}_{m}$ outside $\mathcal{S}_{2 \epsilon}$ and the fact that $\phi$ is bounded. Using the reasoning above, we see that $\mathbb{P}(\mathcal{E})=\mathcal{O}\left(N^{-\mathbb{N}}\right)$, which concludes the proof.

\section{APPENDIX F}

Bounds ON $\omega_{M}(z)$ AND $\Gamma_{M}\left(\omega_{M}\left(z_{1}\right), \omega_{M}\left(z_{2}\right)\right)$

Lemma 12: Under (As1) - (As3), and for any $z \in$ $\mathbb{C} \backslash(\mathcal{T} \cup\{0\}), \mathcal{T}$ being defined in Lemma $2,\left|\omega_{M}(z)\right|$ is bounded above and away from zero for all $M$. Furthermore, these bounds hold uniformly for all $z$ on $\mathcal{C}$.

Proof: Assume that there exists a subsequence $\left(M_{n}\right)$ for which $\left|\omega_{M_{n}}(z)\right| \rightarrow \infty$. Using (15) we readily see that this would imply

$$
\left|1-\frac{1}{N} \operatorname{tr}\left[\mathbf{R}_{M_{n}}\left(\mathbf{R}_{M_{n}}-\omega_{M_{n}}(z) \mathbf{D}_{M_{n}}\right)^{-1}\right]\right| \rightarrow 0
$$

However, by Jensen's inequality

$$
\begin{aligned}
\mid \frac{1}{N} \operatorname{tr}\left[\mathbf { R } _ { M _ { n } } \left(\mathbf{R}_{M_{n}}\right.\right. & \left.\left.-\omega_{M_{n}}(z) \mathbf{D}_{M_{n}}\right)^{-1}\right]\left.\right|^{2} \\
& \leq \frac{1}{N} \sum_{m=1}^{\bar{M}} K_{m} \frac{\gamma_{m}^{2}}{\left|\gamma_{m}-\omega_{M_{n}}(z)\right|^{2}} \rightarrow 0
\end{aligned}
$$

which contradicts (162). Next, assume that there exists a subsequence $\left(M_{n}\right)$ for which $\left|\omega_{M_{n}}(z)\right| \rightarrow 0$. From (15) we readily see that this would imply that

$$
\left|\frac{1}{N} \operatorname{tr}\left[\mathbf{R}_{M_{n}}\left(\mathbf{R}_{M_{n}}-\omega_{M_{n}}(z) \mathbf{D}_{M_{n}}\right)^{-1}\right]\right| \rightarrow \infty .
$$

However, since $\omega_{M_{n}}(z) \rightarrow 0$ the above quantity will be asymptotically upper bounded by $\lim \sup c_{M}<\infty$, leading to 
a contradiction. The above reasoning is valid if $z$ is replaced by a sequence of points $z_{n}$ on $\mathcal{C}$, implying that the bound holds uniformly on $\mathcal{C}$.

Lemma 13: Under (As1) - (As3), for any $z_{1}, z_{2} \in$ $\mathbb{C} \backslash(\mathcal{T} \cup\{0\}), \mathcal{T}$ being defined in Lemma 2, we have

$$
\sup _{M}\left|\Gamma_{M}\left(\omega_{M}\left(z_{1}\right), \omega_{M}\left(z_{2}\right)\right)\right|<1
$$

Furthermore, this bound holds uniformly over $\mathcal{C} \times \mathcal{C}$.

Proof: A direct application of the Cauchy-Schwarz inequality leads to

$$
\begin{aligned}
\left|\Gamma_{M}\left(\omega_{M}\left(z_{1}\right), \omega_{M}\left(z_{2}\right)\right)\right|^{2} & \leq \frac{1}{N} \sum_{m=1}^{\bar{M}} K_{m} \frac{\gamma_{m}^{2}}{\left|\gamma_{m}-\omega_{M}\left(z_{1}\right)\right|^{2}} \times \\
& \times \frac{1}{N} \sum_{m=1}^{\bar{M}} K_{m} \frac{\gamma_{m}^{2}}{\left|\gamma_{m}-\omega_{M}\left(z_{2}\right)\right|^{2}}
\end{aligned}
$$

so it is sufficient to prove that

$$
\sup _{z \in \mathcal{C}} \sup _{M} \frac{1}{N} \sum_{m=1}^{\bar{M}} K_{m} \frac{\gamma_{m}^{2}}{\left|\gamma_{m}-\omega_{M}(z)\right|^{2}}<1 .
$$

We first point out that it is known [35] that the above upper bound is valid for each fixed $M$ and $z \notin \mathcal{T} \cup\{0\}$. so we only need to show that it holds uniformly in these two parameters. First, it is shown in [35] that, for each $M$, there exists a $x_{M} \in$ $\mathcal{T}$ (which may depend on $M$ ) such that

$$
1=\frac{1}{N} \sum_{m=1}^{\bar{M}} K_{m} \frac{\gamma_{m}^{2}}{\left|\gamma_{m}-\omega_{M}\left(x_{M}\right)\right|^{2}} .
$$

By definition, we have

$$
\begin{aligned}
& x-z=\left(\omega_{M}\left(x_{M}\right)-\omega_{M}(z)\right) \times \\
& \times\left(1-\frac{1}{N} \sum_{m=1}^{\bar{M}} K_{m} \frac{\gamma_{m}^{2}}{\left(\gamma_{m}-\omega_{M}\left(x_{M}\right)\right)\left(\gamma_{m}-\omega_{M}(z)\right)}\right) .
\end{aligned}
$$

Now, since $x$ belongs to $\mathcal{T}$ (which does not depend on $M$ ) and since $\mathcal{C}$ encloses $\mathcal{T}$ and not zero, we see that $\inf _{M} \inf _{z \in \mathcal{C}}\left|x_{M}-z\right|>0$, so that, in particular $\inf _{M} \inf _{z \in \mathcal{C}}\left|\omega_{M}\left(x_{M}\right)-\omega_{M}(z)\right|>0$ and

$\inf _{M} \inf _{z \in \mathcal{C}}\left|1-\frac{1}{N} \sum_{m=1}^{\bar{M}} K_{m} \frac{\gamma_{m}^{2}}{\left(\gamma_{m}-\omega_{M}\left(x_{M}\right)\right)\left(\gamma_{m}-\omega_{M}(z)\right)}\right|>0$.

Using Cauchy-Schwarz and (164) we see that

$$
\begin{aligned}
\left|\frac{1}{N} \sum_{m=1}^{\bar{M}} K_{m} \frac{\gamma_{m}^{2}}{\left(\gamma_{m}-\omega_{M}\left(x_{M}\right)\right)\left(\gamma_{m}-\omega_{M}(z)\right)}\right|^{2} \\
\leq \frac{1}{N} \sum_{m=1}^{\bar{M}} K_{m} \frac{\gamma_{m}^{2}}{\left|\gamma_{m}-\omega_{M}(z)\right|^{2}} \leq 1
\end{aligned}
$$

for each $M$. However, (165) implies that the above inequality is strict, and holds uniformly in $M$ and $z \in \mathcal{C}$, as we wanted to prove.

\section{REFERENCES}

[1] A. Leshem and A. van der Veen, "Multichannel detection of gaussian signals with uncalibrated receivers," IEEE Sign, vol. 8, pp. 120-122, Apr. 2001.

[2] A. Leshem and A. V. der Veen, "Multichannel detection and spatial signature estimation with uncalibrated receivers," in Proceedings of the IEEE Workshop on Statistical Signal Processing, pp. 190-193, Aug 2001.

[3] T. Jiang, "The limiting distributions of eigenvalues of sample correlation matrices," Sankhya: The Indian Journal of Statistics, vol. 66, pp. 35-48, Feb. 2004.

[4] I. Johnstone, "On the distribution of the largest eigenvalue in principal component analysis," The Annals of Statistics, vol. 29, no. 2, pp. 295$327,2001$.

[5] J. Schott, "Testing for complete independence in high dimensions," Biometrika, vol. 92, pp. 951-956, Dec. 2005.

[6] T. Anderson, An Introduction to Multivariate Statistical Analysis. Stanford: John Wiley and Sons, 1984.

[7] H. Hotelling, "New light on the correlation coefficient and its transforms," Journal of the Royal Statistical Society, Series B (Methodological), vol. 15, no. 2, pp. 193-232, 1953.

[8] Z. Bao, G. Pan, and W. Zhou, "Tracy-Widom law for the extreme eigenvalues of sample correlation matrices," Electronic Journal of Statistics, vol. 17 , no. 88 , pp. 1-32, 2012.

[9] N. Pillai and J. Lin, "Edge universality if correlation matrices," The Annals of Statistics, vol. 40, no. 3, pp. 1737-1763, 2012.

[10] T. Jiang, "The asymptotic distributions of the largest entries of sample correlation matrices," The Annals of Applied Probability, vol. 14, no. 2, pp. 865-880, 2004.

[11] W. Zhou, "Asymptotic distribution of the largest off-diagonal entry of correlation matrices," Transactions of the American Mathematical Society, vol. 359, pp. 5345-5363, Nov. 2007.

[12] T. Cai and T. Jiang, "Phase transition in limiting distributions of coherence of high-dimensionamatrices matrices," Journal of Multivariate Analysis, vol. 107, pp. 24-39, 2012

[13] T. Cai and T. Jiang, "Limiting laws of coherence of random matrices with applications to testing covariance structure and construction of compressed sensing matrices," Annals of Stati, vol. 39, pp. 1496-1525, 2011.

[14] B. Shao and W. Zhou, "Necessary and sufficient conditions for the asymptotic distributions of coherence of ultra-high dimensional random matrices," The Annals of Probability, vol. 42, no. 2, pp. 623-648, 2014.

[15] D. Ramírez, J. Vía, I. Santamaría, and L. Scharf, "Detection of spatially correlated time series," IEEE Transactions on Signal Processing, vol. 58 , pp. 5006-5015, Oct. 2010.

[16] D. Ramírez, G. Vazquez-Vilar, R. López-Valcarce, J. Vía, and I. Santamaría, "Detection of rank-p signals in cognitive radio networks with uncalibrated multiple antennas," IEEE Transactions on Signal Processing, vol. 59, pp. 3764-3774, Aug. 2011.

[17] R. López-Valcarce, G. Vazquez-Vilar, and J. Sala, "Multiantenna spectrum sensing for cognitive radio: overcoming noise uncertainty," in Proceedings of the 2010 2nd International Workshop on Cognitive Information Processing, pp. 310-315, 2010.

[18] S. Wilks, "On the independence of $\mathrm{k}$ sets of normally distributed statistical variables," Econometrica, vol. 3, pp. 309-326, Jul. 1935

[19] T. Jiang and F. Yang, "Central limit theorems for classical likelihood ratio tests for high-dimensional normal distributions," The Annals of Statistics, vol. 41, no. 4, pp. 2029-2074, 2013.

[20] T. Jiang and Y. Qi, "Likelihood ratio tests for high-dimensional normal distributions," Scandinavian Journal of Statistics, vol. 42, pp. 988-1009, 2015.

[21] M. Srivastava, "Some tests criteria for the covariance matrix with fewer observations than the dimension," Acta Comment. Univ. Tartu. Math, pp. 77-93, 2006.

[22] Z. Li and J. Yao, "Testing the sphericity of a covariance matrix when the dimension is much larger than the sample size," Electronic Journal of Statistics, vol. 10, no. 2, pp. 2973-3010, 2016.

[23] D. Ramírez, J. Vía, I. Santamaría, and L. Scharf, "Locally most powerful invariant tests for correlation and sphericity of gaussian vectors," IEEE Transacions on Information Theory, vol. 59, no. 4, pp. 2128-2140, 2013.

[24] Z. Bai and J. Silverstein, "CLT for linear spectral statistics of largedimensional sample covariance matrices," The Annals of Probability, vol. 32, no. 1A, pp. 553-605, 2004.

[25] Z. Bai, D. Jiang, J. Yao, and S. Zheng, "Corrections to LRT on large dimensional covariance matrix by RMT," The Annals of Statistics, vol. 37, no. 6B, p. 3822-3840, 2009 
[26] J. Najim and J. Yao, "Gaussian fluctuations for linear spectral statistics of large random covariance matrices," Annals of Applied Probability, vol. 26, no. 3, pp. 1837-1887, 2016.

[27] S. Zheng, Z. Bai, and J. Yao, "Substitution principle for CLT of linear spectral statistics of high-dimensional sample covariance matrices with applications to hypothesis testing," The Annals of Statistics, vol. 43, no. 2 , pp. $546-591,2015$.

[28] J. Gao, G. Pan, and Y. Yang, "High dimensional correlation matrices: Clt and its applications," http://arxiv.org/abs/1411.0081.

[29] Y. Qiu and S. X. Chen, "Test for bandedness of high-dimensional covariance matrices and bandwidth estimation", The Annals of Statistics, vol. 40, no. 3, pp. 1285-1314, 2012.

[30] R. Horn and C. Johnson, Matrix Analysis. Cambridge: Cambridge University Press, 1985

[31] R. Horn and C. Johnson, Topics in matrix analysis. 1991.

[32] Z. Bai and J. Silverstein, "No eigenvalues outside the support of the limiting spectral distribution of large dimensional sample covariance matrices," Annals of probability, vol. 26, pp. 316-345, 1998.

[33] J. Silverstein, "Strong convergence of the empirical distribution of eigenvalues of large dimensional random matrices," Journal of Multivariate Analysis, vol. 5, pp. 331-339, 1995.

[34] V. Girko, "Strong law for the eigenvalues and eigenvectors of empirical covariance matrices," Random Operators and Stochastic Equations, vol. 4, no. 2, pp. 176-204, 1996

[35] X. Mestre, "On the asymptotic behavior of the sample estimates of eigenvalues and eigenvectors of covariance matrices," IEEE Transactions on Signal Processing, vol. 56, pp. 5353-5368, Nov. 2008.

[36] X. Mestre, F. Rubio, and P. Vallet, "Improved estimation of the logarithm of the covariance matrix," in Proceedings of the IEEE Sensor Array and Multichannel Signal Processing Workshop (SAM), (Hoboken (NJ)), pp. 377-380, June 2012.

[37] W. Hachem, O. Khorunzhiy, P. Loubaton, J. Najim, and L. Pastur, "A new approach for mutual information analysis of large dimensional multi-antenna channels," IEEE Transactions on Information Theory, vol. 54, pp. 3987-4004, Sep. 2008.

[38] L. Pastur and M.Shcherbina, Eigenvalue Distribution of Large Random Matrices, vol. 171 of Mathematical Surveys and Monographs. American Mathematical Society, 2011.

[39] W. Hachem, P. Loubaton, X. Mestre, J. Najim, and P. Vallet, "Large information plus noise random matrix models and consistent subspace estimation in large sensor networks," Random Matrices: Theory and Applications, vol. 1, Apr. 2012.

[40] L. Chen, "An inequality for the multivariate normal distribution," Jour nal of Multivariate Analysis, vol. 12, pp. 306-315, 1982.

[41] G. Visick, "A quantitative version of the observation that the Hadamard product is a principal submatrix of the Kronecker product," Linear Algebra and its Applications, vol. 304, pp. 45-68, 2000.

[42] Z. Bai, B. Miao, and G. Pan, "On asymptotics of eigenvectors of large sample covariance matrix," The Annals of Probability, vol. 35, no. 4, pp. 1532-1572, 2007.

\begin{tabular}{|c|}
\hline \\
\\
PLACE \\
PHOTO \\
HERE \\
\end{tabular}

Xavier Mestre (S'96, M'03, SM'09) received the $\mathrm{MSc}$ and $\mathrm{PhD}$ in Electrical Engineering from the Technical University of Catalonia (UPC) in 1997 and 2002, respectively, and the Licentiate Degree in Mathematics in 2011. During the pursuit of his $\mathrm{PhD}$, he was recipient of a 1998-2001 $\mathrm{PhD}$ scholarship (granted by the Catalan Government) and was awarded the 2002 Rosina Ribalta second prize for the best doctoral thesis project within areas of Information Technologies and Communications by the Epson Iberica foundation. From January 1998

to December 2002, he was with UPC's Communications Signal Processing Group, where he worked as a Research Assistant and participated actively in several European-funded projects. In January 2003 he joined the Telecommunications Technological Center of Catalonia (CTTC), where he currently holds a position as a Senior Research Associate and head of the Advanced Signal and Information Processing Department. During this time, he has actively participated in multiple European projects (including the coordination of the EMPhAtiC project, in 2012-15) and in several contracts with the European Space Agency and the local industry. He is IEEE Senior member and elected member of the IEEE Sensor Array and Multi-channel Signal Processing technical committee (2013-present). He has been associate editor of the IEEE Transactions on Signal Processing (2008-11, 2015-present), technical chair of the European Signal Processing Conference EUSIPCO 2011 and general co-chair of the European Wireless Conference 2014
PLACE

PHOTO

HERE
Pascal Vallet (S'08, M'11) received the M.Sc and $\mathrm{Ph} . \mathrm{D}$ degrees respectively from ESIEE Paris (France) in 2008 and Universit Paris-Est (France) in 2011. Since 2012, he has been Associate Professor at Bordeaux-INP (France). His research interests include statistical signal processing for digital communication systems, with a special emphasis on spectral analysis for large dimensional antenna arrays. 